Materials Science and Engineering Laboratory

\title{
REFERENCE
}

NIST

PUBLICATIONS

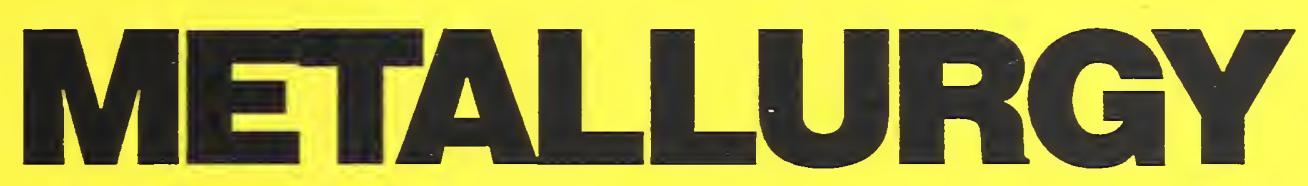

NAS-NRC

Assessment Panel

February 13-14, 1992

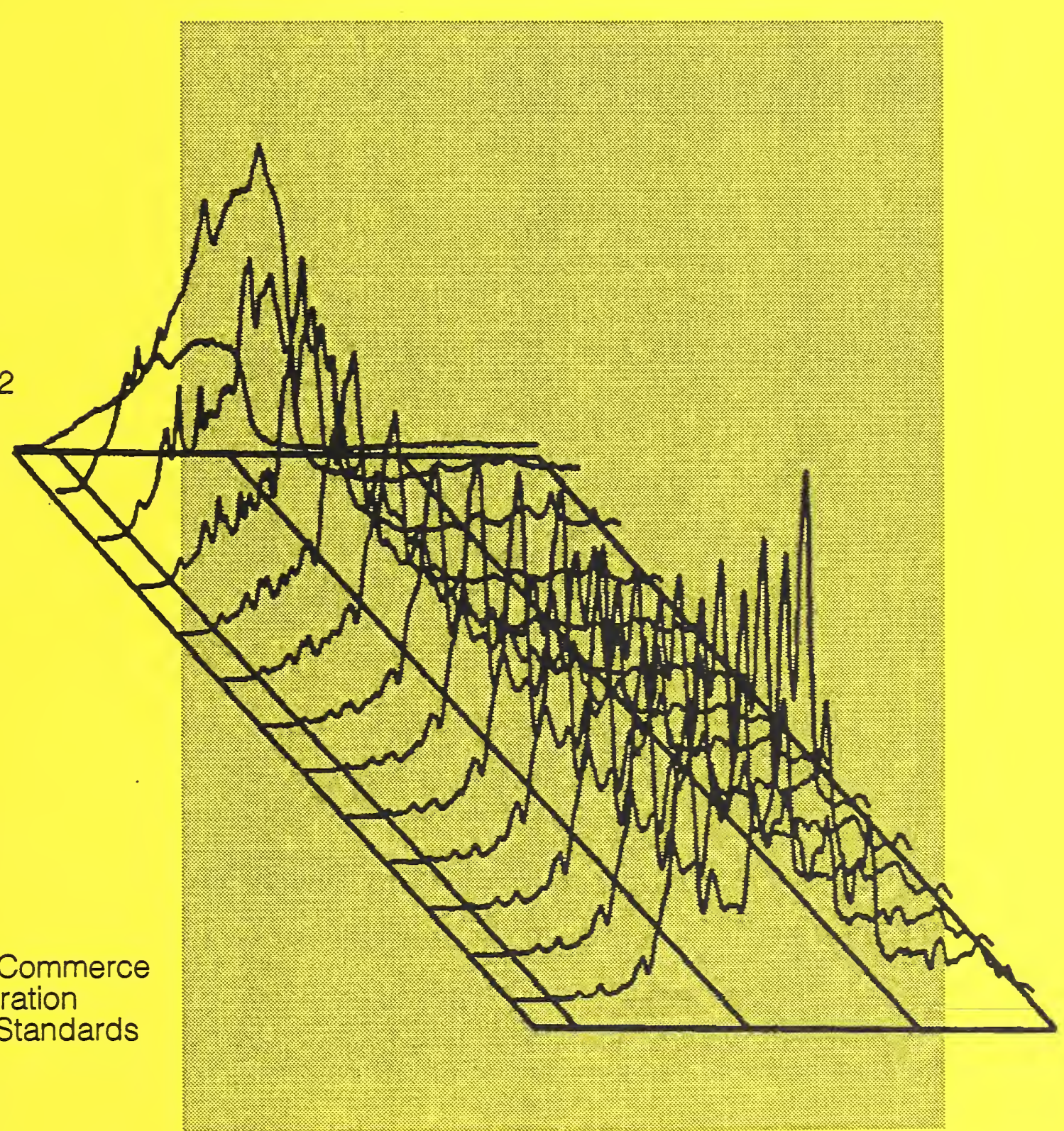

NISTIR 4697

U.S. Department of Commerce Technology Administration

National Institute of Standards and Technology 


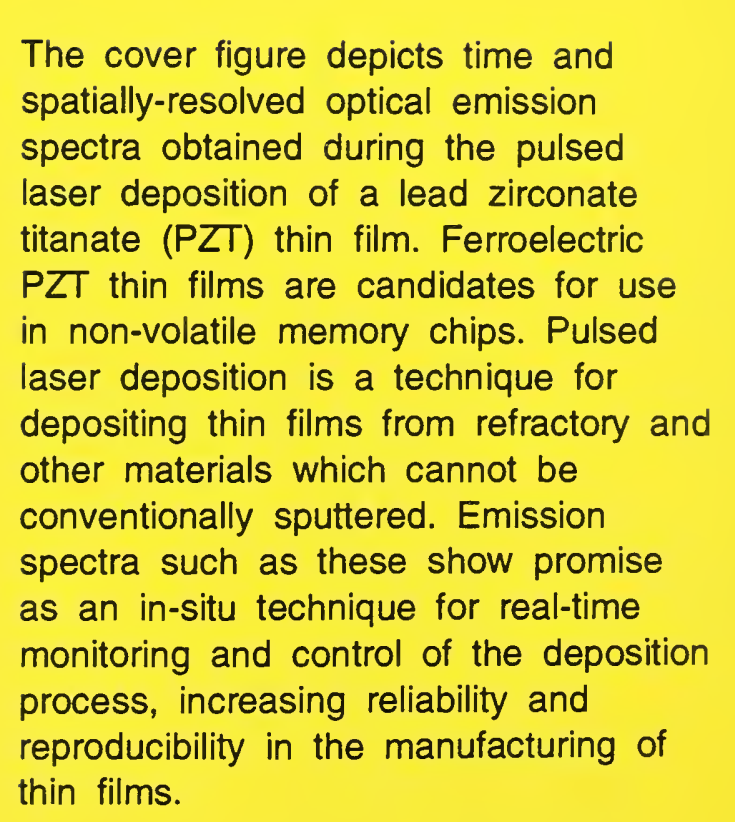


Materials Science and Engineering Laboratory

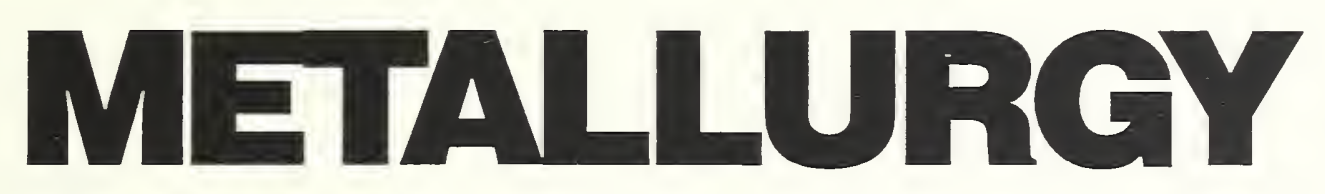

E.N. Pugh, Chief

S.C. Hardy, Deputy

NAS-NRC

Assessment Panel

February 13-14, 1992

NISTIR 4697

U.S. Department of Commerce

Technology Administration

National Institute of Standards

and Technology

\section{Technical Activities 1991}

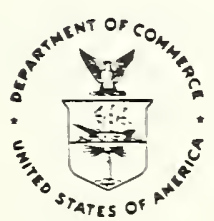

U.S. DEPARTMENT OF COMMERCE, Robert A. Mosbacher, Secretary Technology Administration, Robert M. White, Under Secretary

National Institute of Standards and Technology, John W. Lyons, Director 
Certain companies and commercial products are mentioned in this report. They are used to either completely specify a procedure or describe an interaction with NIST. Such a mention is not meant as an endorsement by NIST or represent the best choice for that purpose. 


\section{METALLURGY DIVISION}

\section{$\underline{\text { CHIEF }}$}

E. Neville Pugh

Phone: (301) 975-5960

\section{DEPUTY CHIEF}

Stephen C. Hardy

Phone: (301) 975-6159

\section{SPECIAL ASSISTANT}

John H. Smith

Phone: (301) 975-5961

\section{GROUP LEADERS}

Corrosion

Richard E. Ricker

Phone: (301) 975-6023

Electrodeposition

David S. Lashmore

Phone: (301) 975-6405

High Temperature Materials Chemistry

John W. Hastie

Phone: (301) 975-5754

Magnetic Materials

Lawrence $\mathrm{H}$. Bennett

Phone: (301) 975-5966

Mechanical Properties and Performance

Leonard Mordfin

Phone: (301) 975-6168

Metallurgical Processing

John R. Manning

Phone: (301) 975-6157

Metallurgical Sensing \& Modeling

Leonard Mordfin (Acting)

Phone: (301) 975-6140

Microscopy Facility

John H. Smith

Phone: (301) 975-5961 

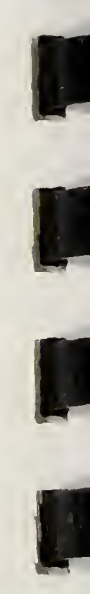

1.

6

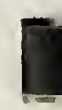

5

5

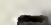




\begin{abstract}
This report summarizes the FY 1991 activities of the Metallurgy Division of the National Institute of Standards and Technology (NIST). These activities center upon the structure-processingproperties relations of metals and alloys, on methods of measurement, and on the generation and evaluation of critical materials data. Efforts comprise studies of metals processing and process sensors; advanced materials - including metal matrix composites, intermetallic alloys and superconductors; corrosion and electrodeposition; mechanical properties; magnetic materials; and high temperature reactions.

The work described also includes cooperative programs with a professional society (the Corrosion Data Program with the National Association of Corrosion Engineers); with a trade association (the Temperature Sensor Program with the Aluminum Association) and several with industry including the Powder Atomization Consortium with two companies.

The scientific publications, committee participation, and other professional interactions of the 74 full-time and part-time permanent members of the Metallurgy Division and its 46 guest researchers are identified.
\end{abstract}




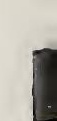

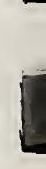

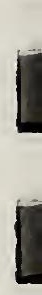

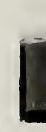

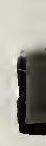

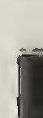

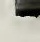

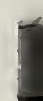

5

5

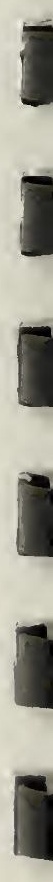




\section{TABLE OF CONTENTS}

Page

OVERVIEW . . . . . . . . . . . . . . . . . . . . . 1

METALLURGY DIVISION

ORGANIZATION CHART . . . . . . . . . . . . . . . . . . . 7

RESEARCH STAFF . . . . . . . . . . . . . . . . . 9

TECHNICAL ACTIVITIES

Corrosion . . . . . . . . . . . . . . . . . . . 15

Electrodeposition . . . . . . . . . . . . . . . . 27

High Temperature Materials Chemistry . . . . . . . . . . . . 43

Magnetic Materials . . . . . . . . . . . . . . . 51

Mechanical Properties and Performance . . . . . . . . . . . . 63

Metallurgical Processing . . . . . . . . . . . . . 73

Metallurgical Sensing \& Modeling . . . . . . . . . . . . . . . . 89

OUTPUTS/INTERACTIONS

Publications . . . . . . . . . . . . . . . . . . 99

Industrial and Academic Interactions . . . . . . . . . . . . . . 115

Technical/Professional Committee

Leadership Activities

APPENDIX

Organizational Chart

National Institute of Standards \& Technology . . . . . . . . . . Al

Organizational Chart

Materials Science and Engineering Laboratory . . . . . . . . . . A2 


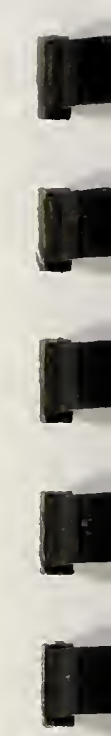




\title{
OVERVIEW
}

\section{METALLURGY DIVISION (855)}

\author{
E. Neville Pugh, Chief \\ Stephen C. Hardy, Deputy Chief \\ June Toms, Secretary
}

The Division has continued to consolidate its thrust in materials processing, particularly in the areas of process sensing and process modeling. This activity, aimed primarily at the intelligent processing of materials (IPM) and other forms of automated process control, is fully consistent with NIST's traditional measurement science mission and is particularly appropriate to NIST's mandate to assist US industry in improving competitiveness. The transfer of the results of these programs to industry is being accomplished by a consortium (on powder processing), expanded in 1991, and through Cooperative Research and Development Agreements (CRDAs) with specific companies. The Division has maintained strong programs in the traditional NIST areas of materials characterization, data and Standard Reference Materials (SRMs). In the former, the focus is on advanced materials, including metal matrix composites, intermetallic alloys, nanostructured magnetic materials, and high $\mathrm{T}_{c}$ superconductors, but a major program alos is being conducted on $\mathrm{Pb}-\mathrm{Sn}$ solder, a material which has been widely used in the electronic industries for many years, yet, surprisingly, is poorly understood. The NACE-NIST Corrosion Data Program, now the major data activity in the Division, has continued to develop successful collaborative programs with industry to develop computerized databases and expert systems for use in materials selection and corrosion control. An exciting new activity in the standards area, the establishment of a traceable national hardness standard at NIST, has proceeded on schedule.

Highlights of the Division's programs, described in more detail in the reports of the individual groups, are conveniently summarized under three categories:

\section{Materials Processing}

Powder processing continues to be a major thrust, with programs involving both powder atomization and consolidation. The former centers on the Metallurgical Processing Group's high pressure inert gas atomizer, and a consortium with several companies and two other NIST Laboratories (Chemical Science and Technology, and Manufacturing Engineering). In 1991, the consortium began a second three-year phase, with the number of industrial partners increasing to four (Crucible Materials Corp., General Electric Co., Martin Marietta Energy Systems, Inc., and Pratt and Whitney Aircraft) and with the Department of Energy becoming a member. The program continues to be directed toward the measurement and real-time control of powder size during atomization of engineering alloys (the present focus is on a $\mathrm{Ni}$-based superalloy) and builds on the particle-size sensor and expert system based process controller developed in the first phase. The sensor combines Fraunhofer diffraction optics and pattern recognition software, and modifications to the latter over the past year have improved the accuracy of particle size analysis by several 
orders of magnitude compared to present iterative techniques and also permit the resolution of more complex size distributions. Advances have been made in studies of the atomization process, using both experimental and theoretical approaches. Significantly, computer codes being used on the Cray Y-MP at NIST for modeling supersonic gas flow have been modified for PC use by consortium members.

Studies of powder consolidation continued to be concentrated on hot isostatic pressing (HIP). During the year, the Metallurgical Processing Group successfully completed its work on a DARPA-supported program on the application of IPM techniques to the consolidation of powders of titanium and titanium-aluminide intermetallics. In this program, which involved BDM International and an industrial consortium, the Group had previously developed and utilized an eddy current sensor for monitoring densification and, in 1991, elucidated the effects of plastic strain introduced by phase transformations on the consolidation process. In a separate study, the HIP technique was used to produce bulk samples of the intermetallic phases such as $\mathrm{Cu}_{6} \mathrm{Sn}_{5}$ which form in solder joints. This activity played an important part in a major program on solderability sponsored by the U.S. Army Harry Diamond Laboratory.

The Metallurgical Sensing and Modeling Group (formerly the Advanced Sensing Group) has continued its development of eddy-current and ultrasonic sensors. The versatility of the former is becoming apparent. Used previously in HIP studies and to measure internal temperatures in $\mathrm{Al}$ alloys during extrusion, the eddy-current technique was extended in 1991 to the rolling of Al alloy sheet (with the Aluminum Association) and to the pyrolysis of carbon-carbon composites during high temperature processing (with General Dynamics Corp., and DARPA). In addition, programs were initiated to apply the technique to the measurement of case depths on steel automotive shafts (under a CRDA with General Motors Corp.), and to the determination of liquid metal flow rate through the delivery tube in powder atomization (as part of the NIST Atomization Consortium). Progress has also been made in the theory underlying the technique. Thus a general theory has been developed for eddy current inversion, which permits measurement of the layer thicknesses in layered materials and has potential for evaluating the shape of discrete flaws. Work on the ultrasonic technique remains focused on interfaces. In the on-going program on sensing of the liquid/solid interface in metals, the significant scattering of ultrasonic waves by grain boundaries in the solid has led to the use of waves transmitted in the liquid rather than those transmitted through the solid metal. Promising results have been obtained using lasers to generate and receive the reflected ultrasonic waves, and it is hoped that the method ultimately will yield information on microstructural features of the interface as well as on its position. In another interesting development, the interface between solid materials is being studied by launching ultrasonic waves along the interface and imaging the acoustic energy radiated from each point on the interface at the outer surface using an acoustic microscope.

The Division maintained a strong effort in process modeling during 1991 . In addition to the on-going activities in powder atomization and HIP, the Metallurgical Processing Group's modeling in the solidification area was broadened. Thus the NASA supported program on gravity related effects on interface stability was extended to an investigation of laser processing of 
silicon wafers, an important technique in the semiconductor industry; the results permit changes in interface morphology (planar, cellular or dendritic) to be correlated with increases in laser power. The Group, which works closely with the Computing and Applied Mathematics Laboratory in this area, has also developed a new modeling approach which predicts the growth pattern of the solid phase during alloy solidification and, in particular, yields the segregation pattern in the final solid products. The Metallurgical Sensing and Modeling Group has continued its major DARPA supported program on modeling of microstructure evolution in intermetallic alloys. A highlight of this year was a three-week workshop which brought together materials scientists, physicists and mathematicians to address the problem. Experiments to evaluate the theoretical models also were initiated.

Electrodeposition has great potential for the synthesis and processing of advanced materials, and the Electrodeposition Group has become an acknowledged leader in this effort. During the year, the Group continued its pioneering work on the electrodeposition of nanoscale layered structures, producing multilayered materials of $\mathrm{Co}-\mathrm{Cu}, \mathrm{Co}-\mathrm{Cr}$ and $\mathrm{Cu}-\mathrm{Ni}$. There is considerable commercial interest in the magnetic and mechanical properties of these materials, and these are being studied in the Magnetic Materials (with support from IBM) and the Mechanical Properties and Performance (supported by the U.S. Air Force Wright Laboratory) Groups, respectively. Another innovative program involves the electrodeposition of titanium aluminides and other Ti-Al alloys onto continuous ceramic fibers, e.g., SiC, as the first step in the fabrication of composites of these materials. Significant progress was made in a program funded by the Office of Naval Research and DARPA to develop a continuous fiber plating line for this purpose; the Group is well positioned to attack this problem because of its previous modeling studies of this process carried out under Competence Funding.

Another commercially important method of producing thin layers is vapor deposition, and this is the emphasis of work in the High Temperature Materials Chemistry Group. During the year, their laser deposition facility, upgraded by the installation of an excimer laser system, was used to produce both electronic (PZT) and magnetic $\left(\mathrm{Fe}_{3} \mathrm{O}_{4}-\mathrm{Ag}\right.$ nanocomposites) thin films. The thrust of these programs is to characterize the species present in the vapor phase using our unique mass and optical spectroscopy facilities, and hence to develop basic understanding of the deposition processes. Such knowledge is considered to be essential for the efficient control of commercial vapor deposition processes.

\section{Materials Characterization}

Work on intermetallic alloys has emerged as a major thrust in the Division's characterization programs, generally involving collaboration between several groups. Because of their great potential as high temperature structural materials for aerospace applications, the titanium aluminides are attracting much of our effort. It was seen in the Materials Processing section that a major program is underway in the Electrodeposition Group to synthesize these materials electrochemically by deposition onto fibers. In parallel studies, the structure of the electrodeposited layers is being investigated. To date, the effort has been on the $\mathrm{Al}_{3} \mathrm{Ti}$ intermetallic formed from chloroaluminate 
electrolytes, and has shown that extensive (111) twinning exists, the twins possibly acting as a source of the ledges necessary for crystal growth. In the Metallurgical Processing Group, DARPA supported research has continued on $\mathrm{Ti}-\mathrm{Al}-\mathrm{Nb}$ intermetallic alloys and several significant accomplishments have resulted. For example, patent application has been filed for a series of the ternary alloys designed to have low density, room temperature ductility, and high temperature strength and stability; the high temperature properties result from the formation of a composite of two phases, a relatively ductile orthorhombic phase and a plastically unshearable omega-type intermetallic, which are in equilibrium up to $800{ }^{\circ} \mathrm{C}$. These and other studies are supported by a range of modeling activities designed to generate calculated phase diagrams.

Other intermetallic compounds are being studied in the Corrosion Group. This work is being carried out on both nickel and iron aluminides produced at the Oak Ridge National Laboratory. The commercialization of these alloys as substitutes for more expensive $\mathrm{Ni}$-based alloys and stainless steels is being led by ORNL, with the more sophisticated characterization of behavior in aqueous media being conducted at NIST. Work to date has established that the active-passive behavior of $\mathrm{Ni}_{3} \mathrm{Al}$ is essentially the same as that of pure $\mathrm{Ni}$, and that, while embrittlement can result from cathodically generated hydrogen, this is unlikely in environments encountered in normal service. These results, encouraging to potential users, were presented at a recent technology transfer meeting at ORNL attended by over thirty representatives of various companies.

A continuing program on the characterization of solder joints also centered on intermetallic alloys. This activity, supported by the U.S. Army Harry Diamond Laboratory, deals with the influence of intermetallic phases on the performance of solder joints used in the microelectronics industry. Since $\mathrm{Pb}-$ Sn solder on $\mathrm{Cu}$ substrates remains the predominant couple, effort has focused on the $\mathrm{Cu}_{6} \mathrm{Sn}_{5}$ and $\mathrm{Cu}_{3} \mathrm{Sn}$ phases which form at the junction. The Metallurgical Processing Group prepared bulk samples of these compounds and working with the Mechanical Properties and Performance Group have generated data on a broad range of physical and mechanical properties of these materials; such data are essential for the modeling studies of solder joint behavior being conducted in the Division and at other laboratories. In addition, the wetting of the intermetallics by the $\mathrm{Pb}-\mathrm{Sn}$ solder, thought to be critical in the soldering of pre-tinned $\mathrm{Cu}$ components, has been investigated by spreading-area and wettingbalance tests. Results to date suggest that air-formed oxide films on the intermetallics play an important role in the wetting process. Pre-tinning is commonly carried out by electrodeposition, and the Electrodeposition Group has studied this approach, depositing layers of both solder and the intermetallic alloys, as well as thin barrier layers to prevent reaction between the solder and $\mathrm{Cu}$ substrate.

Metal matrix composites (MMC) represent an important emerging technology in which we have maintained a significant effort, both in continuous fiber reinforced materials for high performance applications and, beginning in 1991 , discontinuously reinforced composites which are more likely to penetrate the automotive and other large commercial markets. Work on the former deals mainly with titanium aluminides reinforced with $\mathrm{SiC}$ or $\mathrm{Al}_{2} \mathrm{O}_{3}$ fibers, although 
some studies over the past year involved Ni-graphite composites; interface stability at elevated temperatures continues to be studied in the

Metallurgical Processing and Electrodeposition Groups for both materials. Work on discontinuously reinforced MMCs has been initiated in the Mechanical Properties and Performance Group in collaboration with several MMC producers. So far, the program has been concerned with Al MMCs of potential interest to the automotive industry and has addressed creep and fatigue at elevated temperatures, two processes of critical importance to engine applications. Two key questions are being considered: (1) how applicable are present standard test methods, and (2) what are the microstructural bases for creep and fatigue resistance?

The Magnetic Materials Group has maintained strong programs on nanocomposite materials, specifically on mixtures of nanoscale metals and oxides. These materials have been shown to have unusual composition-dependent magnetic properties, and thus they hold promise of atomic engineering of specific properties. During the year, studies have continued on thin films of $\mathrm{Ag}-\mathrm{Fe}_{3} \mathrm{O}_{4}$ produced by vapor deposition techniques, bulk Fe-vitreous alumina prepared by the sol-gel process, and bulk $\mathrm{Cu}-\mathrm{Fe}$ and $\mathrm{Cu}-\mathrm{Co}$ formed by coprecipitation from aqueous solutions. In all cases, the magnetic properties have been found to be dependent on processing variables, and these effects are being correlated with the structure of the material and the chemical state of the constituents, determined by a variety of techniques including Mossbauer spectroscopy, $x$-ray diffraction and electron microscopy. The recognition, in 1990, that nanocomposites may possess giant magneto-caloric effects, and hence great potential for magnetic refrigeration, has been pursued in 1991 along both theoretical and experimental paths. The former confirms the feasibility of the approach, and equipment to obtain definitive experimental proof is being assembled. Interestingly, the enhanced effect in nanocomposite material is expected at higher temperatures than those presently employed in magnetic refrigerators, and so this technology is potentially feasible for use in automotive air conditioning systems; a CRDA has been signed with General Motors Corporation to pursue this possibility.

Among other programs on characterization, that on high temperature superconductors is particularly worthy of note. A key to these studies is the availability of high quality YBCO mono- and bicrystals prepared by the Metallurgical Processing Group (in collaboration with the Ceramics Division). Using twinned and de-twinned monocrystals, the Magnetic Materials Group demonstrated that flux pinning due to twin boundaries, previously thought to be small, is strongly dependent on temperature and the magnitude and orientation of the applied field. In particular, no effect of twin boundaries on pinning was observed at any temperature for applied fields perpendicular to the twin boundaries, whereas significant pinning was found over certain temperature ranges when the field was parallel to the boundaries. In the Metallurgical Processing Group, a systematic study of bicrystals is in progress to examine effects of grain boundaries, known to be "weak links" in electrical transport in normal polycrystalline material. The program at NIST is focussed on characterizing grain boundary structures, using the new phenomenon of twin pattern matching, while transport measurements are being made at the University of Wisconsin. 
The Corrosion Data Program, formed in cooperation with the National Association of Corrosion Engineers (NACE) to respond to a critical need for a centralized, computerized database of corrosion data, continues to be the Division's major data activity. Industrial support for focused, knowledgebased expert systems remained strong in 1991, with the Materials Technology Institute of the Chemical Process Industries, the Electric Power Research Institute, and the International Copper Association each sponsoring specific projects. During the year, four PC-based systems were released for public distribution, bringing the total to ten, and nine more were distributed for industrial review prior to public release.

In the standards area, the new program to establish a traceable national hardness standard at NIST proceeded on schedule. This program grew out of an earlier comparison of test blocks used by block manufacturers to calibrate Rockwell hardness test machines. The comparison, carried out by the Mechanical Properties and Performance Group in collaboration with ASTM and published in February 1991, indicated that US test blocks are not compatible with the presently used international scale, and led to the decision to develop a national hardness standard traceable to NIST. This standard is to be based on direct measurements of force, displacement of the indentor and time, quantities which are traceable to NIST basic standards, rather than on comparison with existing blocks. During 1991, construction was begun on a precision dead weight machine, similar in design to that at the Italian National Measurement Laboratory (IMGC) in Turin on which European hardness standards are currently based. Construction of the basic machine is being carried out in Italy and should be completed in 1992.

The Mechanical Properties and Performance Group also became involved in another important national issue in 1991, namely the accreditation of laboratories for inspecting and testing fasteners. This stemmed from the recognition that millions of fasteners sold in the US are mismarked, substandard, counterfeit, or nonconforming in some manner, and from the resulting Fastener Quality Act. The Act requires that NIST's National Voluntary Laboratory Accreditation Program (NVLAP) develop the procedures to be used to accredit testing laboratories and the Division is assisting NVLAP in this activity. 

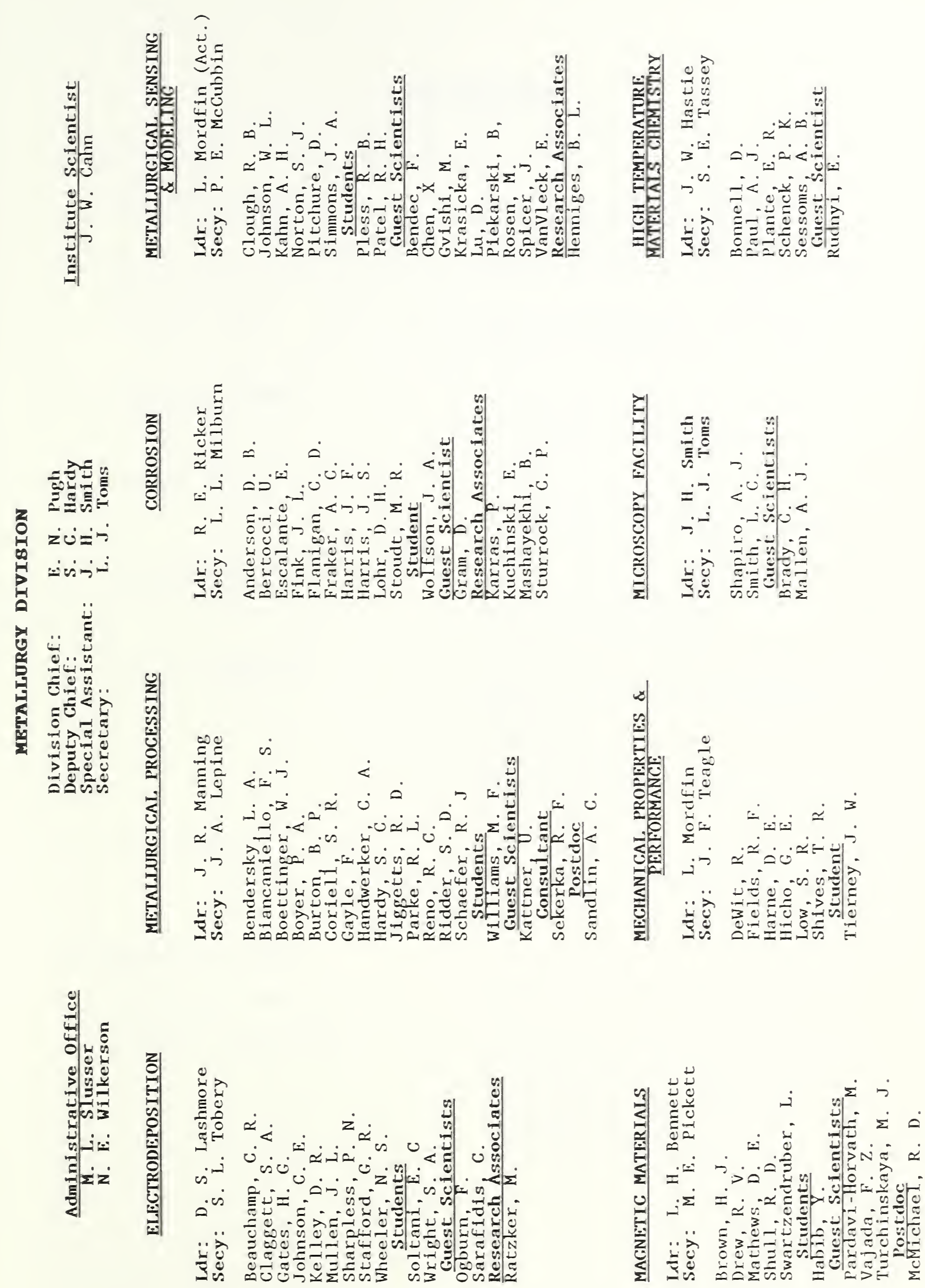


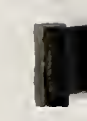




\section{RESEARCH STAFF}

\section{Corrosion Group}

Anderson, David B.

Bertocci, Ugo

Escalante, Edward

Fraker, Anna C.

Harris, Jonice S.

Ricker, Richard E.

Stoudt, Mark R.

\section{Eletrodeposition Group}

Beauchamp, Carlos R.

Johnson, Christian E.
- Industrial corrosion testing
- Corrosion data evaluation
- Corrosion database development
- Expert systems for corrosion control

- Electrochemical measurements

- Computer modeling

- Passivity and pitting

- Underground corrosion

- Corrosion in concrete

- Corrosion rate measurements

- Titanium alloys

- Corrosion processes

- Transmission electron microscopy

- Surgical implant metals

- Scanning electron microscopy

- Corrosion measurements

- Mechanical properties

- Environmental induced fracture (stress corrosion cracking and corrosion fatigue)

- Hydrogen embrittlement

- Aluminum alloys

- Advanced materials (composites and intermetallics)

- Advanced materials

- Environmentally induced fracture

- Corrosion engineering 
Kelley, David R.

Lashmore, David S.

Mullen, Jasper L.

Soltani, Elaine C.

Stafford, Gery R.

Wheeler, Nea S.
- Microhardness SRM development

- Dye penetrant SRM development

- Precious metal electrodeposition

- Plating on aluminum

- Electrochemical mechanisms of coating processes

- Pulsed alloy deposition

- Composition modulated alloy deposition

- Properties and structure of electrodeposited coatings

- Amorphous alloys

- Transmission electron microscopy

- Metal matrix composites

- Development of automated hardness testing

- Electrochemical measurements for determining metal corrosion

- Analytical spectroscopy

- Scanning electron microscopy

- Composition modulated alloys

- Electrochemical transients

- Electrodeposition

- Molten salt electrochemistry

- Alloy deposition

- Scanning electron microscopy

- Transmission electron microscopy

\section{High Temperature Materials Chemistry Group}

Bonne11, David W.

Hastie, John W.
- High temperature-pressure mass spectrometry

- Computer modeling

- Levitation calorimetry

- Laser vaporization mass spectrometry

- High temperature chemistry of inorganic materials

- Phase equilibria thermochemistry and solution models

- High temperature-pressure mass spectrometry

- Chemistry of combustion 


$\begin{array}{ll}\text { Paul, Albert J. } & \circ \text { Laser Physics } \\ & \circ \text { Plasma Diagnostics } \\ & \circ \text { Spectroscopy } \\ & \circ \text { Thin Film Deposition } \\ & \\ \text { Plante, Ernest R. } & \circ \text { Thermodynamics } \\ & \circ \text { Mass spectrometry } \\ \text { Schenck, Peter K. } & \circ \text { Laser spectroscopy } \\ & \circ \text { Emission Spectroscopy } \\ & \circ \text { Thin film deposition } \\ & \circ \text { Computer graphics }\end{array}$

\section{Magnetic Materials Group}

\begin{tabular}{|c|c|c|}
\hline Bennett, Lawrence & $\begin{array}{l}0 \\
0 \\
0 \\
0 \\
0\end{array}$ & $\begin{array}{l}\text { Magnetic materials } \\
\text { Magnetocaloric effect } \\
\text { High } \mathrm{T}_{c} \text { superconductors } \\
\text { Topology of local environments } \\
\text { Alloy phase stability }\end{array}$ \\
\hline McMichael, Robert D. & $\begin{array}{l}\circ \\
\circ \\
\circ \\
\circ\end{array}$ & $\begin{array}{l}\text { Ferromagnetic resonance } \\
\text { Magnetic Anisotropy } \\
\text { Nanocomposites } \\
\text { Magnetocaloric effect }\end{array}$ \\
\hline Brown, Henrietta $\mathrm{J}$. & $\begin{array}{l}\circ \\
\circ\end{array}$ & $\begin{array}{l}\text { High } T_{c} \text { Superconductors } \\
\text { Magnetization measurements }\end{array}$ \\
\hline Shull, Robert D. & $\begin{array}{l}0 \\
0 \\
0 \\
0 \\
0\end{array}$ & $\begin{array}{l}\text { Nanocomposites } \\
\text { Magnetic susceptibility } \\
\text { Mössbauer effect } \\
\text { X-ray and neutron diffraction } \\
\text { Magneto-caloric and Magneto-optical } \\
\text { effects }\end{array}$ \\
\hline Swartzendruber, Lydon $\mathrm{J}$. & $\begin{array}{l}0 \\
0 \\
0 \\
0\end{array}$ & $\begin{array}{l}\text { Magnetic susceptibility } \\
\text { Magnetic methods, NDE } \\
\text { Gamma-ray resonance spectroscopy } \\
\text { Barkhausen effect }\end{array}$ \\
\hline
\end{tabular}

\section{Mechanical Properties and Performance Group}

deWit, Roland

- Fracture mechanics

- Dislocation theory

- Stereology

Fields, Richard J.

- Mechanical properties

- High temperature materials

- Quantitative metallography 
Hicho, George E.

Low, Samuel R., III

Mordfin, Leonard

Shives, T. Robert

Biancaniello, Francis S.

Boettinger, William J .

Burton, Benjamin P.

Coriell, Sam R.
- Mechanical properties

- Ferrous metallurgy

- Failure analysis

- Fracture toughness

- Mechanical properties and testing

- Laboratory computer systems programming

- Automated test design

- Mechanical test methods

- Materials engineering

- Stress and structural analysis

- Mechanical properties and testing

- Hardness test methods

- Failure analysis

- Analytical transmission electron microscopy

- Aluminum alloys; quasicrystals

- Intermetallics for high temperature application

- Inert gas atomization; metal powder processing and consolidation

- Special alloys, composites, heat treating, and quasicrystal preparation.

- Melt-spinning; rapid solidification

- Relation of alloy microstructures to processing conditions

- High temperature alloys/intermetallics

- Rapid solidification

- First principles phase diagram calculations

- Phase equilibria experiments

- Theoretical studies of order-disorder phenomena in alloys

- Modeling of solidification processes

- Interface stability

- Convection and alloy segregation during solidification 
Gayle, Frank W.

Handwerker, Carol A.

Hardy, Stephen C.

Jiggetts, Rodney D.

Kattner, Ursula R.

Manning, John R.

Ridder, Stephen D.

Schaefer, Robert J.

Williams, Maureen E.
- Aluminum metallurgy

- Transmission electron microscopy

- Structure/property relationships

- High $T_{c}$ superconductors

- Interface studies; boundary migration

- Metal matrix composites

- Solderability

- Alloy coarsening

- Surface tension measurements

- Interface segregation

- Hot isostatic pressing

- Quantitative metallography

- Solder joint intermetallics

- Phase diagram evaluations

- Composites

- Intermetallics

- Metals processing

- Diffusion kinetics

- Interface reactions

- Inert gas atomization; powder processing

- Microparticle rapid solidification

- Solidification dynamics

- Hot isostatic pressing of intermetallics

- Solidification and solid-state phase transformation processes

- Electron beam rapid solidification

- Differential thermal analysis

- Powder x-ray diffraction

- Solder wettability

\section{Metallurgical Sensing and Modeling Group}

Clough, Roger B.

Kahn, Arnold H.
- Micromechanical Modeling

- Acoustic Emission

- Mechanical properties

- Eddy current modeling

- Electromagnetic theory in NDE

- Solid state physics 
Johnson, Ward L.

Norton, Stephen J.

Pitchure, David J.

Simmons, John A.

Yolken, H. Thomas
- Ultrasonics

- Ultrasonic, eddy current profiling

- Inverse modeling

- Digital electronics

- Ultrasonic instruments

- High temperature measurements

- Modeling of microstructure evolution

- Ultrasonic interface characterization

- Defects and internal stress

- Acoustic emission

- Intelligent processing of materials

- Nondestructive evaluation 
Corrosion reduces the reliability of engineered systems and can cause catastrophic failures involving loss of life and property. Corrosion adversely affects industrial competitiveness by reducing plant efficiencies and by reducing the usable lifetime or quality of industrial products. Battelle Columbus Laboratories and NBS (NIST) conducted a study of the economic effect of corrosion in the United States and concluded that for the year, 1975, corrosion cost the U.S. economy 4.2 percent of the gross national product which is over $\$ 200$ billion per year if one extrapolates this figure to the present. This study also concluded that 15 percent of this cost of corrosion was "avoidable" and could be made available for more productive economic activities if presently available technology were utilized effectively. The remaining costs required technological advances before they could be reduced significantly. The key factors in reducing these losses are (1) better utilization of existing knowledge, (2) the development of a better understanding of corrosion mechanisms, (3) the development of better corrosion measurement science and (4) the development of new corrosion resistant advanced materials.

To promote the better utilization of existing knowledge, the Corrosion Group and the National Association of Corrosion Engineers (NACE) have joined together to create the NACE-NIST Corrosion Data Program. The purpose of this activity is to help industry and government agencies reduce corrosion losses by providing information on materials reliability and corrosion control in a convenient and usable form. The Corrosion Data Program utilizes the latest developments in personal computer information science and collaborates with industries and industrial consortia to develop expert systems and databases that assist designers and corrosion control decision makers. The products of this program are distributed to the public by NACE.

To improve our understanding of corrosion mechanisms, the Corrosion Group is conducting studies into the mechanisms of stress corrosion cracking (SCC), localized corrosion and uniform corrosion. During the past year, the Corrosion Group conducted studies on the influence of stress on the corrosion and stress corrosion cracking of face centered cubic metals and alloys and on the role of grain boundary precipitates in the intergranular stress corrosion cracking of Al-Li alloys. In addition to this work on metallic corrosion, the Corrosion Group conducted an investigation of the hypothesis that exposure to high pressure water would result in a permanent degradation in the mechanical properties of polymer matrix composites.

To promote the development of better corrosion measurement science, the Corrosion Group is developing computer models based on electrochemical reaction kinetics that simulate electrochemical experiments. Last year, the Corrosion Group developed a method for modeling simple anodic reactions that accounts for the influence of complex interactions of ions in the solution (e.g. Cu in aq. ammonia). During FY 91, a model for the interactions between the reactants and products of the two most common cathodic processes that occur during corrosion, oxygen reduction and hydrogen evolution, was developed. The Corrosion Group is investigating the application of these 
models to understanding the results of electrochemical measurements of corrosion behavior and the application of these results to the prediction of usable lifetimes.

To assist the development of new advanced materials, the Corrosion Group is studying the corrosion and stress corrosion cracking behavior of metal matrix composites (Al alloy 8090 with SiC particulate), intermetallic compounds $\left(\mathrm{Ni}_{3} \mathrm{Al}, \mathrm{FeAl}\right.$ and $\mathrm{Fe}_{3} \mathrm{Al}$ ), high strength low-alloy (HSLA) steel, and $\mathrm{Al}-\mathrm{Li}$ alloys.

\section{FY 91 Significant Accomplishments}

- The NACE-NIST Corrosion Data Program continued to collaborate with industry and produce personal computer based corrosion information and advisory systems. During FY 91, 4 systems were released for public distribution and 9 more systems were distributed to industrial review committees for evaluation prior to public release. This brings the total number of systems developed by this joint activity and in public distribution to ten.

- The Corrosion Group designed a simple experiment to test the hypothesis that a crack in a brittle film on the surface of a ductile substrate will propagate into that substrate for some small though finite distance before arresting. This hypothesis is a critical part of the postulated film-induced cleavage mechanism for stress corrosion cracking. The results confirmed this hypothesis and will have a significant impact on our understanding of the mechanism of stress-corrosion cracking as well as other fracture phenomena.

- The Corrosion Group demonstrated that exposure of graphite and glass fiber epoxy matrix composites to high pressure water did not result in a permanent degradation in the mechanical properties as postulated by earlier investigators based on less thorough experimental results.

- The Corrosion Group developed a model for calculating the potentiodynamic polarization behavior and the impedance of a corroding electrode with two simultaneous cathodic reactions: oxygen reduction and hydrogen evolution.

- The Corrosion Group demonstrated that the susceptibility of the parent metal and the heat affected zone of HSLA steel to hydrogen embrittlement is significantly less than that of another steel of similar strength level that is currently in use.

- The Corrosion Group completed the development of a database system for the tracking and analysis of scientific research data pertinent to the storage of high level nuclear waste in a repository and delivered this system to the Nuclear Regulatory Commission and the Center for Nuclear Waste Regulatory Analysis. 
The Corrosion Group presented its work on the corrosion and stress corrosion cracking behavior of nickel and iron aluminide to over thirty representatives of various industries at a technology transfer conference and workshop on nickel and iron aluminides sponsored by Oak Ridge National Laboratory.

\section{Corrosion Data Program}

D. B. Anderson, C. D. Flanigan, P. Karras*, E. J. Kuchinski*, B. Mashayekhi*,

C. P. Sturrock ${ }^{\star}$, and Z. Qu ${ }^{\star \star}$, J.A. Wolfson ${ }^{\star \star \star}$

* NACE Research Associate

** Guest scientist sponsored by the United Nations Industrial Development Organization and Beijing University of Science and Technology.

$* * *$ Student Trainee

The Corrosion Data Program was established in 1982 as a joint program between the NIST Corrosion Group and the National Association of Corrosion Engineers (NACE). The objective of this program is to develop personal computer programs which aid designers and corrosion control decision makers by providing reliable information on materials reliability and corrosion control for engineering materials exposed to different industrial environments.

Activities in FY 91 centered on:

(1) The compilation and validation of data supplied by different industrial sources.

(2) Maintaining a leadership role in the development of consensus standards for corrosion data formats through ASTM and NACE.

(3) The development of materials advisory systems which guide material selection for storage and handling of hazardous chemicals. Four systems were released for public distribution during this FY and five more are in review by committees of industrial experts (sponsored by the Materials Technology Institute of the Chemical Process Industries).

(4) The development of computer systems to guide electric utilities in such diverse areas as corrosion control in condenser systems, environmentally induced fracture, intergranular corrosion in steam generators, corrosion of critical components in flue gas desulfurization systems, microbiological influenced corrosion and corrosion in service water systems. The prototypes of four systems were submitted to industrial review committees during this FY (sponsored by the Electric Power Research Institute).

(5) The development of an expert system on corrosion control in potable water distribution systems (sponsored by the INCRA Technology Division of the International Copper Association). 
Supporting a cooperative program to develop a personal computer program that computes electrochemical equilibrium diagrams from thermodynamic data. The program utilizes the NIST thermodynamic database.

The Corrosion Data Center continues to evolve as a focal point for addressing complex issues in the collection and dissemination of reliable corrosion data using the latest advances in personal computer information science. The Center receives substantial industry financial support and technical input for defining program scope and in assuring consensus on the relevance and applicability of the data.

\section{Corrosion Mechanisms and Corrosion Measurement Science}

U. Bertocci, J. L. Fink, F. Friedersdorf*, J. S. Harris, E. N. Pugh, R. E. Ricker, K. Sieradzki and M. R. Stoudt

* Guest Scientist - Johns Hopkins University

Film-Induced Cleavage Hypothesis - One of the mechanisms proposed to explain SCC is the film-induced cleavage mechanism. One of the key features of this postulated mechanism is the hypothesis that brittle crack which has nucleated in a brittle surface film will continue to propagate in a brittle manner for a short distance when it enters the underlying ductile substrate. To test this hypothesis, the Corrosion Group plated pure nickel with rhodium. Rhodium is a face-centered cubic metal which is brittle and exhibits complete solid solubility in nickel. When these samples were deformed by mechanical loading either in air or in the scanning electron microscope, cracks formed in the rhodium layer and opened up rapidly as the cracks arrested. Figure 1 is a scanning electron micrograph of a cross-section through one of the cracks that formed during bending. In this figure, the rhodium layer is clearly visible as a result of atomic number contrast and it appears that the crack propagated 1 to $1.5 \mu \mathrm{m}$ into the nickel before arresting and blunting. For comparison, figure 2 shows a crack that appears to have arrested at the $\mathrm{Ni}-\mathrm{Rh}$ interface. While considerable work will be required before these observations are fully understood, these results support the hypothesis that brittle films can induce cleavage propagation for short distances in ductile metals which is a key feature of the film-induced cleavage mechanism for SCC.

Mechanism(s) of Stress Corrosion Cracking - While the results above demonstrate that under certain conditions it is possible for a brittle crack in a surface film to propagate into a ductile substrate for a short distance before arresting, it still has to be demonstrated that stress corrosion cracks propagate by this process. To do this, the Corrosion Group has been working with Johns Hopkins University to measure the rate of instantaneous crack propagation during intergranular and transgranular SCC in Ag-Au alloys under different electrochemical conditions. The results to date have shown that the crack velocities are in excess of 3 and $0.3 \mathrm{~mm} / \mathrm{s}$ for transgranular and intergranular cracking respectively. However, these experiments are in progress and better velocity estimates are expected as better experimental techniques are developed. 
Intergranular Stress Corrosion Cracking of Al-Li Alloys - The role of grain boundary precipitates in the intergranular SCC of Al-Ii and Al-Li-Cu alloys was evaluated by conducting slow strain rate tensile tests on samples heat treated to contain different grain boundary precipitate size distributions with the same matrix yield strength and slip character. The results of these tests are shown in figure 3 which is a plot of the environment independent or inert environment value of the strain to failure vs. the strain to failure observed in the environment. In this figure, it can be seen that both the precipitate size distribution and the type of precipitate influence the SCC behavior ( $\mathrm{AlLi}$ in the $\mathrm{Al}-\mathrm{Ii}$ alloy and $\mathrm{Al}_{6} \mathrm{Li}{ }_{3} \mathrm{Cu}$ in the $\mathrm{Al}-\mathrm{Ii}-\mathrm{Cu}$ alloy). Currently, investigations of the influence of strain rate on the crack propagation rate of these alloys is underway.

Computer Modeling of Electrochemical Measurements - The Corrosion Group has been developing computer codes for modeling the electrochemical response of corroding electrodes. These systems model both the steady state current-potential or polarization behavior of the electrodes and the ac impedance spectra. During this FY, this effort has focussed on modeling corroding systems where two cathodic reactions, oxygen reduction and hydrogen evolution, can occur at the electrode. Since both reactions affect the $\mathrm{pH}$ at the electrode surface, rather complex interactions can take place in the diffusion layer adjacent to the electrode surface. In this layer, the electrolyte can become acidic or alkaline, depending on reaction rates and mass transport conditions. The results of these simulations compare favorably with experiment as shown in figure 4. Some deviations should be expected as the starting assumptions used in the modeling are relatively simple and these deviations are more obvious in the impedance spectra as shown in figure 5 . In the future, this work will address more complicated systems utilizing more realistic assumptions. The ultimate goal is to develop systems for analyzing electrochemical corrosion measurements based on fundamental physical processes and for computer codes that model actual corrosion processes allowing for accurate lifetime prediction.

Corrosion Issues in Nuclear Waste Management

A. C. Fraker, C. G. Interrante ${ }^{*}$ U. Bertocci, E. Escalante, K. J. Coakley ${ }^{\star *}$, E. Plante, E. N. Pugh and R. E. Ricker

* Nuclear Regulatory Commission

** Statistical Engineering Div., Center for Computing and Applied Mathematics

This program was completed during this fiscal year and the database and the associated reports were delivered to the U. S. Nuclear Regulatory Commission (NRC) and to the NRC sponsored Center for Nuclear Waste Regulatory Analyses at the Southwest Research Institute in San Antonio, Texas. The Corrosion Group evaluated corrosion issues relevant to materials used in high level nuclear waste storage. NRC regulations require that nuclear waste shall be substantially completely contained for a period of 300 to 1000 years and that, thereafter, no more than one part in $10^{5}$ of the inventory of radionuclides present at 1000 years after closure may be released annually from the engineered barrier system of a geologic repository. Approximately ninety percent of the waste will be spent fuel (Most $\mathrm{UO}_{2}$ in Zircaloy cladding with three percent in stainless steel cladding.) and approximately cen percent will be embedded in glass. There are potential problems and many concerns relating 
to properties of the glass, the $\mathrm{UO}_{2}$ fuel, the metal canister material and metal cladding. This program included the following activities:

(1) Critical evaluation of scientific research data, measurement techniques, postulated failure mechanisms, and life prediction methodology used in modeling the engineered barrier system of the repository.

(2) The development of a data base system for the convenient examination of the results of the critical evaluations. This data base had 1491 entries and 117 completed reviews when it was transferred to the NRC in June, 1991.

(3) Three experimental projects into basic corrosion processes pertinent to materials and conditions in the repository. The three experimental projects were: (1) effects of transport and resistivity on corrosion, (2) evaluation of methods for detection of stress corrosion crack propagation in fracture mechanics specimens and (3) corrosion behavior of Zircaloy nuclear fuel cladding.

(4) The preparation of interpretative review papers on three different subjects: (1) Mechanisms of Localized Aqueous Corrosion of Copper and Its Alloys, (2) Mechanisms of Stress Corrosion Cracking, and (3) Mechanisms of Internal Corrosion of Spent Fuel Rods

This work provided an opportunity for NIST scientists to pursue the basic objectives of the corrosion effort and the personnel involved in this project served the NRC as a scientific resource helping to prepare the NRC for the difficult decisions that will be required for the safe long term storage of high level nuclear waste.

\section{Advanced Materials}

R. E. Ricker, U. Bertocci, E. Escalante, A. C. Fraker, D. Gram* J. L. Fink, J. V. Nielsen* and M. R. Stoudt

* Guest Scientist, The Engineering Academy of Denmark, Lyngby, Denmark

Influences of Moisture and Pressure on Polymer Matrix Composites - The Corrosion Group evaluated the hypothesis that exposure of polymer matrix composites to high pressure water would result in a permanent degradation in the mechanical properties of the composite. To test this hypothesis, the Corrosion Group exposed a glass fiber reinforced-epoxy matrix (GFRP) composite and a graphite reinforced-epoxy matrix (CFRP) composite to water at ambient pressure and at an elevated pressure. The mechanical properties of the composites were measured before exposure to water, immediately after exposure to water and after removal of the absorbed water. Absorption of water reduced the strength of the GFRP, but had no significant effect on the properties of the CFRP although the appearance of the fracture surfaces was altered by absorbed water as shown in figure 6 . The pressure of the exposure had no significant effect on the composites. For the GFRP, it was shown that the observed reduction in strength was completely reversible upon removal of the absorbed water. As a result, it was concluded that the initial hypothesis is not valid for the systems examined (sponsored by the Office of Naval Research). 
Corrosion of Nickel Aluminide - The corrosion behavior of ductile nickel aluminide has been examined in acidic solutions, over the range of electrode potentials where the transition from active to passive behavior takes place. Impedance spectra have shown that the kinetics in this region is quite complex but not unlike that of nickel in the same conditions. The important conclusion, from a practical viewpoint, is that no significant differences have been found between pure $\mathrm{Ni}$ and $\mathrm{Ni}_{3} \mathrm{Al}$, even during the critical transition when the passive film begins to form. These results, together with a detailed examination of the electrochemical behavior in neutral solutions, confirms the conclusion previously reached that there is no significant difference in corrosion resistance between $\mathrm{Ni}$ and $\mathrm{Ni}_{3} \mathrm{Al}$. This result should encourage commercial users, since it makes the corrosion resistance of nickel aluminide reasonably predictable (sponsored by the Department of Energy, Advanced Industrial Materials Program and The Engineering Academy of Denmark).

Evaluation of Weld Zone Cold Cracking Susceptibility in HSLA Steels - The Corrosion Group, in collaboration with the Navy's David Taylor Research Center, conducted a study of the corrosion and cold cracking resistance of welded high strength low alloy (HSLA) steels. For this study, samples of an HSLA-100 and an HY-100 alloy were heat treated to represent the thermal histories of the parent metal and of the heat affected zone which develops during welding. The corrosion behavior was evaluated by conducting electrochemical polarization experiments and the cracking resistance was evaluated by conducting slow strain rate tensile tests in artificial seawater at constant electrode potentials and under free corrosion conditions (sponsored by the Office of Naval Research).

Metal-Matrix Composites - A series of electrochemical measurements was taken on aluminum specimens in which a SiC single fiber was embedded. These specimens had been annealed for different times to determine if chemical reactions between the metal and the carbide could result in a greater tendency to localized corrosion. The results showed no sign of pitting at the metal-carbide interface. When, by introducing chlorides in solution and raising the electrical potential of the specimen, pitting occurred, it was never at the metal-carbide boundary. It is interesting that, in the course of this study, it was found that the SiC fiber had a not insignificant electrical conductivity, presumably because of doping during its preparation. It is therefore planned to measure the potential that such a fiber would assume by itself in aqueous solution, in order to evaluate the possibility for galvanic corrosion (sponsored by the Office of Naval Research).

Corrosion and Stress Corrosion Cracking of Ductile Iron Aluminide - During this FY, the Corrosion Group initiated a program into the corrosion and stress corrosion cracking behavior of iron aluminide in collaboration with Oak Ridge National Laboratory. During this FY, the Corrosion Group conducted electrochemical experiments on iron aluminide in neutral and acidic solutions. During the coming FY, the Corrosion Group will compliment this work with slow strain rate tests in these solutions. Other solutions will be added to the study so that the behavior of this material over the normal range of pH's and potentials will be evaluated (sponsored by the Department of Energy, Advanced Industrial Materials Program). 
Corrosion Behavior of Magnesium Alloys - Magnesium alloys are light weight materials that promise to provide significant improvements in the efficiency of engineering systems that use moving parts. However, more information is needed on the fundamental processes that determine the corrosion behavior of these materials. Currently, the salt spray test is used almost exclusively for evaluating the corrosion behavior of these alloys. This test is useful for emulating severe service conditions but it provides very little information on the electrochemical processes that cause failure. As a result, the Corrosion Group is conducting conventional electrochemical experiments on four commercial $\mathrm{Mg}$ alloys (2 produced by rapid solidification followed by powder metallurgy and extrusion (RS/PM) and 2 die cast), some binary metallic glasses and one metal-matrix graphite fiber composite in different solutions to determine the effects of alloy composition and processing (rapidly solidified, die cast, etc.) on the fundamental processes that determine the corrosion behavior. The ultimate objective is the development of more corrosion resistant $\mathrm{Mg}$ alloys and composites and better corrosion testing methods for $\mathrm{Mg}$ alloys and composites. 


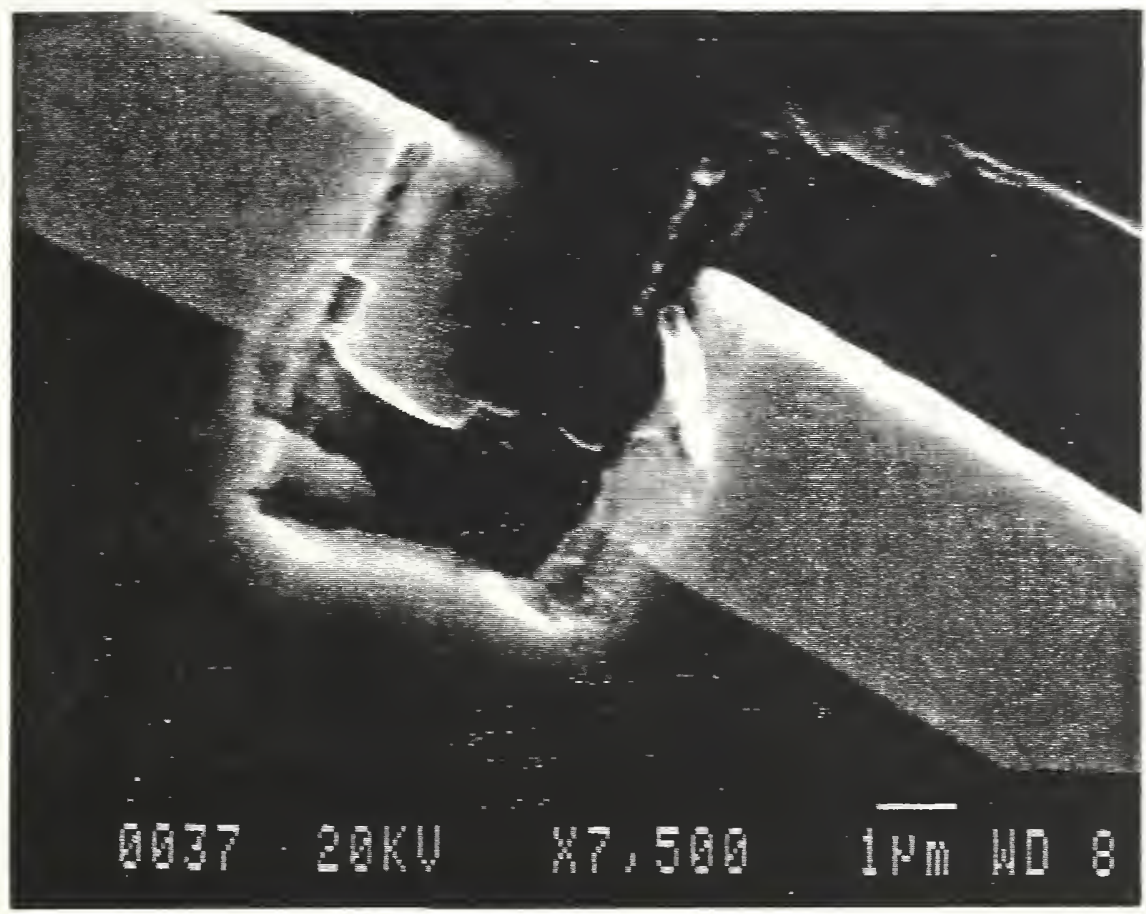

Figure 1. Rhodium plated nickel after bending. Crack appears to have penetrated into nickel 1 to $1.5 \mu \mathrm{m}$ before arresting.

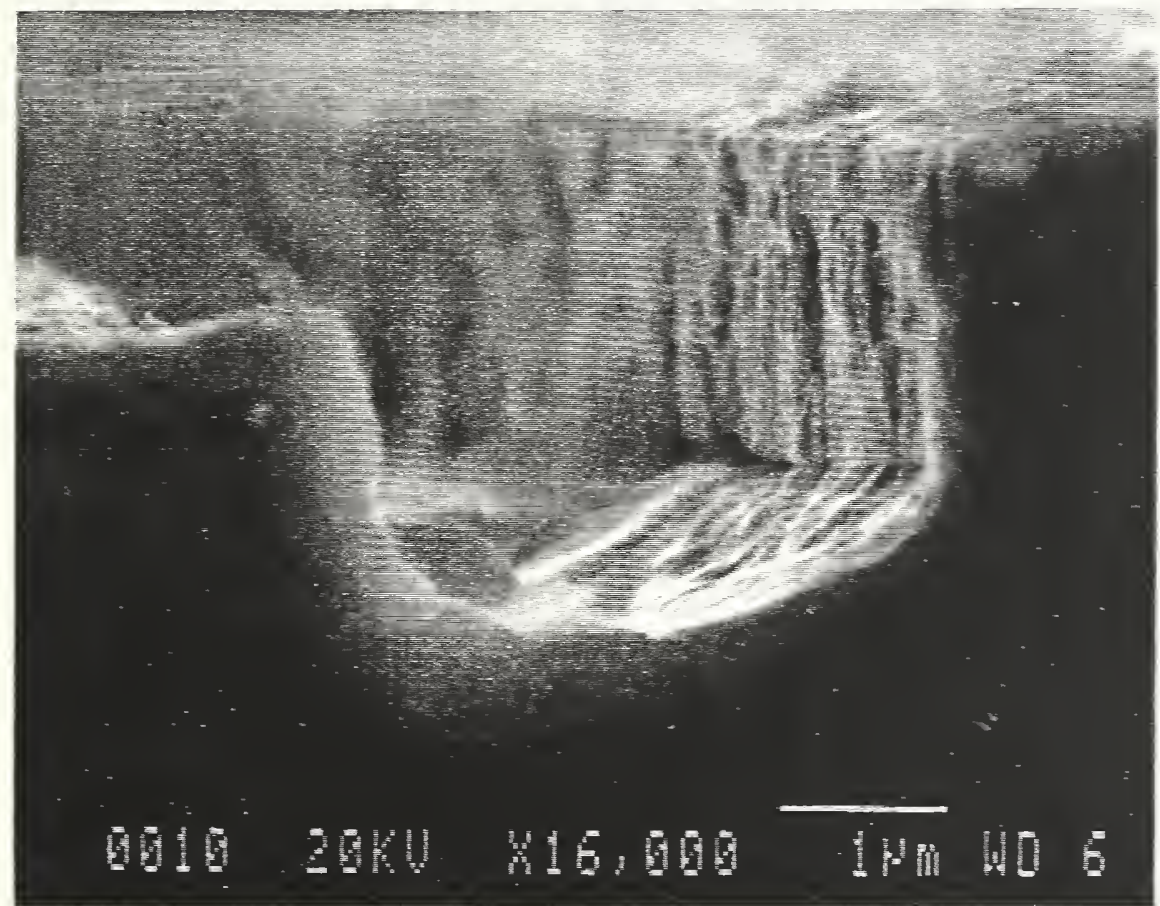

Figure 2. Rhodium plated nickel after bending. Crack appears to have arrested at nickel/rhodium interface. 


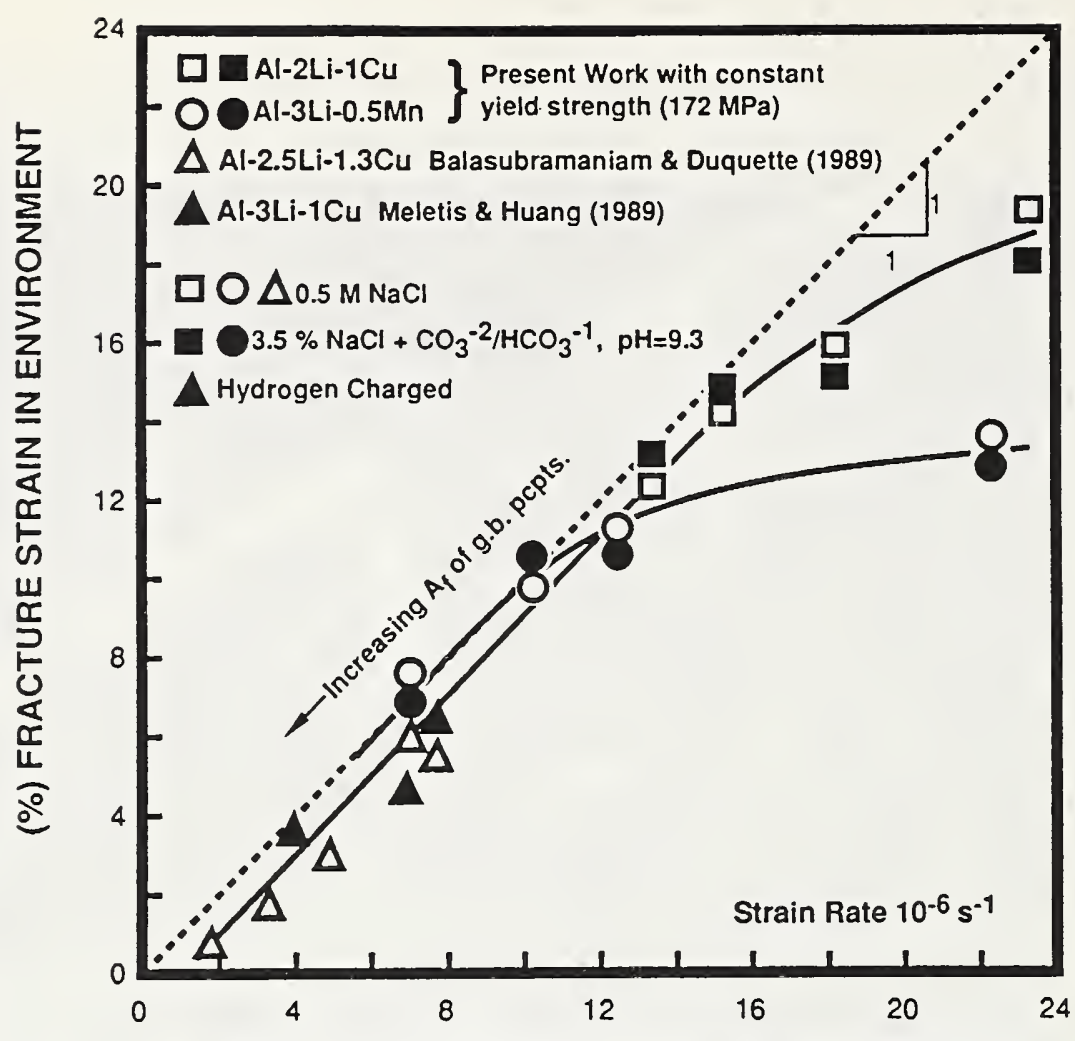

(\%) FRACTURE STRAIN IN AIR OR DRY NITROGEN

Figure 3. Slow strain rate test results for an $\mathrm{Al}-\mathrm{Li}$ and an $\mathrm{Al}-\mathrm{Li}-\mathrm{Cu}$ alloy with different heat treatments but with the same yield strength and slip character.

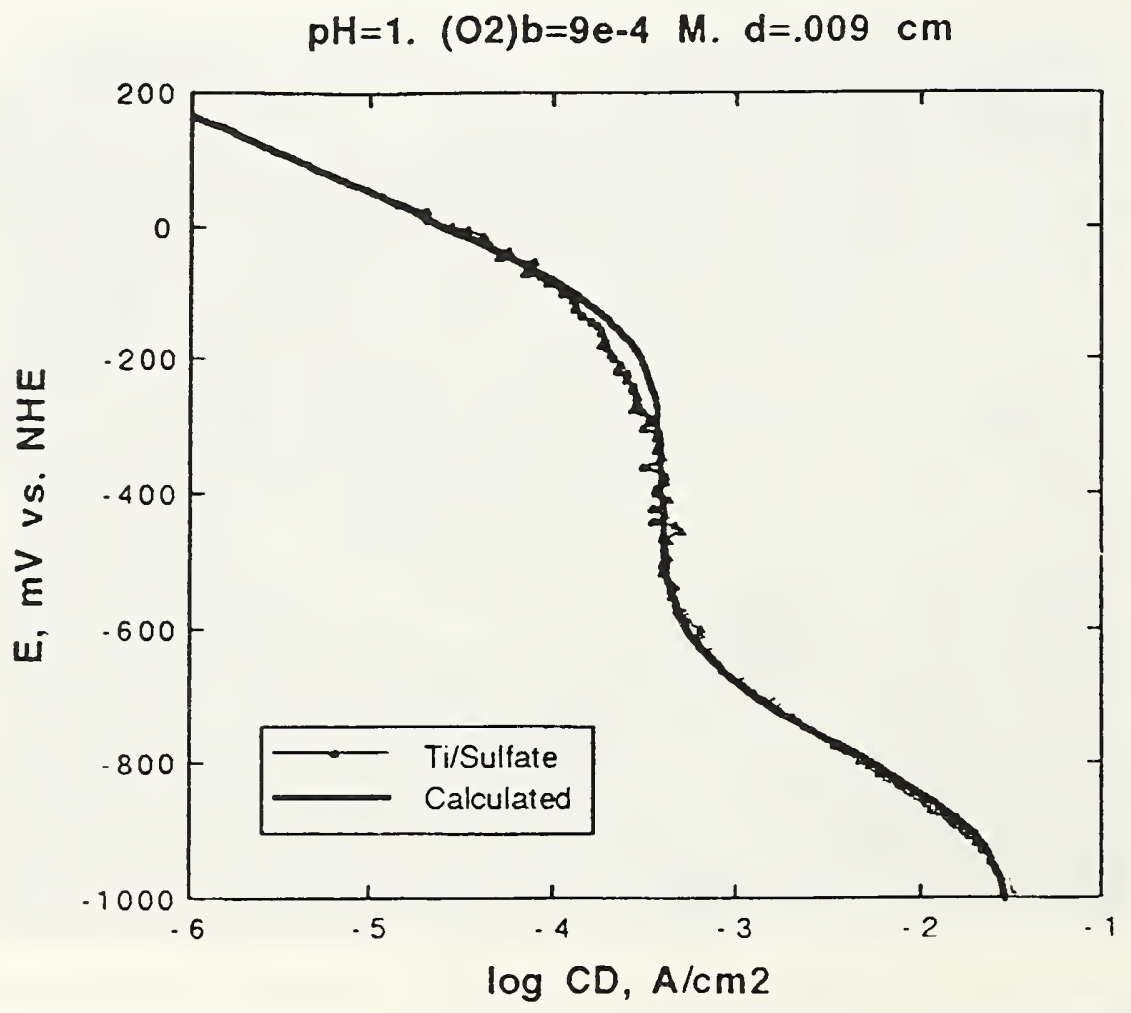

Figure 4. Comparison of the cathodic polarization behavior of $\mathrm{Ti}$ in a sulfate solution to the calculated behavior. 

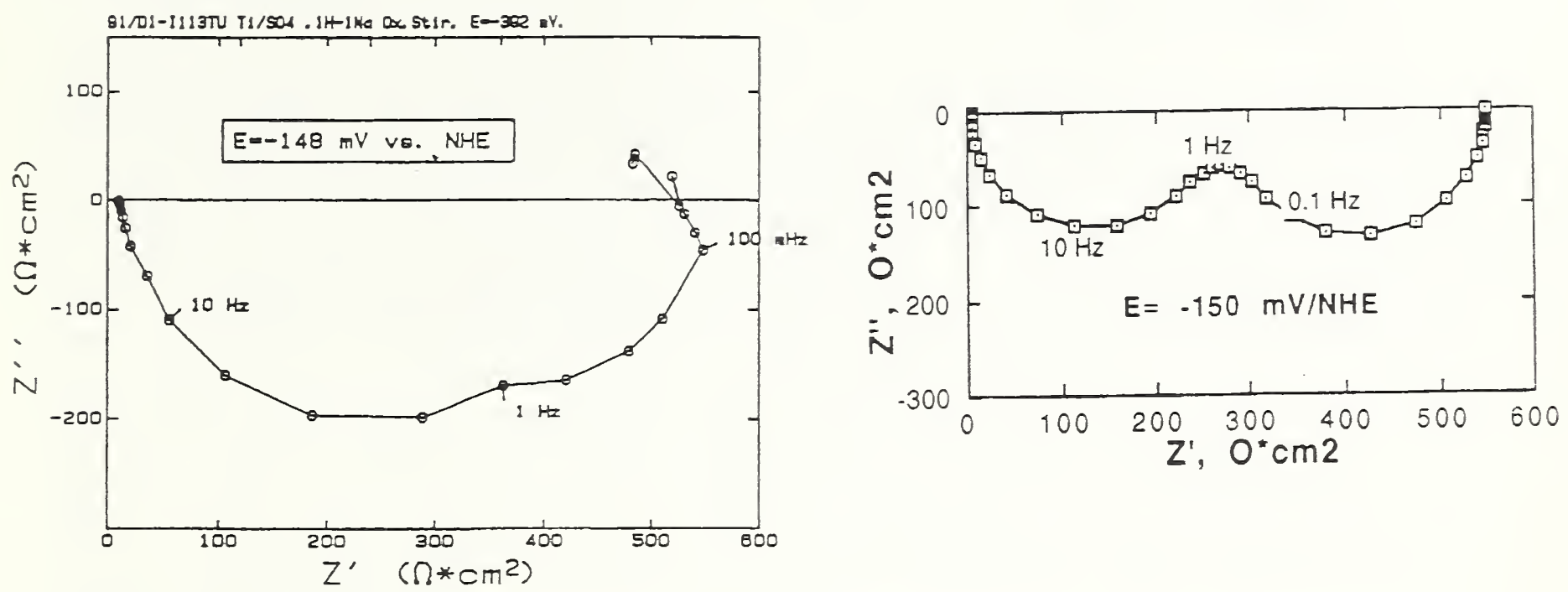

Figure 5. Comparison of the impedance of a $\mathrm{Ti}$ electrode in a sulfate solution to the calculated behavior.

\section{CFRP in WET CONDITION $90^{\circ}$ Fiber Orientation}
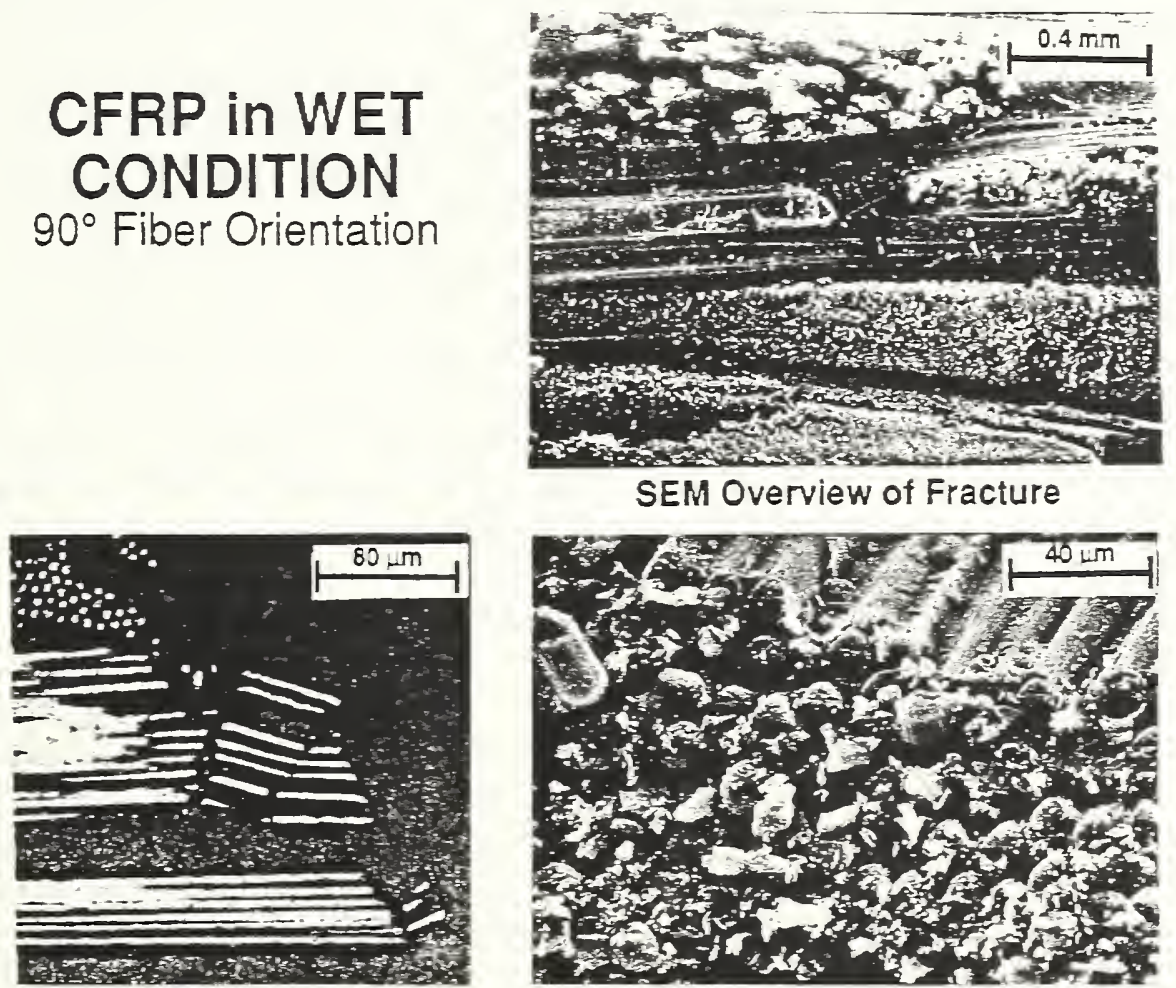

Cross section

Compression Failure . "Fiber Chopping"

Figure 6. Scanning and optical micrographs showing the influence of absorbed water on the fracture behavior of a graphite-epoxy composite. 
1

1

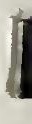

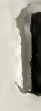

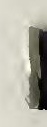

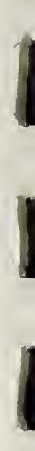

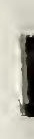

I

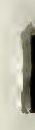

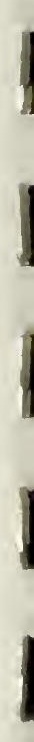


The Electrodeposition Group is responsible for measurements and standards associated with electrodeposited alloys. The objectives of the group are: (1) the determination of the critical mechanistic, materials, and process variables controlling the structure/property relationships of electrodeposited coatings and the development of approaches that will result in improved materials and processing controls for industry; (2) the provision of standards such as coating thickness standards, dye penetrant crack standards, tin-lead alloy standards, stage micrometer for SEM calibration and corrosion step test standards; (3) the development of new standards requiring electrodeposition for their fabrication or utilizing the unique properties of electrodeposited alloys; (4) the provision of NIST expertise to voluntary standards organizations, (5) the provision of NIST expertise to industry, through research associates, appropriate contracts and consulting arrangements, and (6) the provision of NIST expertise to other government agencies through appropriate contracts.

The following are some of the many areas in which alloy coatings are important for the commerce of the United States: (1) Strategic Materials - It has been shown that appropriate alloy coatings can provide a 30 percent savings of imported raw chromium. Research on new alloys indicate that for many applications, coatings can replace bulk stainless steels. (2) Corrosion Electrodeposited coatings play an important role in corrosion protection. It has been estimated that the cost of corrosion to the U.S. economy is in excess of 200 billion dollars per year. (3) Wear - The cost of wear to the U.S. economy has been estimated at about 50 billion dollars per year. Electrodeposited coatings play an important role in improving wear properties and surface coatings can be optimized for particular wear situations. Electrodeposition Industry - Electrodeposition, specifically plays an essential role in the United States economy. For example, almost 900K tons of electrogalvanized sheet and strip are produced annually with an estimated impact of about $\$ 150 \mathrm{M}$ per year. Approximately $550 \mathrm{~K}$ tons of metal coated wire and wire products are produced per year. Tin plate accounts for 8800 tons with a dollar impact of $\$ 120 \mathrm{M}$, and foil production accounts for about $\$ 200 \mathrm{M}$ per year (5) Magnetic Materials - Most hard disk drives are produced utilizing electrodeposition technology thus indicating the important impact of electrodeposition on the United States computer industry. (6) Electronic Materials - All printed circuit boards use electrochemical deposition in many stages of their fabrication including lead frames, electric contacts and through hole plating. (7) Processes - Manufacturing processes including decorative coatings, electroforming (compact discs), and electronic packaging (contacts, PC boards, etc.) are so important that without electrodeposited coatings much of our current industry would not be able to function in its present form. (8) Advanced Composites - The Wilson Report on Materials Policy, Vol. 3, No. 29 April 1991 estimated a market of 20 billion dollars per year by 2000 for advanced composites. The market for aircraft engines, as given by the U.S. Industrial Outlook 1986, was $\$ 17$ billion dollars and growing by $\$ 1$ billion per year. This area of technology is important for the U.S. balance of trade - about $\$ 3$ billion of U.S. exports are accounted for by this market and together with $\$ 96$ billion in aerospace exports. High speed 
deposition of alloys with near atomic control of interface composition and deposition of intermetallics can play an important role in this area of advanced technology.

\section{FY91 Significant Accomplishments}

- A new trivalent chromium plating electrolyte has been developed which shows promise of alleviating the pollution problems associated with the hexavalent electrolyte.

- Significant progress has been made in developing a continuous fiber plating line to electroform matrices of titanium aluminide onto moving fibers or tows.

- Compositionally modulated alloys (CMA) of cobalt copper and chromium cobalt have been produced electrochemically.

- A new understanding of how misfit dislocations nucleate in artificial superlattices has been obtained.

- New information on the growth of titanium aluminide intermetallics may shed general light on nodular formation in general in electrodeposited alloys.

- Solder standards are now in large scale production.

- High quality Co-Cu multilayers are now being electrochemically produced.

\section{Electrodeposition and Electron Microscopy of Intermetallic Coatings}

N. S. Wheeler, C. T. Sarafidis*, and D. S. Lashmore

* Guest Scientist - Harry Diamond Laboratories

The purpose of this project is: (1) to observe and to characterize the nucleation and growth of copper-tin intermetallics which form between copper and $t$ in in situ, and (2) to electrodeposit both $\mathrm{Cu}_{6} \mathrm{Sn}_{5}$ and $\mathrm{Cu}_{3} \mathrm{Sn}$ to act as possible diffusion barriers. An understanding of the nucleation and growth processes would benefit the electronics industry, since the morphology of these intermetallic layers and their passive films are thought to have a key role in the failure of solder joints.

Soldering in industry is plagued by the oxidation of copper-plated component leads and the copper portions of the printed circuit (PC) boards. This is a problem, since lead/tin solder will not adhere to an oxidized surface. In order to avoid oxide formation, those parts requiring soldering are pretinned, meaning that a tin coating is plated or melted onto the copper surfaces to prevent copper oxidation. However, during storage at room temperature, $\mathrm{Cu}_{6} \mathrm{Sn}_{5}$ tends to form at the tin-copper interface as a result of interdiffusion. If the pretinning is accomplished at higher temperatures by dipping in molten metal, then both $\mathrm{Cu}_{6} \mathrm{Sn}_{5}$ and $\mathrm{Cu}_{3} \mathrm{Sn}$ layers are formed. Later, when the tin coating is reflowed with solder to form a joint, these intermetallic layers may cause dewetting. Then, once the solder joints are formed and the PC 
board is in use, the joints may fail for reasons that are not well understood. The hypothesis explored in this investigation suggests that the manner in which these intermetallics are formed, their passive films, and their morphology may all have an effect on the reflow process and the lifetime of the solder joint.

Transmission electron microscopy (TEM) is ideal for observation of nucleation, since it is capable of the necessary atomic resolution. The approach proposed for the nucleation studies is to heat a tin-coated copper substrate in the TEM, allowing the intermetallic formation to be observed in situ by looking through the tin, intermetallic, and copper layers. A method has been devised for producing the appropriate specimens for this experiment, and the TEM study will be carried out during FY'92.

The approach taken for the $\mathrm{Cu}-\mathrm{Sn}$ intermetallic deposition is twofold. The first study is to determine the effect of the intermetallic morphology on the structural integrity of the interface during exposure to heating and cooling, and the parts of this study include: (1) development of electrolyte/s from which one or both of the intermetallic phases of interest can be directly electrodeposited, (2) use of this electrolyte to deposit a smooth intermetallic coating on a copper substrate, (3) electrodeposition of tin on top of the intermetallic coating, (4) annealing of the sample, and (5) microscopic observation of the interface. This study has resulted in the development of a non-cyanide $\mathrm{Cu}-\mathrm{Sn}$ electrolyte from which deposits containing approximately 35 to 45 atomic-percent tin have been electrodeposited. These compositions were deposited on a copper-coated mild steel panel using a Hullcell, an asymmetric electrochemical cell in which the anode and the sample panel are not parallel but positioned at an angle to one another. This arrangement sets up a current density gradient across the panel. Thus, if the tin composition varies with current density, it will also vary with position on the panel. The Cu-Sn deposition was followed by deposition of tin and, finally, annealing at $200^{\circ} \mathrm{C}$ for $24 \mathrm{hr}$ in a vacuum. The micrographs, shown in Figure 1 , of cross sections of three portions of this panel, whose as deposited tin concentrations were approximately 45, 42, and $37 \%$, show that one portion of the panel was much less friable during mechanical polishing, suggesting that the $37 \%$ copper-tin deposit might be more ductile. Further research is in progress to determine the factors that preserved the structural integrity.

The second study, to determine solderability, will include: (1) electrodeposition of a smooth coating of $\mathrm{Cu}_{6} \mathrm{Sn}_{5}$ intermetallic (or, alternatively, the $\mathrm{Cu}-\mathrm{Sn}$ composition which was found to be the most effective in the first study), (2) electrodeposition of solder on top of the Cu-Sncoated copper, (3) reflowing the solder, and (4) testing the solderability and mechanical strength of this layered structure by comparison with solder applied to a pretinned copper substrate having no intermediate Cu-Sn electrodeposit. 
A new deposition cell designed for the deposition of titanium aluminum alloys on moving fibers has been developed. This fourth generation cell incorporates a built-in turbo pump developed at NIST which forces a stream of electrolyte at right angles to the moving fibers. This pump provides an increase in deposition rate approximately a factor of 12 over the previous generation cell. In addition this new cell allows the fibers to be run in a straight line rather than forcing them to bend into the liquid. This approach dramatically facilitates rethreading a cell in case of fiber breakage. In fact this cell can easily be rethreaded even at high temperatures. The heat uniformity is insured by placing the entire apparatus in an oven the temperature of which can be controlled to within about $2{ }^{\circ} \mathrm{C}$. To suppress the ingress of oxygen and water vapor the cell incorporates two argon airlocks which have gold plated fiber brushes incorporated, eliminating the need for liquid mercury contacts. The cell is constructed from a glass teflon composite and contained in a pressurized pyrex glass cylinder. It can operate at temperatures up to $300{ }^{\circ} \mathrm{C}$. Experimental alloys of titanium aluminum manganese have been produced on $\mathrm{W}$ wire to simulate silicon carbide fibers. These wires have been consolidated into composites by hipping and an example of such a composite is shown in Figure 2.

\section{Electrochemical Deposition of Titanium Aluminum Intermetallics}

G. R. Stafford

Several approaches have been used to introduce $\mathrm{Ti}^{+2}$ into a chloroaluminate electrolyte for the purpose of electrodepositing Al-Ti alloys in the 25-50 at.\% $\mathrm{Ti}$ range. These include the in-situ synthesis of $\mathrm{Ti}\left[\mathrm{AlCl}_{4}\right]_{2}$ and the direct electrochemical dissolution of titanium. The highest $\mathrm{Ti}^{+2}$ concentration achieved thus far is $240 \mathrm{mM}$; this is a $70 \%$ increase over that which had been obtained last year. Unfortunately, electrodeposition from this electrolyte does not result in an appreciable increase in the titanium content of the alloys, which contain 28-30 at.\% $\mathrm{Ti}$ and are fcc with the ordered $\mathrm{Ll}_{2}$ structure. Depositing from this more concentrated electrolyte does, however, allow one to deposit $\mathrm{Al}_{3} \mathrm{Ti}$ at acceptable current densities by eliminating diffusion limitations of $\mathrm{Ti}^{+2}$.

The electrodeposition of $\mathrm{Al}_{3} \mathrm{Ti}$ from a chloroaluminate electrolyte containing $\mathrm{Ti}^{+2}$ proceeds at current efficiencies greater than $95 \%$. This has been determined both gravimetrically and by stripping voltammetry. In addition, by studying the current transient at the beginning of potentiostatic deposition, we have determined that $\mathrm{Al}_{3} \mathrm{Ti}$ forms by progressive 3-dimensional nucleation followed by hemispherical diffusion controlled growth. Unlike the deposition of pure aluminum from these electrolytes which is limited by instantaneous nucleation( only occurs during the initial transient and is followed by growth of those nuclei) the addition of titanium promotes nucleation. The nucleation rate increases dramatically with overpotential; correspondingly, a decrease in grain size is seen with increasing overpotential.

Electrodeposits of $\mathrm{Al}_{3} \mathrm{Ti}$ from chloroaluminate electrolytes containing $\mathrm{Ti}^{+2}$ show extensive \{111\} twinning and growth striations parallel to \{111\} are observed 
in sub-stoichiometric (low $\mathrm{Ti}$ ) deposits. The presence of these striations suggests that the alloy does not grow continuously, but rather by forming many thin layers of identical crystallographic orientation which grow on each other. The presence of coherent twin boundaries throughout the electrodeposits, and particularly at the corners of nodules, suggests that the twins play a significant role in deposit growth. This hypothesis is consistent with the solidification behavior of materials with high entropies of melting, where liquid/solid interfaces are atomically flat and require ledge motion to grow. Potential sources of ledges include surface nucleation sites (above the critical size), screw dislocations, and twin intersections; the latter two sites are permanent sources since they are not consumed by the ledge growth. While it is not possible to ascertain the cause of the \{111\} twins in these electrodeposited titanium aluminides, the morphology of the nodules suggests that the twins act as a source of ledges, with crystal growth emanating from the twin plane.

A study of phase formation in electrodeposited Al-Mn alloys from chloroaluminate electrolytes containing $\mathrm{Mn}^{+2}$ has shown that alloys containing 48-55 at.\% $\mathrm{Mn}$ and deposited at temperatures below $300^{\circ} \mathrm{C}$ are the $\tau$ phase (stoichiometrically $\mathrm{Al}_{11} \mathrm{Mn}_{14}$ ). The crystal structure of $T$ is $\mathrm{Ll}_{0}$ (CuAu structure), an imperfectly ordered face-centered cubic (fcc) variant in which the (002) planes are alternately Al and Mn. The superlattice reflections are clearly visible in $x$-ray diffraction patterns. Increasing the deposition temperature above $300^{\circ} \mathrm{C}$ results in the appearance of the equilibrium $\delta_{2}$ phase, and at temperatures exceeding $350^{\circ} \mathrm{C}$, the deposit is single phase $\delta_{2}$ (by $\mathrm{x}$-ray diffraction). Three equilibrium phases in the Al-Mn system ( $A{ }_{6} \mathrm{Mn}^{\mathrm{M}}$, $\mathrm{Al}_{11} \mathrm{Mn}_{4}$ and $\delta_{2} \quad \mathrm{Al}_{8} \mathrm{Mn}_{5}$ ) have now been electrodeposited directly, but temperatures in excess of $250-350{ }^{\circ} \mathrm{C}$ are required.

\section{Particulate Composites}

D. R. Kelley and C. E. Johnson

A particulate composite coating consisting of tetra boron carbide particles $\left(B_{4} C\right)$ incorporated in a nickel matrix has been produced. As shown in Figure 1 , these composites contain 40 to $45 \%$ of $1 \mu \mathrm{m} B_{4} C$ particles. Table 1 shows the results of accelerated wear tests, and demonstrates that these composites have a significantly lower wear rate compared with engineering or hard chromium. The deposition process has been scaled up to coat this particulate composite alloy on large parts.

\section{Deposition of Chromium from a Trivalent Electrolyte}

C. E. Johnson, E. Soltani, and D. S. Lashmore

The use of chromium in the hexavalent state results in rather expensive waste treatment procedures to reduce if not eliminate highly toxic and carcinogenic waste products. This unfortunate situation can be avoided by adopting currently available trivalent chromium deposition technology. Chromium is rarely deposited commercially from trivalent electrolytes because most commercial processes are only capable of producing limited thickness. These commercial coatings are not nearly as resistant to wear and have a different surface appearance than the widely used hexavalent chromium. One obvious problem with deposition of chromium from the trivalent state are anodic 
reactions which usually give rise to the oxidation of $\mathrm{Cr}^{+3}$ to $\mathrm{Cr}^{+6}$. This oxidation if allowed to occur gives rise to reduced current efficiency, and increases in waste treatment costs. The oxidation reaction can be selected to be bromine or chlorine evolution, which occurs at a lower anodic potential than does $\mathrm{Cr}^{+3}$ oxidation with the obvious penalty of dealing with toxic bromine gas. We are investigating an electrolyte containing formate ions which at $\mathrm{pH} 1.5$ yields $0.0736 \mathrm{~kg} / \mathrm{amp}-\mathrm{s}$. compared with a measured 0.010 to $0.0127 \mathrm{~kg} / \mathrm{amp}-\mathrm{s}$ for the common hexavalent electrolyte. This trivalent electrolyte is about 6.8 times more energy efficient than the hexavalent.

Samples of trivalent chromium deposited from a formate based electrolyte were $\mathrm{x}$-rayed on an $\mathrm{x}$-ray diffractometer and the results shown in Figure $4 \mathrm{a}, \mathrm{b}$, and c illustrating 3 clear amorphous peaks. This spectra is now being analyzed to help us understand more about the amorphous nature of these alloys. Upon heat treatment at $200{ }^{\circ} \mathrm{C}$ the pattern first suggests bcc chromium, then most probably a carbide or oxide mixed with chromium. At $600{ }^{\circ} \mathrm{C}$ the existence of the carbides or oxides are more apparent.

It is unusual to produce a single element metallic glass. We therefore strongly suspect that the formate ion $\left(\mathrm{CO}_{2} \mathrm{H}^{+}\right)$is being incorporated with the chromium in the deposit. We shortly will be conducting some wavelength dispersive $x$-ray studies to determine this. The current efficiency (C.E.) of the electrolyte seems to range from a maximum of $33 \%$ to about $15 \%$ and is strongly dependent on the current density (and on overall mass transport). As the transport increases for example on a rotating disk electrode from 100 to $1000 \mathrm{rpm}$, the C.E. drops about 20\%. This behavior suggests that a film of some type forms on the cathode and blocks the reaction. From the observation that we also are producing an amorphous alloy, and probably also incorporating something into the deposit, it is tempting to conclude that this film consists of an oxidized formate species.

\section{SRM Coating Thickness Standards}

H. B. Gates, D. R. Kelley and D. S. Lashmore

The Office of Standard Reference Materials (OSRM) now has in stock at least a years supply of most of our thickness standards currently in production. Two hundred lead-tin standards have been produced. The final statistical analysis of results is pending, with initial estimates of error being 8 to $10 \%$ for both mass/area and composition. We are awaiting shipment of packaging material

before delivery to OSRM. Development of the automation of the copper on steel is continuing with efforts this past year on automating the wavelength dispersive $x$-ray florescence instrument. A new laboratory has been designed and its installation is complete. This installation incorporates significantly improved ventilation, production electroplating machines of our own design which were fabricated for NIST by a Baltimore firm, and automated sample turning to improve current distribution. Plans are underway to improve our hexavalent chromium plating facility which has become outmoded. 
Artificial Superlattices

D. S. Lashmore, C. R. Beauchamp, J. Mullen, and E. Soltani

Modulated alloys of cobalt copper, copper nickel and cobalt chromium have been produced this past year and their properties are being studied. The focus of the IBM program has been on the magnetic properties of artificial

superlattices of all three systems and the Wright Patterson program on the mechanical properties. Success in understanding plastic deformation in thin modulated materials has been made. For example we have shown that misfit dislocations can be formed as a result of an interaction between dislocations emitted from some appropriate source, say a crack, and the interfaces. The mechanism proposes that a single source will result in only every other layer becoming incoherent. Such behavior has been observed and is illustrated in the TEM micrograph of Figure 5 of a copper nickel CMA with a $10.0 \mu \mathrm{m}$ periodicity. If the layer thickness is larger, say about $300 \mu \mathrm{m}$, then every layer pair should be incoherent as is shown in Figure 6 .

New techniques to test the mechanical properties of thin foils are being developed in collaboration with Dr. Richard Fields. The technique consists of electroforming a cylinder of the multilayer on a copper mandrel, cutting the material on the mandrel to remove the high current density edges, then finally stripping the copper away from the CMA to leave a free standing cylinder. This cylinder is now amenable to testing for both the elastic and plastic properties. Elastically the cylinder can be tested in compression to yield a Young's modulus and then in tension to study the elastic/plastic behavior of the multilayer. The advantage of this type of tensile geometry is that the cylinder will deform elastically to very large strains, thereby allowing precise measurements to be made on relatively simple equipment. In tension this geometry eliminates grip effects.

The electrodeposition of cobalt-copper multilayers of approximately $3 \mathrm{~nm}$ wavelength for each metal has been accomplished on <l00> oriented copper single crystal substrates. A potentiostatic electrodeposition techniques to deposit cobalt copper multilayers has been developed. The technique consists of pulses between $-1.40 \mathrm{~V}$ vs. SCE for cobalt and $-0.65 \mathrm{~V}$ vs. SCE for copper with an $\approx 3$ seconds cell interrupt between the cobalt to copper transition. The purpose of the interruption is to avoid cobalt dissolution. A typical pulse representing the cell interrupt technique following the deposition of a cobalt layer is illustrated in Figure 7. Residence time for the cobalt and the copper is presently determined by means of the coulometric technique developed previously in the copper-nickel program. The composition of the electrolyte employed has been determined as a 100:1 ratio of cobalt to copper and consists of a solution of $0.34 \mathrm{M}$ cobalt sulfate, $0.003 \mathrm{M}$ copper sulfate, and $30 \mathrm{gm} / 1$ boric acid. Other operating conditions have been optimized at a $\mathrm{pH}$ of approximately 3.0, no forced convection while deposition is carried out, and a temperature of $45^{\circ} \mathrm{C}$.

A histogram of the charge passed during the cobalt layer in the sample is represented in Figure 8 demonstrating enhanced control over the deposition process using this technique. It can be seen in this figure that $92 \%$ of the 
layers fall within $3 \%$ of the mean charge. In Figure 9 the diffraction pattern for a sample is presented demonstrating first order satellites on both sides of the multilayer peak and second order satellite on the high side.

This particular sample has demonstrated four fold symmetry around the $z$ axis with respect to ferromagnetic resonance properties, as measured by $\mathrm{Dr}$.

McMichael and confirmed by Dr. Bennett. This is the first time that such a phenomenon have been observed on multilayered samples.

Multilayer alloys of cobalt chromium are now being produced from a single electrolyte. The chromium seems to be deposited as a metallic glass and the cobalt as fcc cobalt. Layers as thin as $2.0 \mu \mathrm{m}$ and as thick as $400 \mu \mathrm{m}$ can be made using potentiostatic deposition under coulometric control. The significance is that cobalt chromium alloys exhibit an anomalous magnetic resistance effect and the capability to electroform these materials provides industry with a low cost process to take advantage of the magnetic behavior of this alloy. Low angle diffraction of the Co-Cr CMA's is shown in Figure 10 illustrating one satellite on this particular sample. 
Table I.

Comparison of the wear rates of various particulate composites with hard chromium and 52100 steel

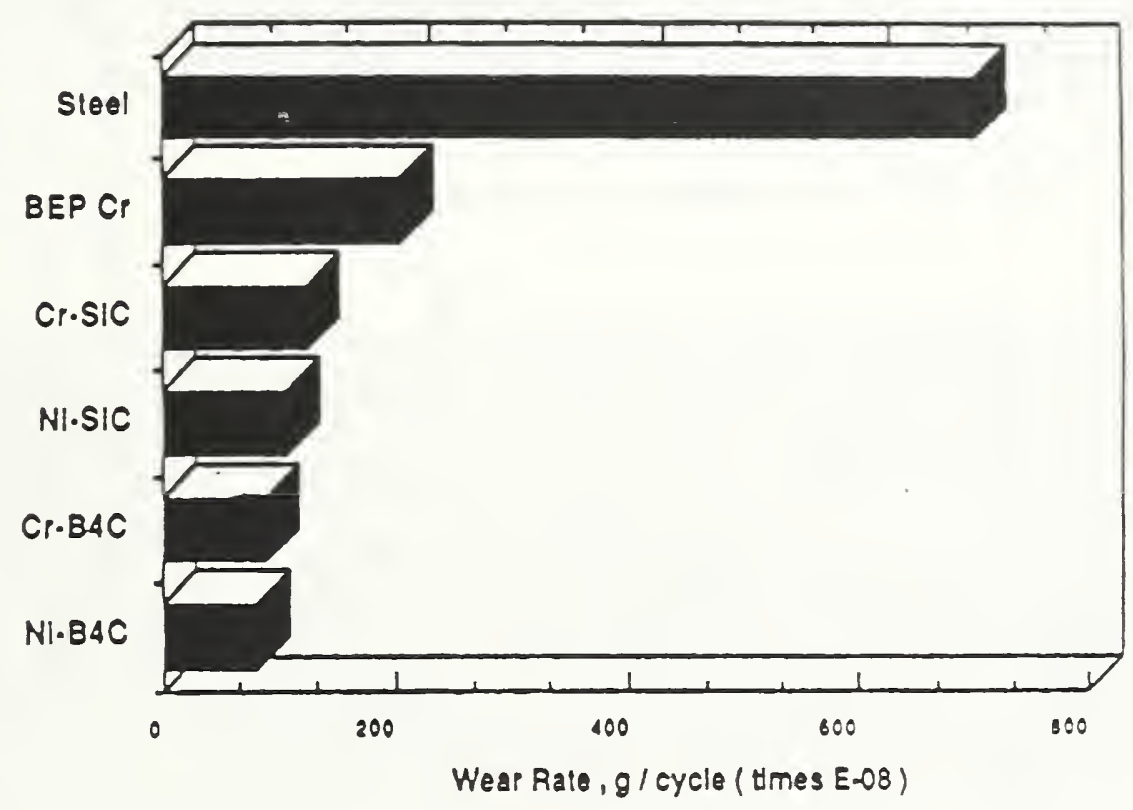



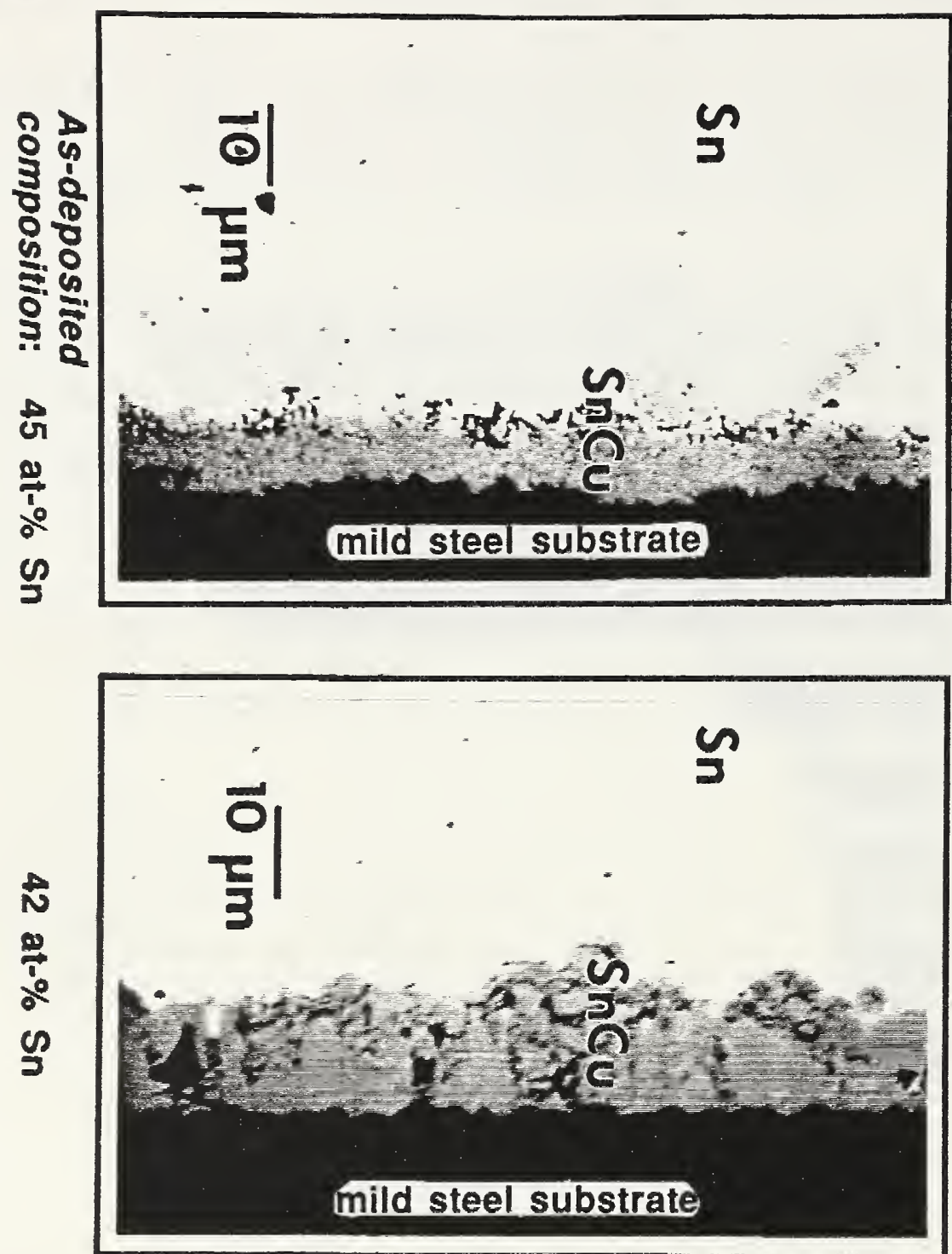

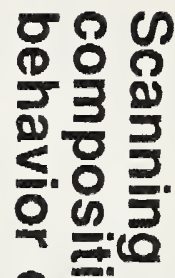

은 $\frac{1}{1}$

10 9

$2=0$ (

훙요

(1)

둥 흘

ต.

Б웅

Q $\frac{20}{0}=$

○舟

$\div 0 \div$

c $\frac{0}{\circ}$ 饮

3 a 5

$\infty \equiv$

क \& 0 政

$\because \pm \frac{1}{10}$

(1)

321

$\boldsymbol{D}=5$

¿ 50

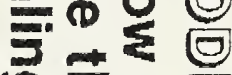

0 \%

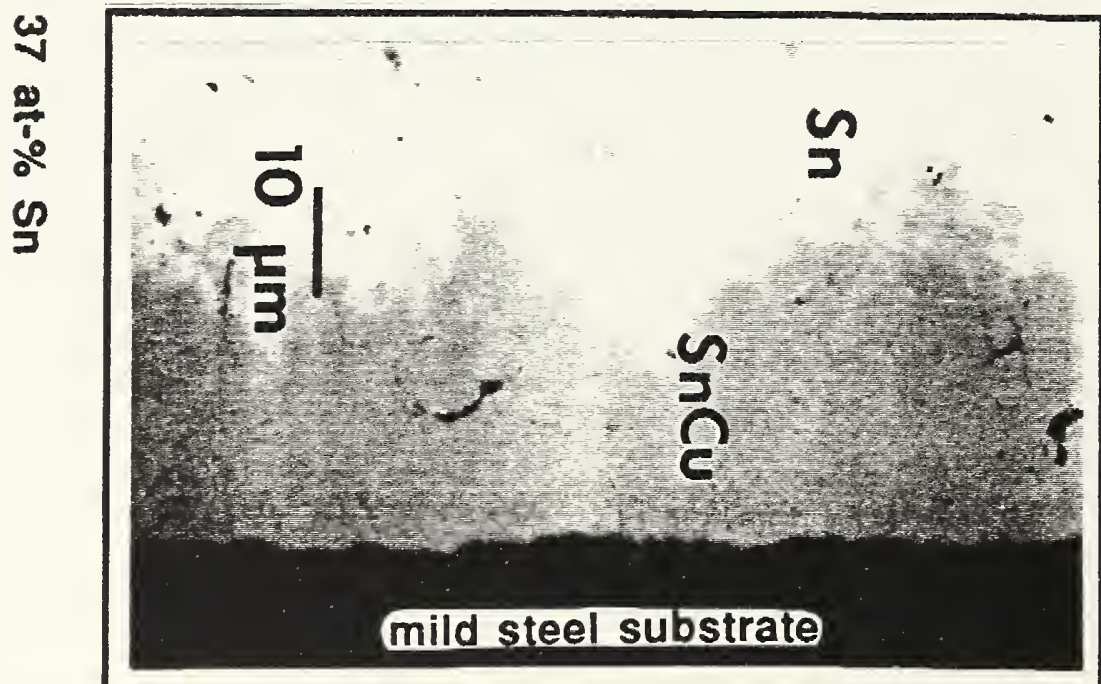

욕

$\therefore 300$

$29=\frac{Q}{5}$

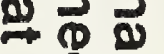

ง

$8 \equiv \frac{0}{8}$

0 .

กำ 


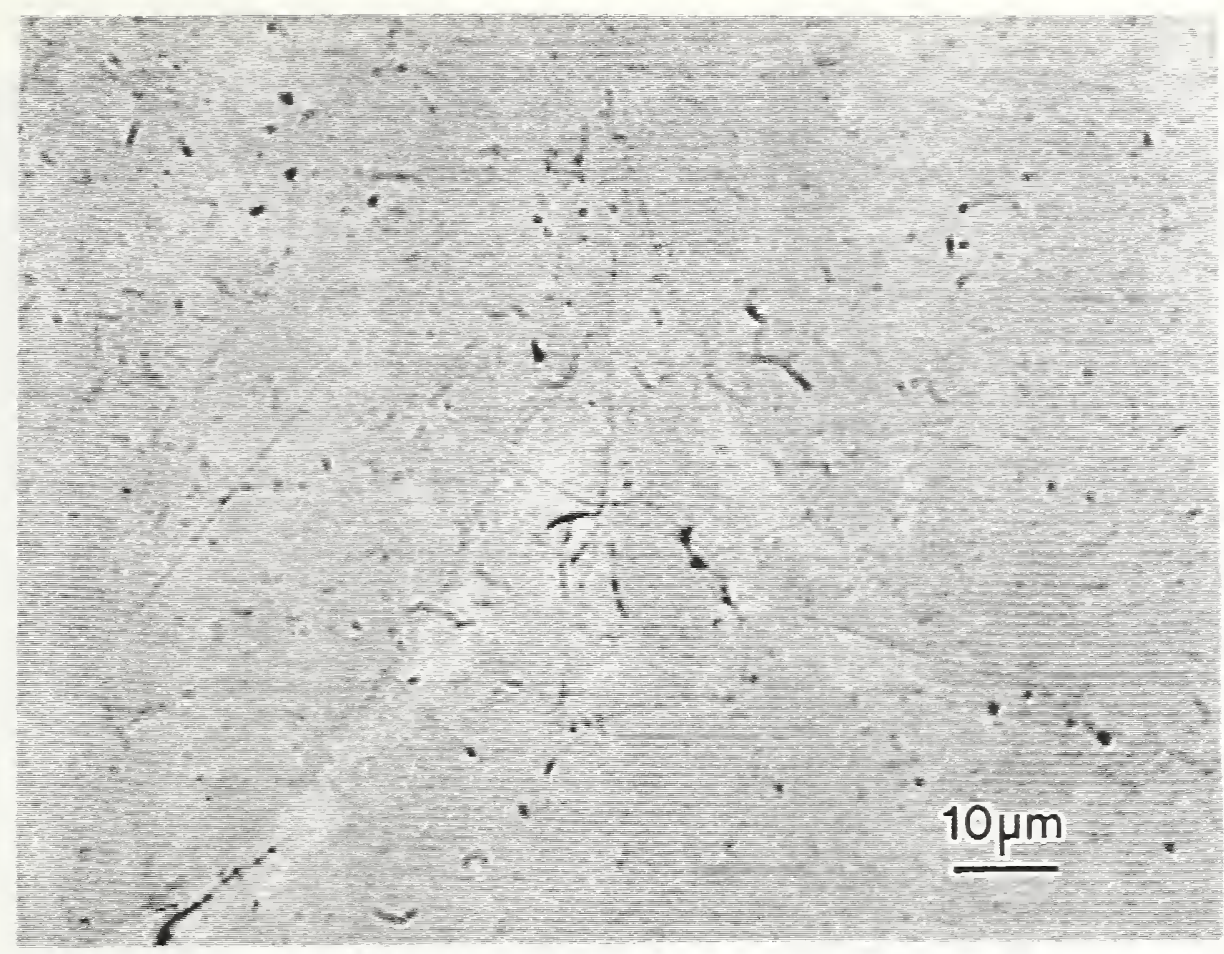

Figure 2. An optical micrograph of a hipped Al-Mn composite reinforced with tungsten wire. $1000 \mathrm{X}$ showing a crack resulting from transformation to $\mathrm{Al}_{6} \mathrm{Mn}$ before complete consolidation.

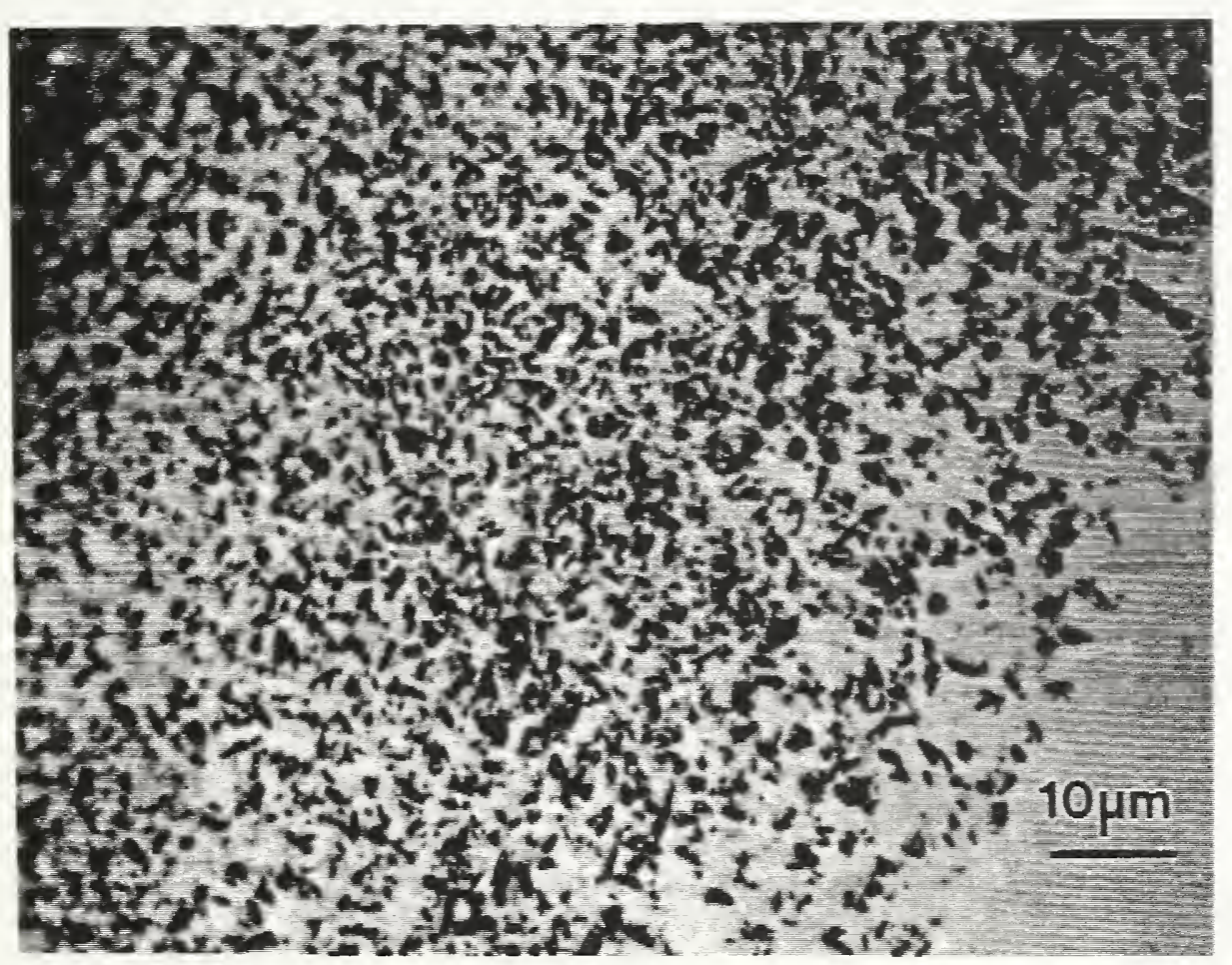

Figure 3. A SEM cross section of a $B_{4} C$ particulate composite. 


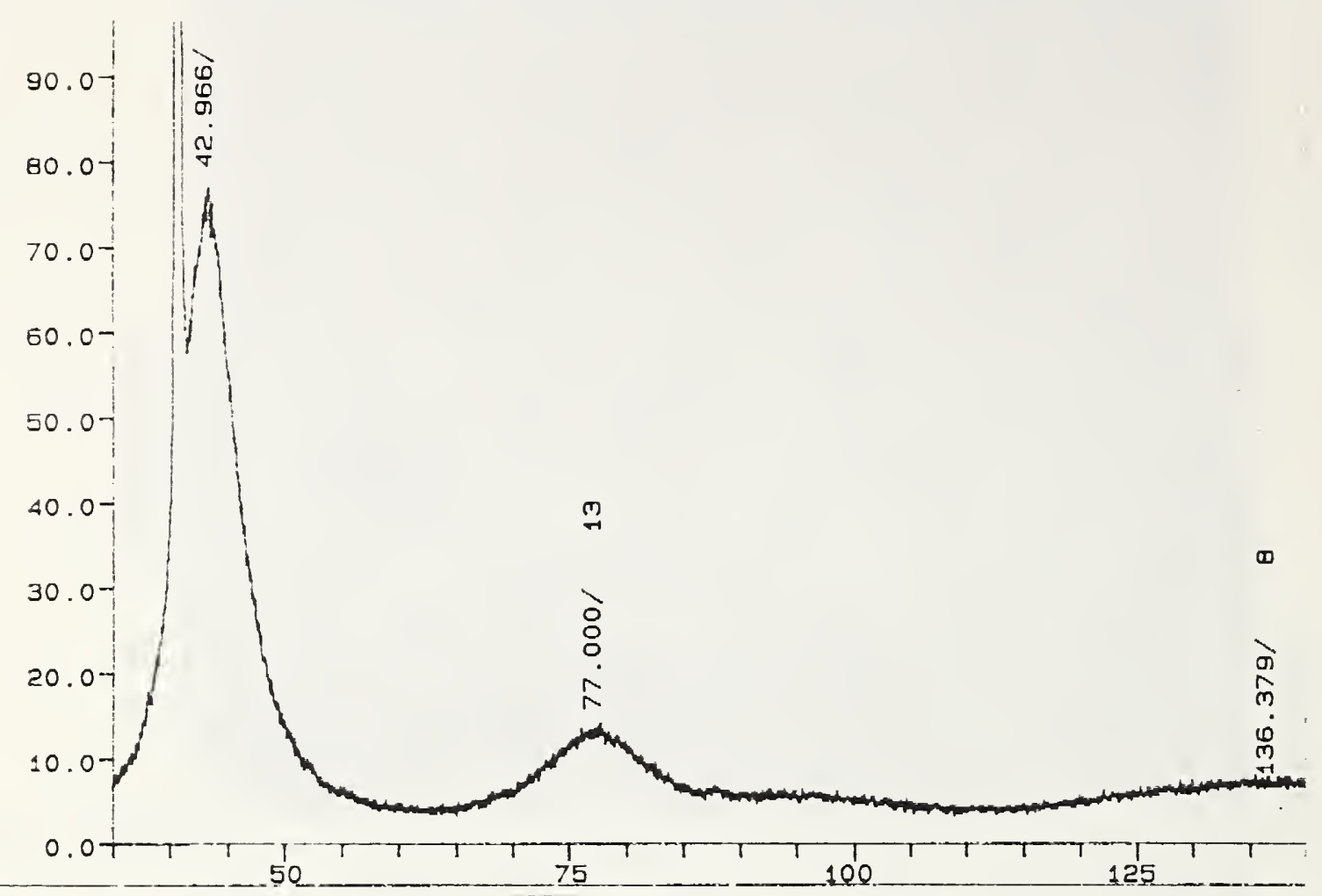

Figure 4. An x-ray diffraction pattern of electrodeposited amorphous chromium showing 3 distinct amorphous peaks.

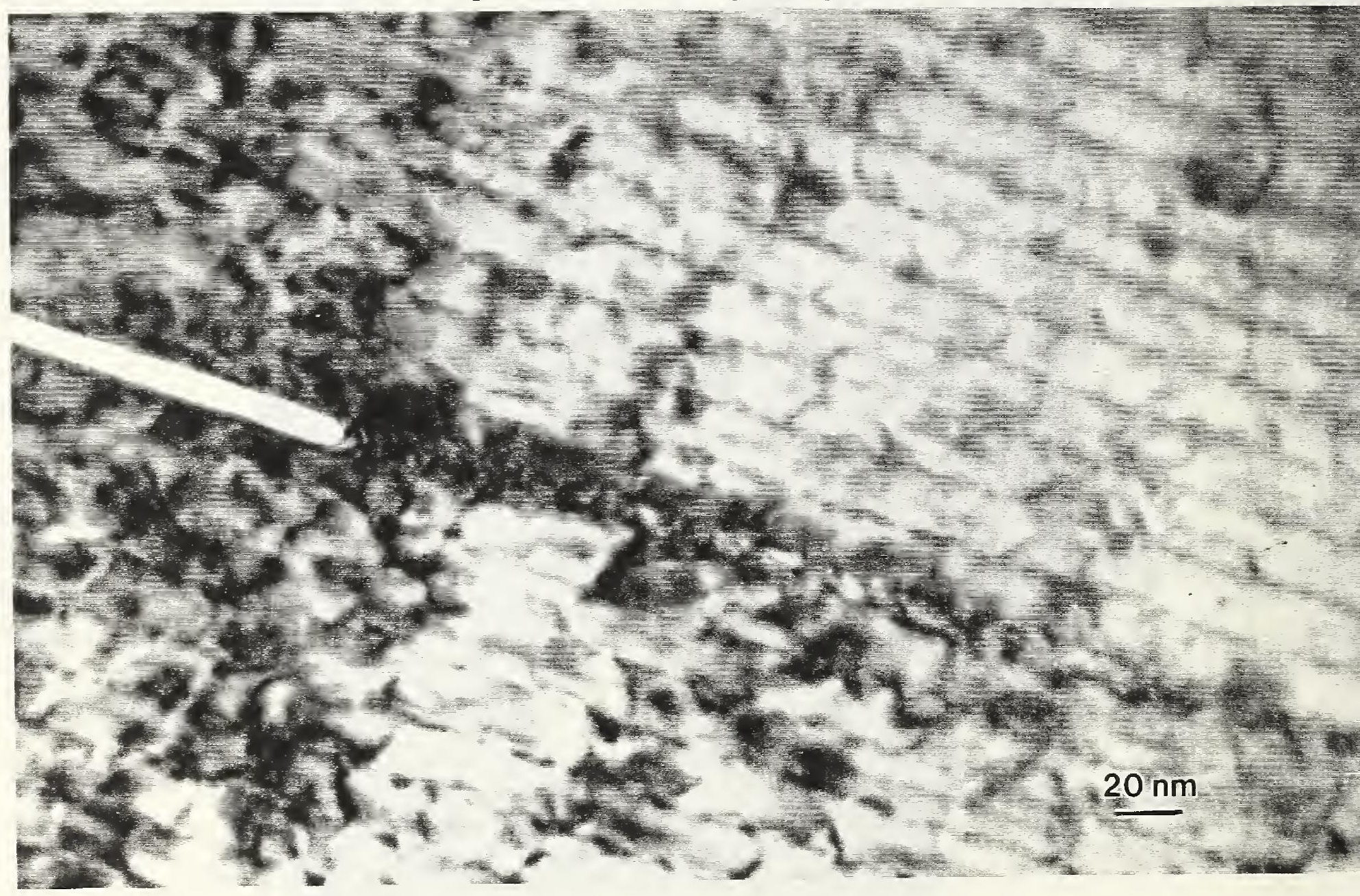

Figure 5. A TEM micrograph at $300 \mathrm{KeV}$ of a CuNi CMA illustrating a crack and associated dislocation structure. The layer spacing is $100 \AA$ for each material. 


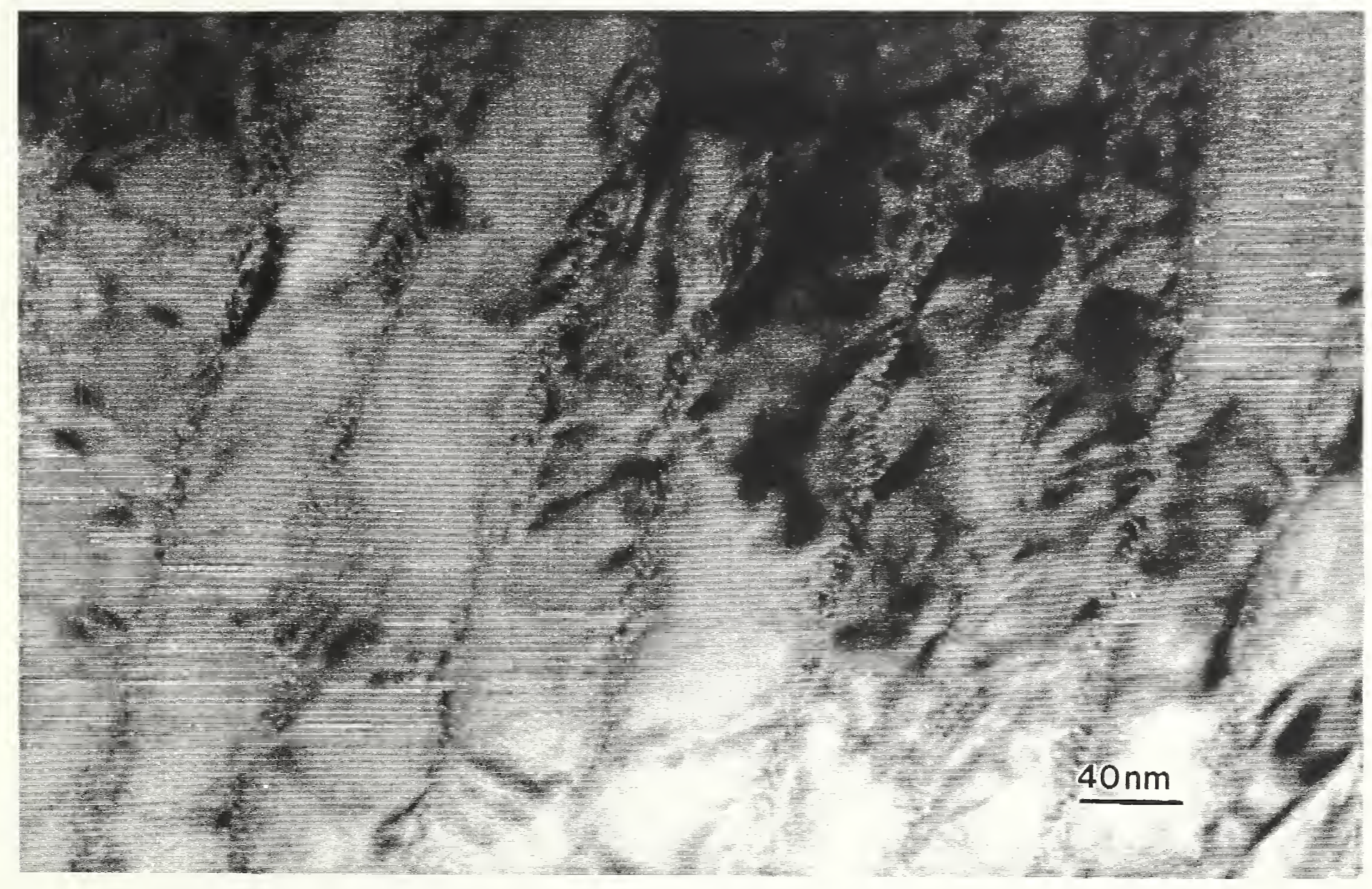

Figure 6. A TEM micrograph of a incoherent CuNi CMA. Each layer is about $300 \AA$. The misfit locations are revealed by the Moire' patterns.

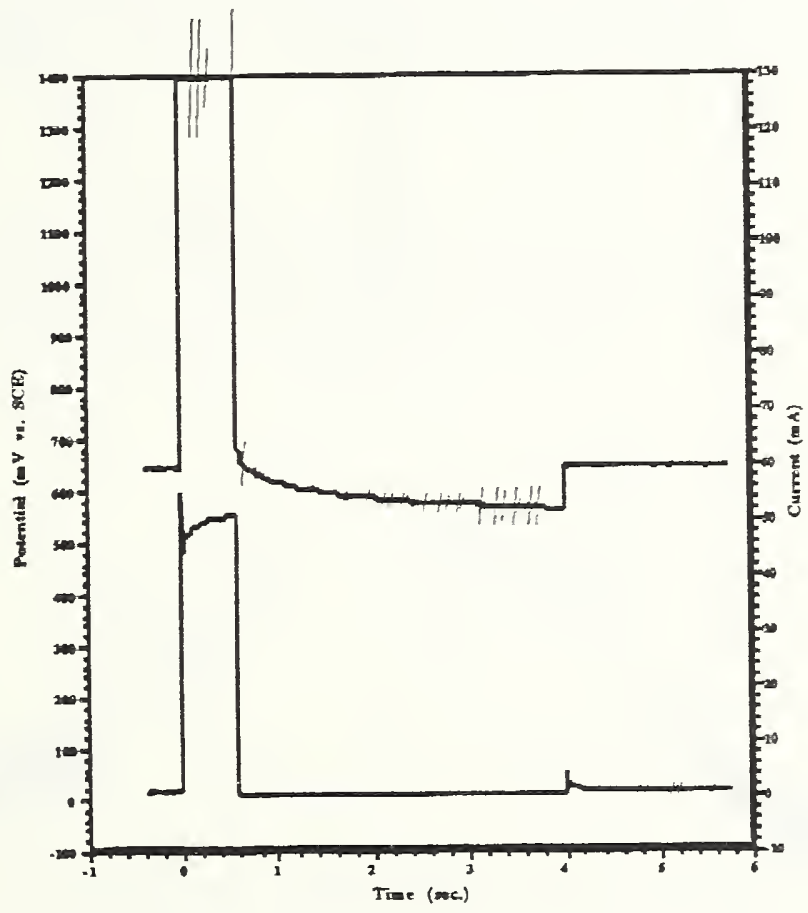

Figure 7. A comparison between applied potentiostatic wave form (upper curve) and the current response (lower curve) for a Co-Cu CMA electrolyte. The upper curve shows a region where the potential is turned off to allow a Cu immersion deposit to form on the cobalt. 


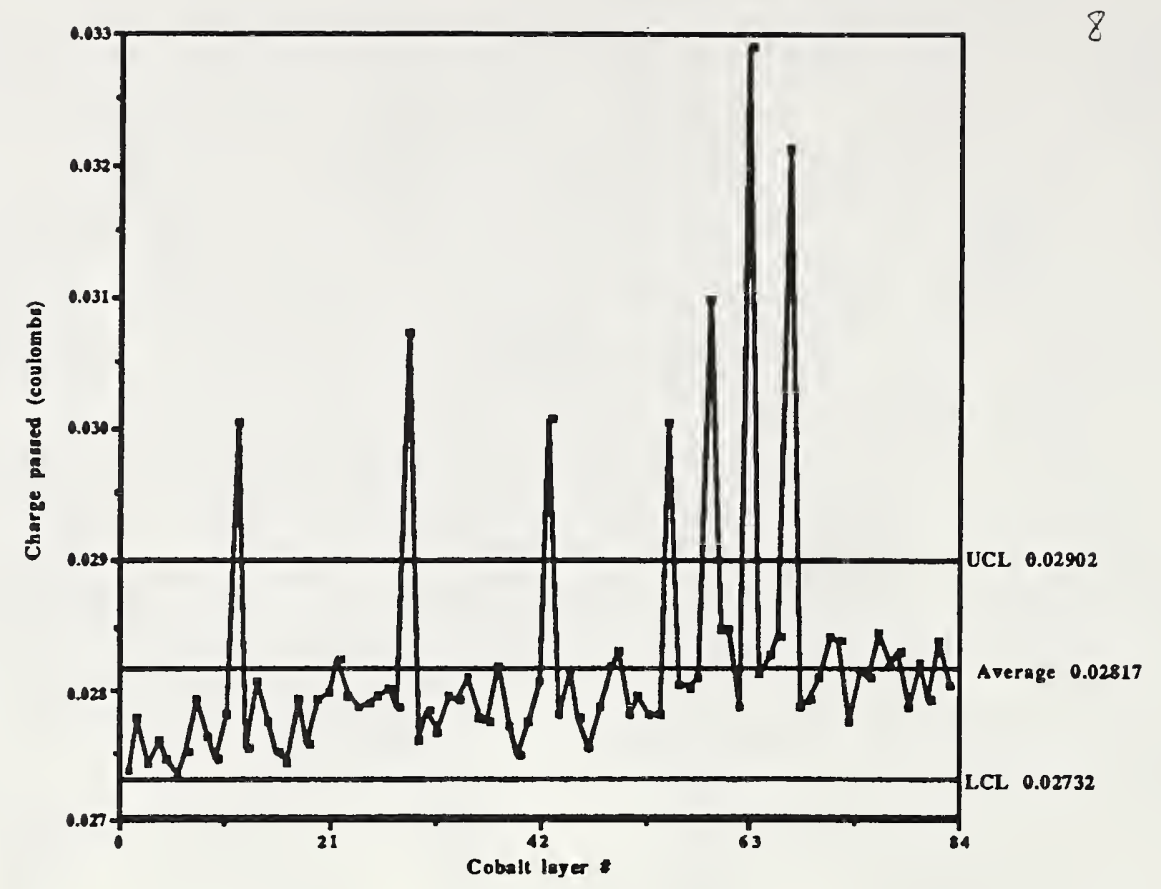

Figure 8. A histogram of the charged passed per layer with a potentiostat wave form under coulometric control for a [200] single crystal substrate.

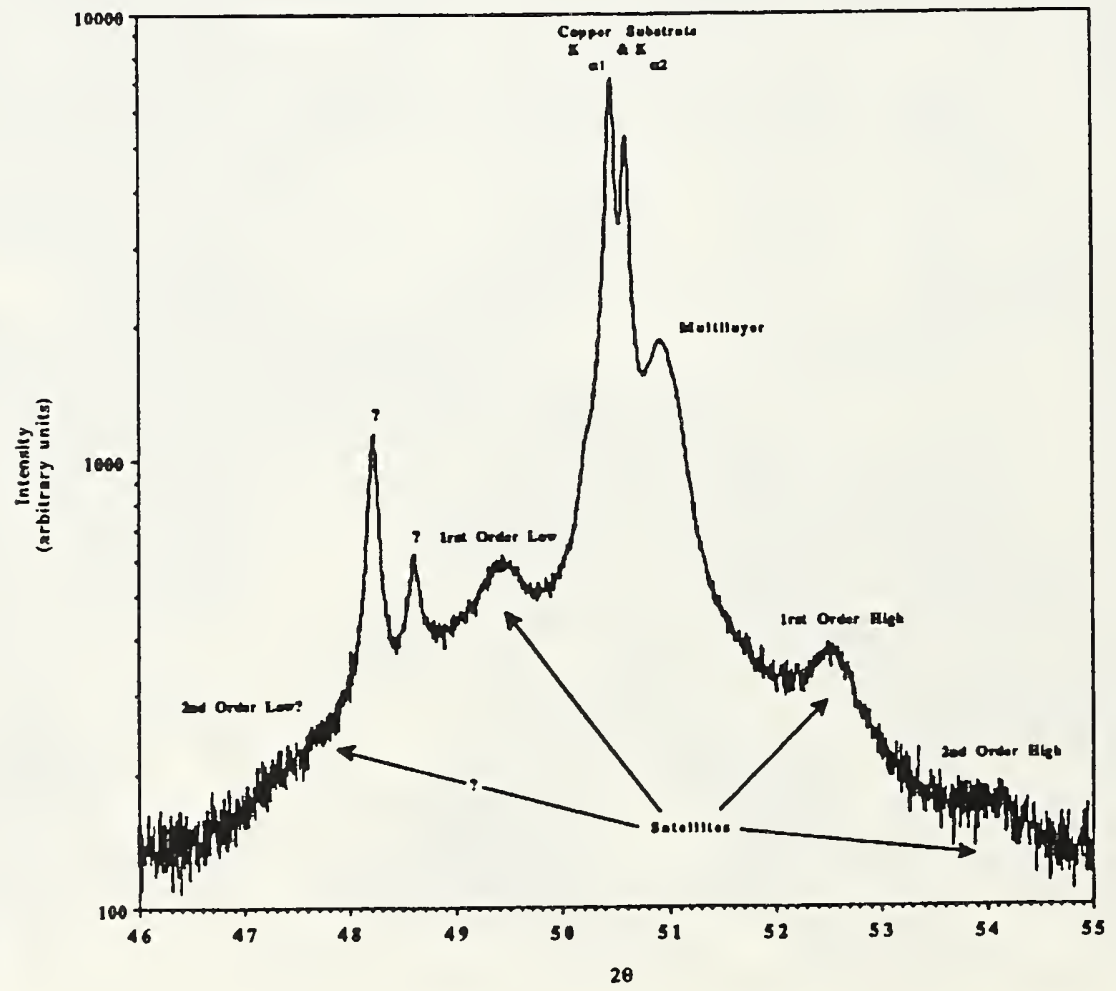

Figure 9. A high angle diffraction pattern of Co-Cu CMA deposited on a [200] copper single crystal showing 2 orders of satellites. 


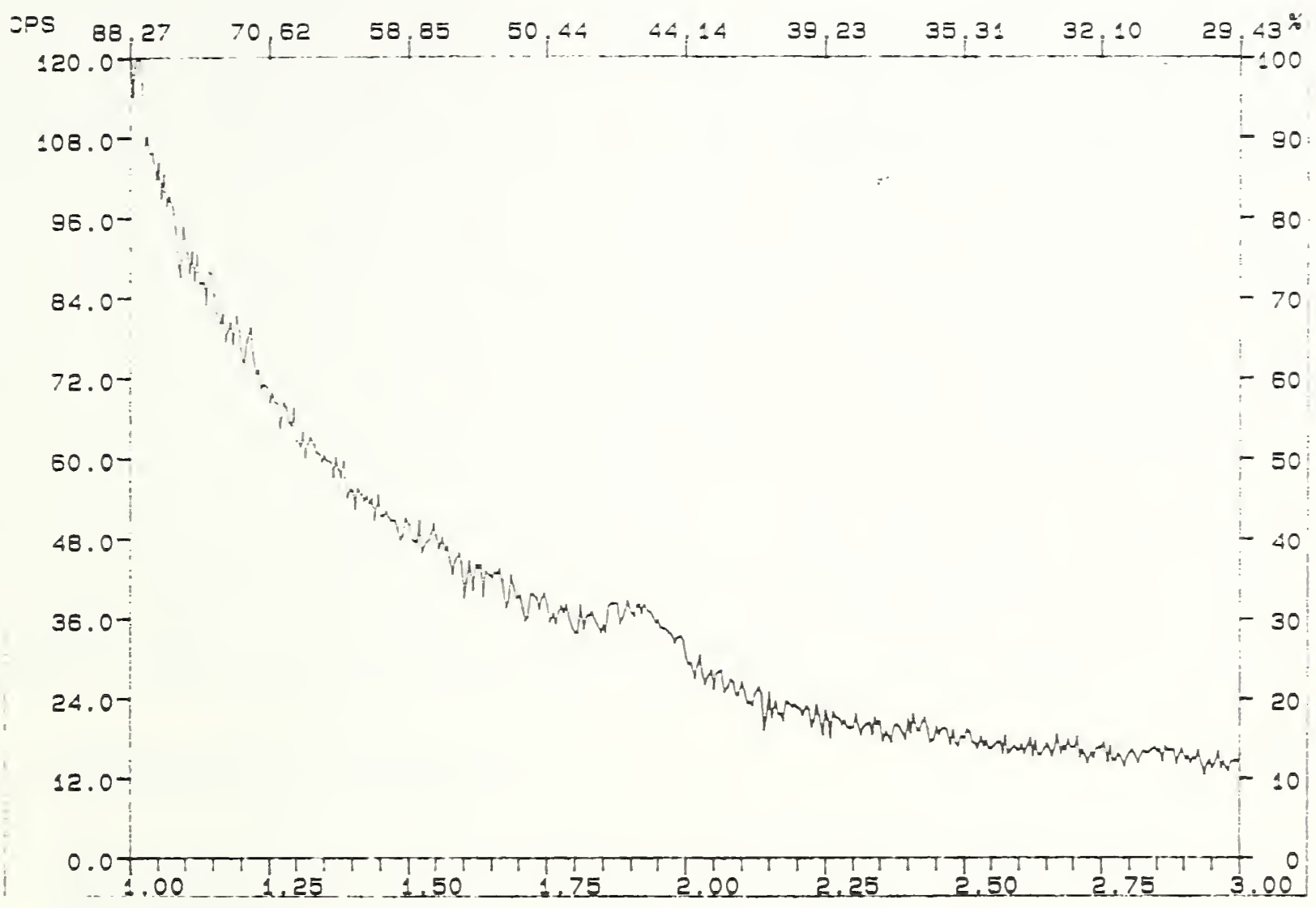

Figure 10. A low angle diffraction pattern of a Co-Cr CMA with one layer amorphous $(\mathrm{Cr})$ and one layer crystalline (Co-fcc). Only one satellite is shown in this pattern. 

The high temperature materials chemistry research program emphasizes the thermodynamic, chemical-kinetic, interfacial microstructure, and molecularlevel behavior of inorganic materials in high temperature process and service environments. The main current objectives are: (1) to develop and apply (e.g., to refractory ceramics and composites) a new molecular-specific methodology for obtaining thermal and chemical stability data at ultra-high temperatures $\left(2000-5000^{\circ} \mathrm{C}\right)$; (2) to develop a unique facility for the controlled production of thin films by laser deposition, coupled with in situ molecular-level measurement devices, for the determination of basic data and process mechanisms, particularly for superconducting, ferroelectronic, and other advanced electronic films; (3) as an extension of objective (2), to laser deposit magnetic thin films and correlate the film properties with the molecular level characteristics of the deposition vapor/plasma phase; (4) to support the U.S. steel industry development of a new direct reduction iron/steel making process, through production of a thermodynamic database and solution model for prediction of slag, refractory, and inorganic inclusion thermochemistry; (5) to carry out mechanistic studies of oxidation for enhancement of the utility of superalloys in high temperature oxidation environments; (6) to develop and apply new or improved measurement methods for the thermodynamic characterization of high temperature vapors at elevated pressures; and (7) to provide technical support to the NIST/American Ceramics Society (ACerS) Ceramic Phase Diagram Data Program through development of computer graphics for computer storage and manipulation of phase diagrams.

\section{FY91 Significant Accomplishments}

- The laser-induced vaporization mass spectrometric (IVMS) technique (developed in-house) was applied to thermodynamic and kinetic studies of vapor plumes produced from refractory carbides ( $\mathrm{SiC}, \mathrm{TaC}$ ), and oxides $\left(\mathrm{HfO}_{2}, \mathrm{Fe}_{3} \mathrm{O}_{4}\right)$. Time resolved $(<\mu \mathrm{sec})$ target temperatures, obtained indirectly from beam velocity analysis and directly by a novel optical multichannel spectral analysis of surface emission, were found to be basically in agreement. Ultrafast signal-averaging ( $>100 \mathrm{Mhz}$ ) and optical multichannel spectroscopy was utilized for the study of nonequilibrium (in addition to equilibrium) processes occurring on the laser-impact time scale of nanoseconds.

- The laser thin film deposition facility was upgraded by the installation of an excimer laser system. In a cooperative project with the Ceramics Division and the US Army Harry Diamond Laboratories (HDL), lead zirconate titanate (PZT) films were deposited on silicon using UV radiation from the excimer laser system. These films exhibited better morphology and ferroelectric properties than films prepared previously using visible or IR radiation from a Nd/YAG laser.

- A new project to characterize the pulsed laser deposition of magnetic thin films from $\mathrm{Ag}-\mathrm{Fe}_{3} \mathrm{O}_{4}$ targets has been initiated in collaboration with the Magnetic Materials Group. Plume species have been identified, using mass and optical spectroscopy, as a function of position and time 
and the results are being correlated with properties of the resulting magnetic thin films.

- The previously developed thermodynamic model for the prediction of slagrefractory chemical interaction was further expanded. The model database currently contains over 200 components, including many complex oxide liquids, and includes compounds of over 19 different elements. Significant additions from the latest JANAF tables have been incorporated on a test basis, prior to making this database compatible with other available databases. Several industries and universities are beginning to substitute this model for the overly simplistic chemical models currently used in transport-based processing computer codes.

- Mass spectrometric and complementary thermochemical investigations were carried out for the ternary $\mathrm{Nb}-\mathrm{Al}-\mathrm{O}$ system, including intermetallic binaries. This work was performed as part of a cooperative DARPA program with CEBELCOR (M. Pourbaix, Belgium) and the University of Florida, for development of oxidation models of aerospace superalloys. Activity data on $\mathrm{Nb}-\mathrm{Al}$ intermetallics and their oxidation products were obtained as part of this study. Data obtained for $\mathrm{NbO}_{2}$ are considered to be more reliable than the existing literature.

- The accuracy of the NIST developed technique of transpiration mass spectrometry was improved through systematic analysis of the system variables using $\mathrm{KCl}$ and $\mathrm{MgCl}_{2}$ as test cases.

- A PC based program and the graphics database for Vols. 6-8 of Phase Diagrams for Ceramists (including nearly 3000 diagrams) was completed and released by NIST's Office of Standard Reference Data for commercial use.

- A three volume conference Proceedings was published. The manuscripts were presented as part of an IUPAC-sponsored, NIST-organized and hosted, international conference on High Temperature Materials Chemistry. Areas covered include (1) Advances in Measurement Techniques, (2)

Thermochemistry and Models, (3) Processing and Synthesis, and (4) Performance under Extreme Environments. Ninety-eight comprehensive papers make up the Proceedings, which will be the first reference work covering the multidisciplinary area of materials chemistry at high temperatures in such depth.

Thermodynamic and Kinetic Stability of Refractory Materials at Ultra-High Temperatures

D. W. Bonnell, A. J. Paul, P. K. Schenck, J. W. Hastie

Coupling laser heating with mass spectrometric and optical spectroscopic analysis has the potential for providing quantitative thermochemical data for refractory materials at temperature and pressure extremes previously inaccessible by conventional techniques. These data are needed for design of high performance materials, e.g., for hypersonic transport vehicles, defense applications, and nuclear power generation. In addition, degradation of 
materials by high powered lasers is important in, for example, the design of laser fusion processes, laser welding, laser processing of ceramics (most recently, superconducting and diamond films), laser etching of semiconductor components, laser annealing of surface alloys, and in the durability of refractories in defense and space applications.

A focused Nd/YAG laser system has been used to vaporize refractory materials and mass spectrometric analysis indicates that the vapor plume beam sampling procedure generally produces negligible post-vaporization perturbation of the neutral species identities and concentrations. The Laser Vaporization Mass Spectrometric (LVMS) technique utilizes time-resolved mass analysis to provide time-of-flight, species specific, information on temperature, ionic and neutral precursors, and the time history of the laser heating process. Both mass and optical spectrometric time-of-arrival data indicate that ions directly produced by the laser heating process are not at thermal equilibrium with the neutral atomic and molecular vapor species. A major objective of current work is to relate the results obtained by mass spectroscopy, which detects thermal neutrals, with optical spectroscopic results, which are sensitive only to ions and highly excited species. Systems currently under investigation include the refractory carbides ( $\mathrm{Ta}_{2} \mathrm{C}, \mathrm{SiC}$ ), $\mathrm{MgO}$, and the most refractory known oxide, $\mathrm{HfO}_{2}$.

\section{Laser Deposition of Electronic Thin Films}

P. K. Schenck, D. W. Bonnell, J. W. Hastie, A. J. Paul, L. P. Cook , C. K. Chiang*

*Electronic Materials Group, Ceramics Division

The objective of this project is to develop a state-of-the-art laser thin film deposition facility for the determination of mechanisms of thin film formation and for the production of thin films for electronic device development. Time resolved mass spectra were obtained using the LVMS technique for many neutral and ionic species in the laser generated plume from high $\mathrm{T}_{c} \mathrm{YBa}_{2} \mathrm{Cu}_{3} \mathrm{O}_{\mathrm{z}}$ targets. $A$ variety of ceramic materials including $\mathrm{MgO}, \mathrm{BaTiO}_{3}, \mathrm{Al}_{2} \mathrm{O}_{3}$, yttria/zirconia, mullite and $\mathrm{Bi}_{2} \mathrm{Sr}_{2} \mathrm{CaCu}_{2} \mathrm{O}_{\mathrm{x}}$ were also deposited as thin films using Nd/YAG laser vaporization.

An Excimer laser system was recently installed to allow vaporization of targets using UV laser light. $\mathrm{Bi}_{2} \mathrm{Sr}_{2} \mathrm{CaCu}_{2} \mathrm{O}_{x}$ thin films, deposited using $193 \mathrm{~nm}$ excimer light from the new laser system, were found to be superconducting $\left(\mathrm{T}_{\mathrm{c}}=80 \mathrm{~K}\right)$ after annealing $\left(810-850^{\circ} \mathrm{C}, 24-72\right.$ hours in air).

In cooperation with the US Army Harry Diamond Laboratories (HDL), laser deposition studies of lead zirconate titanate (PZT) thin films on silicon started in FY89 have continued. A high speed optical multichannel analyzer was used to monitor emission spectra of the laser generated plumes from PZT targets. Analysis of these spectra indicates that the laser pulse forms a plasma, containing ionized metal atoms, which persists after the laser pulse. This result suggests that the interaction of the plasma with the target surface may, in part, influence the vaporization. Researchers at HDL patterned memory devices on NIST-produced PZT films on silicon. Electrical measurements on the patterned films exhibited ferroelectric hysteresis loops which are the basis of the use of this material in non-volatile memory chips. 
PZT films deposited with the new excimer laser exhibited better morphology and ferroelectric properties than those deposited in our earlier work with the Nd/YAG laser.

\section{Laser Deposition of Magnetic Thin Films}

J. W. Hastie, P. K. Schenck, D. W. Bonnell, A. J. Paul, R. D. Shul1*, * Magnetic Materials Group, Metallurgy Division

The overall technical approach of this project, initiated in FY91, involves the correlation of the molecular level characteristics of the vapor/plasma present during the deposition process with the structure and properties of magnetic thin films. The process selected for investigation was the pulsed laser deposition of magnetic films containing $\mathrm{Fe}_{3} \mathrm{O}_{4}$ and $\mathrm{Ag}$, for which previous ion sputtering experience suggests a relationship of film properties to deposition chemistry exists.
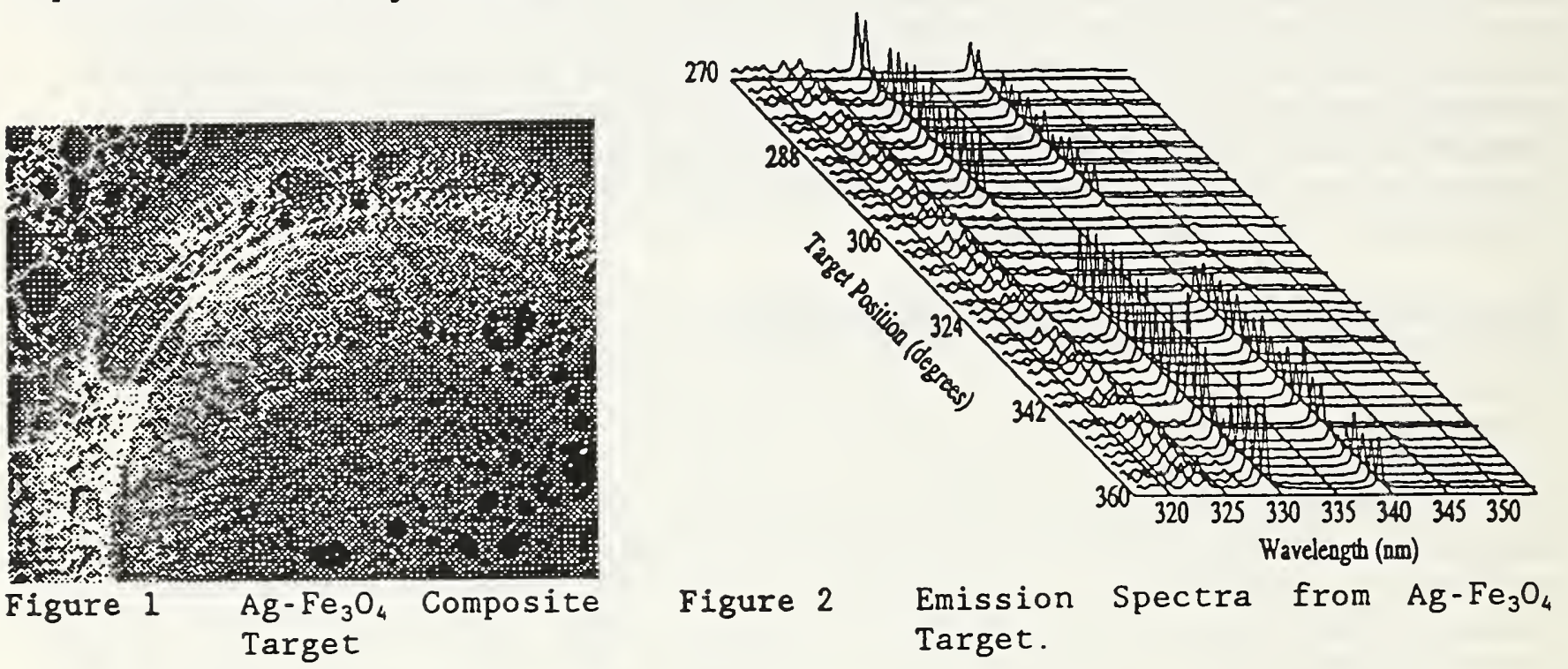

High pressure molecular beam sampling techniques are currently being used to measure the sputter-transferred vapor species as a function of both location and time.The angular distribution of material vaporized in the plumes formed from $\mathrm{Fe}_{3} \mathrm{O}_{4}, \mathrm{Ag}$, and $\mathrm{Ag}-\mathrm{Fe}_{3} \mathrm{O}_{4}$ targets are also being observed using the LVMS technique. Preliminary observations of the data show the distribution of material formed in each of these plumes to differ when using the $1064 \mathrm{~nm}$ beam of a Nd/YAG laser. In addition, the angular distribution of some ions produced in these plumes are different from the neutrals. The plume formed in the $\mathrm{Fe}_{3} \mathrm{O}_{4}$ target is predominantly $\mathrm{Fe}$ with some $\mathrm{FeO}$ present. Atomic Ag is found but no molecular Ag has been observed in the Ag plumes investigated so far. Additional information on species identities and thermal distributions are being obtained as a function of time and location using the OMA technique. A photograph of an $\mathrm{Ag}-\mathrm{Fe}_{3} \mathrm{O}_{4}$ composite target is given in figure 1 . Darker areas on the surface of the target are predominantly $\mathrm{Fe}_{3} \mathrm{O}_{4}$. As a test of the ability to correlate the emission spectra with plume composition, the composite target was rotated and time and spatially resolved ( $-1 \mathrm{~mm}$ above target, $200 \mathrm{nsec}$ after laser) spectra were obtained for each rotation position, as shown in figure 2. The Ag resonance lines at 328 and $338 \mathrm{~nm}$ track the amount of Ag present in the plume. Decreases 
in the intensity of the $\mathrm{Ag}$ lines occur when the islands of predominantly $\mathrm{Fe}_{3} \mathrm{O}_{4}$ are in the laser's path.

\section{Steel Slag -Refractory Thermochemistry}

J. W. Hastie, E. R. Plante, D. W. Bonnell

Modeling and complementary experimental work have continued on the development of a generic predictive model of iron and steel-making slag and refractory thermochemistry. In particular, the high FeO-content systems under current study were chosen to meet the needs of the American Iron and Steel Institute/Department of Energy (AISI-DOE) national program to develop a new direct-reduction steel making process.

The model database currently contains over 200 components covering more than 19 elements. This database is being distributed gratis to interested academic and industry groups. The current form of the database is in the form of coefficients yielding $\Delta_{f} G(T)$ for use with the widely available SOLGASMIX code. Recent interest in thermochemistry codes has resulted in a variety of database formats being considered. As the usefulness of thermochemical databases depends strongly on their format, current efforts are directed toward evaluating commercial codes with a view toward more general compatibility. Regular evaluation of the literature continues, with new species of interest to steel-making being added as quality data are found and validated.

\section{Processing and Protection of High Temperature Structural Materials}

J. W. Hastie, E. R. Plante, D. W. Bonnell

This activity represents a collaborative effort among NIST, the University of Florida/Gainesville, and CEBELCOR (Belgium) under a DARPA sponsored program. An industrial panel advises the program. The purpose of this effort is to combine existing data with new experimental determinations and modeling formalisms to develop data-banked information and models that provide for improved design and evaluation of metallic and composite refractory systems. In particular, niobium alloys and the $\mathrm{Nb}-\mathrm{Al}-\mathrm{O}$ system are of interest, with data on mechanisms of oxidation, species transport, thermochemical stabilities, activities, vapor phase interactions, solubilities, and diffusivities being considered important to the development of reliable models of coating/substrate/gas interactions.

Recent Knudsen effusion mass spectrometric measurements have been made on the total evaporation of $\mathrm{Al}$ from $\mathrm{Nb}-\mathrm{Al}$ intermetallics with starting compositions of $\mathrm{Nb}_{3} \mathrm{Al}$ and $\mathrm{NbAl}_{3}$. Time dependent $\mathrm{Al}(\mathrm{g})$ pressures were observed, due in part to the expected decreasing activity of $\mathrm{Al}$ in the intermetallics, but also apparently due to the rate of vaporization being controlled by the rate of diffusion of $\mathrm{Al}$ through the alloy. In experiments where the sample was contained in an alumina $\left(\mathrm{Al}_{2} \mathrm{O}_{3}\right)$ cup, within a tungsten Knudsen cell, the observation of the $\mathrm{Al}_{2} \mathrm{O}^{+}$ion, indicative of $\mathrm{Al}_{2} \mathrm{O}_{3}$ vaporization, showed that the sample-cell reaction:

$$
4 \mathrm{Al}(\mathrm{g})+\mathrm{Al}_{2} \mathrm{O}_{3}(\mathrm{~s})=3 \mathrm{Al}_{2} \mathrm{O}(\mathrm{g})
$$


was approximately at equilibrium. Without the alumina cup, only small initial $\mathrm{Al}_{2} \mathrm{O}^{+}$signals were observed, which quickly disappeared as a small oxide impurity was vaporized. The time dependent decay of the $\mathrm{Al}^{+}$ion signal clearly indicated a non-equilibrium process controlling Al transport.

Evaluation of the literature shows that, in general, some limitation on the vapor transport of $\mathrm{Al}$ from $\mathrm{Nb}-\mathrm{Al}$ alloys results in lower than predicted $\mathrm{Al}$ pressures. This limitation is likely to be due, at least in part, to diffusion limitations. Although, in principle, time dependent vaporization data can be used to derive diffusion rates, the observed sintering process has to be accounted for, as well. Knowing the rate of transport of $\mathrm{Al}$ from $\mathrm{Nb}-\mathrm{Al}$ alloys is required to reliably predict the rate of formation and extent of protective oxide layers for $\mathrm{Nb}-\mathrm{Al}$ alloys.

\section{Transpiration Mass Spectrometry of Metal Chlorides}

J.W. Hastie, D.W. Bonnell, and E. Rudnyii*

*Guest Scientist - Moscow State University

The transpiration mass spectrometric (TMS) technique, developed earlier in our laboratory, is in routine use, worldwide, for the molecular level analysis of high temperature vapors at pressures to atmospheric. Current work focusses on improving the accuracy of the method. Certain metal halide systems are relatively simple in their vaporization behavior and considerable non-TMS literature data exists for comparison with the TMS method.

Existing literature data for the $\mathrm{MgCl}_{2}$ and $\mathrm{KCl}$ systems include non-molecularspecific vaporization rates obtained by Knudsen and torsion-effusion weightloss methods, classical transpiration methods, and total vapor pressure data. In addition, molecular-specific Knudsen effusion mass spectrometry data and molecular constants of selected gaseous species are available. Mass spectrometry shows the vapor over $\mathrm{KCl}$ and $\mathrm{MgCl}_{2}$ to consist primarily of monomer and dimer molecules. A major limitation in assessing all the available data is the determination of the relative partial pressures of monomer and dimer. Traditional mass spectrometric measurements require correct partitioning of fragmentation pathways and accurate electron impact cross section data to convert ion intensity data to partial pressures. The TMS technique, operating at higher total and partial pressures than classical mass spectrometry methods allows for measurements at different temperatures, and with non-thermal internal energies, thus giving a more direct handle on fragmentation pathways. In addition, direct determination of relative cross sections is possible, and determination of the importance of temperature dependent fragmentation can be made. Using a modification of the classical double oven technique, the TMS system can isolate gas-gas from gas-condensed reactions, providing further information on true species concentrations and fragmentation pathways. In these systems, the ions $\mathrm{K}_{2} \mathrm{Cl}^{+}$and $\mathrm{Mg}_{2} \mathrm{Cl}_{3}{ }^{+}$are formed unambiguously from the dimer molecules. However, the ions containing only one metal atom can arise from both monomer and dimer molecules. 


\section{Phase Diagram Graphics}

P. K. Schenck

Computer software has been developed to handle complex binary and ternary phase diagrams using stand alone desk top computers in support of the Phase Diagrams for Ceramists Data Center. This graphics system generated the publication-ready diagrams for the recently published (American Ceramics Society) Vols. 6 - 8 of Phase Diagrams for Ceramists. The software has been modified so that the individual work stations in the Data Center can access any diagram from a central mass storage system via the shared resource management system (local network). This software also was used to digitize the diagrams for both an annual and high $T_{c}$ superconductor supplements of Phase Diagrams for Ceramists. Software also was developed to provide PCaccess to the ceramics phase diagram graphics database. PC-users will have the ability to retrieve data from the screen display in a choice of user units (eg. ${ }^{\circ} \mathrm{C},{ }^{\circ} \mathrm{F}$, or $\mathrm{K}$ ). Mixture compositions in binary phase fields can be determined interactively by use of the lever rule and the results displayed on the PC-monitor screen. The PC based program and the graphics database for Vols. 6-8 (including nearly 3000 diagrams) are scheduled (Fall, 1991) to be released in conjunction with a bibliographic database by NIST's Office of Standard Reference Data. 

The Magnetic Materials Group: studies advanced magnetic and superconducting materials to characterize their properties and performance, and promotes their economical processing and efficient use; develops and maintains competence in ( 1 ) metallurgical, magnetic, and electronic structure of materials and their correlation with processing conditions, (2) magnetic measurement methods, (3) magnetic reference standards, and (4) magnetic techniques of nondestructive evaluation of materials and structures; and provides expertise to industry, universities, standards organizations, and government agencies.

Magnetic materials are important to the commerce of the nation. The world market for magnetic technology in information storage and electrical equipment (e.g., motors and power transformers) exceeds $\$ 83$ billion/year. Magnetic nondestructive evaluation methods are used for quality control wherever steel is used. Conventional superconductors have many commercial uses, including for medical magnetic resonance imaging [MRI] technology, and the new high-temperature superconductors have important potential applications, both near-term and future.

\section{FY 91 Significant Accomplishments}

- Developed, produced, and certified magnetic susceptibility samples for the testing, alignment, and calibration of mine detectors during Operation Desert Storm.

- Installed and calibrated a ferromagnetic resonance (FMR) apparatus. Measurements were made on both multilayered structures and granular nanocomposites. The angular dependence of the FMR signal was used to determine their magnetic anisotropy.

- Developed theoretical expressions for magnetocaloric effects in superparamagnetic and super-ferromagnetic nanocomposites, including saturation effects. Designed and ordered a new magnet/calorimeter for magnetocaloric measurements.

- Produced a bulk YBCO high $\mathrm{T}_{C}$ superconductor with zero Meissner effect and a significant reduction in giant flux creep due to very strong flux pinning.

- Investigated flux pinning in very high purity YBCO single crystals, before and after detwinning. For such crystals at low fields, the twins contribute significantly to flux pinning. At high fields, the pinning is about the same with or without the twins.

- Prepared bulk nanocomposites of $\mathrm{Fe}$ in vitreous alumina gel. The magnetic properties were in sharp contrast to previously prepared Fetsilica gel: the $\mathrm{Fe}$ magnetic moment in the alumina gel was smaller and varied in the opposite sense with the Fe content. 
Improved the dynamic range of our Barkhausen apparatus, and obtained significant correlation between the Barkhausen signal and the mechanical properties of sheet steel.

Upgraded the vibrating sample magnetometer (VSM) to provide an automated sample rotation capability, and to permit measurement of both longitudinal and transverse components of the magnetization. This vector VSM was used to measure the magnetic anisotropy of $\mathrm{fcc}$ Co in a single crystal Cu-Co multilayer.

Measured the initial magnetization process in magnetic recording tapes and compared with the a Preisach model of magnetic hysteresis.

Reported to DOC on high-magnetic-field facilities in Japan related to superconductivity.

Completed construction of the Magneto-Optical Kerr Effect (MOKE) laboratory.

\section{High-Temperature Superconductors}

L. J. Swartzendruber, L. H. Bennett, H. J. Brown, R. V. Drew

Some very high quality single crystals of $\mathrm{YBa}_{2} \mathrm{Cu}_{3} \mathrm{O}_{7-x}$, produced in the Ceramics Division, have made it possible to observe flux pinning effects due to twin boundaries which had not been previously seen. These effects were observed by two sets of measurements: (1) measurement of the trapped flux as a function of temperature (thermoremanence) displayed in Fig. 1, showed clearly that, for low applied magnetic fields, twins contribute significantly to flux pinning over the temperature range $60 \mathrm{~K}$ to $85 \mathrm{~K}$ when the vortices are parallel to the twin boundaries (i.e., parallel to the c-axis). Little or no contribution to pinning by the twin boundaries was found when the vortices were perpendicular to the $c$ axis; and (2) by measuring hysteresis loops both before and after twin removal. The hysteresis loops in Fig. 2 show a large difference in the low field hysteresis loops at 77K. These effects have not been previously seen because the small contribution to pinning made by the twins was masked by the larger contributions due to imperfections in the crystals.

We have observed some remarkable magnetic properties in a $\mathrm{Y}_{1} \mathrm{Ba}_{2} \mathrm{Cu}_{3} \mathrm{O}_{7-\delta}$ bulk superconductor prepared in collaboration with the Ceramics Division. A magnetic normal $\rightarrow$ superconducting transition width of $\simeq 0.3 \mathrm{~K}$ was measured on the ac susceptometer. This is unusually narrow for a bulk 123 superconductor and comparable to the best single crystals. Very strong magnetic levitation by attraction also was observed. Hysteresis studies and temperature dependence of magnetization display strong flux pinning even at temperatures very close to $\mathrm{T}_{\mathrm{C}}$. This strong pinning appears to result in a significant reduction in the giant flux creep, which, until now, has presented an important barrier to practical applications. Based on the very wide hysteresis loops observed, a high magnetic critical current density is calculated. The strong flux pinning at high temperatures is seen to result in a zero Meissner effect even for low applied fields (Fig. 3). 
In contrast to the zero Meissner effect which was measured with the vibrating sample magnetometry (VSM) on this highly-pinned superconductor, SQUID Meissner measurements paradoxically showed positive values for the magnetization when it was cooled from above $T_{C}$ in a field of $400 \mathrm{~A} / \mathrm{m}$. We believe that this result, i.e., a positive Meissner effect, is aphysical and is an experimental artifact inherent to the commercial SQUID detection, and we intend to pursue its origin. Since Meissner effect measurements are often used in science and in commerce to characterize high $\mathrm{T}_{C}$ superconductors, the discrepancy observed in the two instruments presents a serious measurement problem.

\section{Compositionally-Modulated-Alloy Thin Films}

L. H. Bennett, L. J. Swartzendruber, R. D. McMichael, R. V. Drew

In a cooperative program with IBM, compositionally-modulated alloys (CMA) with individual layers in the nanometer range, produced by the Electrodeposition Group using electrochemical deposition from a single bath, are being investigated using VSM, SQUID magnetometry, and ferromagnetic resonance (FMR). We earlier demonstrated that magnetic measurements constitute an indispensable and meaningful characterization tool for $\mathrm{Ni} / \mathrm{Cu}$ multilayer thin films. Two new multilayer systems, $\mathrm{Co} / \mathrm{Cr}$ and $\mathrm{Co} / \mathrm{Cu}$, produced by electrochemical deposition are being investigated. The $\mathrm{Co} / \mathrm{Cr}$ system has importance for application to magnetoresistive recording heads.

The Co deposition on the $\mathrm{Cu}$ is highly epitaxial and always fcc. The magnetic properties are similar to those obtained by various evaporative methods. In contrast to $\mathrm{Ni} / \mathrm{Cu}$, the phase diagram of Co-Cu permits little alloying. Thus, the saturation magnetic moment per Co atom is almost the same as in bulk Co and only slightly dependent on interface diffusion. A magnetic aftereffect also is evident in these films. As in $\mathrm{Ni} / \mathrm{Cu}$, when deposited on a single crystal substrate, the multilayer shows a strong tendency to $z$-coherence; in addition $x-y$ coherence is evident from FMR (Fig. 4) and vector magnetization measurements (Fig. 5) showing 4-fold anisotropy in the multilayer plane. The effective value of the uniaxial anisotropy field, $\mathrm{H}_{u}$, estimated by comparing in-plane and out-ofplane hysteresis loops is close to $4 \pi \mathrm{M}_{\mathrm{S}}$ for $\mathrm{Co}$.

\section{Nanocomposite Materials}

R. D. Shull, R. D. McMichael, I. J. Swartzendruber, and I. H. Bennett

Composite materials (immiscible metals and oxides with a size scale of the order of nanometers) have been shown to possess unusual composition-dependent electronic and magnetic properties and permit the atomic engineering of specific material properties. Previously thin film $\mathrm{Ag}+\mathrm{Fe}_{3} \mathrm{O}_{4}$ nanocomposites had been prepared by cosputtering and the properties were found to be highly dependent on the method of preparation. This year, a project was started, in collaboration with the High Temperature Materials Chemistry Group, for the preparation of similar thin-film nanocomposites by laser ablation. Composite targets of Ag and magnetite $\left(\mathrm{Fe}_{3} \mathrm{O}_{4}\right)$ were prepared and the plasmas created by their laser ablation were analyzed by optical mass spectrometry. Correlations between the properties of the thin films subsequently deposited and the vapor species are being developed in an attempt to understand the precursor processes occurring in the vapor. 
In collaboration with the Ceramics Division, bulk nanocomposite materials were prepared from homogeneous gelled composites of iron and vitreous alumina containing 10-40 percent $\mathrm{Fe}$ by the room temperature polymerization of aqueous aluminum alkoxide solutions containing ferric nitrate and nitric acid at low $\mathrm{pH}$. Scanning electron microscopy, x-ray diffraction, and Mössbauer spectroscopy demonstrated that this bulk material is comprised of nanometer-sized regions of iron compounds embedded in a vitreous alumina gel matrix. Magnetization data showed that in the as-cured condition these nanocomposites are paramagnetic at room temperature and become either superparamagnetic or ferromagnetic on cooling to 10K. The magnetic susceptibility increased with the Fe content and with decreasing temperature. Analysis of the temperature dependence of the magnetic susceptibility indicated the magnetic moment per $\mathrm{Fe}$ atom was $1.87 \mu_{\mathrm{B}}$ for the $10 \%$ Fe nanocomposite (Fig. 6) and that it increased linearly with composition to $1.96 \mu_{B}$ for the $40 \% \mathrm{Fe}$ material (Fig. 7). This is in contrast to the results obtained earlier on Fe+silica gel nanocomposites where the magnetic moment per iron atom was much larger and which decreased with increasing Fe content. This result shows the matrix gel does not just provide a mechanical support for the finely dispersed magnetic species, but also plays an important role in determining the magnetic character of the material. Mössbauer effect data showed that subsequent treatment of these materials in a gaseous environment of hydrogen at low temperatures $\left(\mathrm{T}<400^{\circ} \mathrm{C}\right)$ changed the form of the iron in the magnetic regions.

Bulk nanocomposites of immiscible metals were also prepared by another chemical path, namely coprecipitation from aqueous solutions. Using this route, nanoparticles of copper-iron and copper-cobalt mixtures were produced by coprecipitation from an aqueous solution of their salts and subsequent reduction at temperatures from 125 to $350{ }^{\circ} \mathrm{C}$ in an atmosphere of $\mathrm{H}_{2}$ gas. X-ray diffraction reveals the fcc structure of $\mathrm{Cu}$, and Brunauer, Emmet and Teller (BET) analysis shows that mean particle sizes range from 20 to $90 \mathrm{~nm}$. The magnetic characteristics of these materials were found to depend sensitively on preparation conditions. For instance, $\mathrm{Cu}-\mathrm{Fe}$ prepared using sulfates possessed a much lower susceptibility than similar sulfate free material. Cu-Co prepared in air is quickly reduced at $125^{\circ} \mathrm{C}$ in $\mathrm{H}_{2}(1 \mathrm{hr}$ ) to a form which is nearly stable, but has a magnetization of only $6.5 \mathrm{emu} / \mathrm{g}$, a value 40 percent of that expected for large particles of Co. In contrast, Cu-Co prepared under an atmosphere of dry $\mathrm{N}_{2}$ possessed an even lower magnetization of $2.4 \mathrm{Am}^{2} / \mathrm{kg}$ after the one hour $125{ }^{\circ} \mathrm{C}$ treatment in $\mathrm{H}_{2}$. Further treatment for up to 10 hours at 125 ${ }^{\circ} \mathrm{C}$ in $\mathrm{H}_{2}$ increased the magnetization of this latter material to $10.5 \mathrm{Am}^{2} / \mathrm{kg}$.

\section{Magnetic Refrigeration}

R. D. Shull, L. J. Swartzendruber, R. D. McMichael, and L. H. Bennett

Work on the magnetocaloric effect and magnetic refrigeration has been progressing along both experimental and theoretical paths. Theoretical work included analytical and computational calculations of the magnetocaloric effect in nanocomposite materials. For non-interacting particles expressions were derived which describe an optimum particle size for maximum magnetocaloric effect for any given temperature and applied magnetic field (Fig. 8). Monte Carlo calculations carried out on the Cray supercomputer and mean-field theory calculations also were used to calculate the magnetocaloric effect for ferromagnetically- 
interacting particles. These results show that an assembly of interacting clusters yields an enhanced effect over that for a system comprised of single spins at temperatures higher than the Curie point of the material (see Fig. 9).

Experimentally, a technique was developed for the determination of field-induced entropy changes through magnetization measurements. This technique allows the measurement of small samples relatively quickly and avoids many of the subtleties of conventional calorimetry. Screened candidate materials include metallurgically prepared precipitates of $\mathrm{Co}$ in $\mathrm{Cu}$, rapidly solidified CuCo alloys, Fetsilica gel nanocomposites, chemically coprecipitated Cu+Co nanoparticles, paramagnetic gadolinium gallium garnet (GGG), and a $\mathrm{Zn}$-Co ferrite having pseudo-superparamagnetic behavior.

To make direct magnetocaloric measurements, a magnet/calorimeter system has been designed and ordered. This system includes a 7 tesla, large bore (7.5 cm I.D) superconducting magnet combined with special magnet bucking coils to reduce the magnetic field to zero at a position $30.5 \mathrm{~cm}$ above the maximum field location. Very good vertical thermal homogeneity in the specimen chamber between the maximum and zero-field positions is accomplished through the incorporation of a surrounding high thermal-conductivity copper shield. The temperature of this shielded region may also be controlled between $4.2 \mathrm{~K}$ and room temperature. In addition, a separate insert will allow measurements between $300 \mathrm{~K}$ and $400 \mathrm{~K}$. Operation of the system is expected in early FY92.

\section{Modeling of Magnetic Recording}

L. J. Swartzendruber, L. H. Bennett, M. Pardavi-Horvath*, and F. Z. Vajda* *Guest Scientist - The George Washington University

Frequently, knowledge of the fully demagnetized state of a magnetic material as a well-defined internal magnetic state is required in magnetic applications, modeling, and in magnetic measurements. The question of the existence of a unique demagnetized state is made manifest by the appearance of several demagnetized states at zero field, each having zero magnetization, for an epitaxial single crystal garnet film as viewed in polarized light (Fig. 10). State la was obtained by low frequency ac demagnetization; state $1 b$ as a result of simply turning off the field; lc after application of a saturating field normal to the easy direction, and, $1 d$, the domain structure after thermal demagnetization, i.e. cooling in zero field from above Curie temperature. Although all of these states have a zero net magnetization, only one of them is the equilibrium ground state.

The recently described vector moving model of magnetic hysteresis of particulate recording media is an extension of the classical scalar Preisach model, where the magnetic response of each point of the Preisach plane is generalized to include vector behavior. Thermal demagnetization is simulated by the vector model by randomly orienting the magnetization of the basic particles with the only fields they experience being the local interaction field. The Preisach model predicts distinctly different ac, dc and thermally demagnetized states. The shapes of the virgin magnetization curves predicted by the vector moving model are such that the curve corresponding to ac demagnetization always lies below the curve due to thermal demagnetization, with the curve due to the dc demagnetization lying between them. Of the three demagnetized states, the ac demagnetized state has 
a smaller fraction of magnetizations initially oriented favorably in the direction of the magnetizing field. More energy is therefore required in the former case to rotate the magnetizations into more favorable directions.

The virgin magnetization curves of various magnetic recording media have been measured, after ac and dc demagnetization, with the vibrating sample magnetometer. Samples for the measurements have been cut from commercial recording media: Co-modified $\tau-\mathrm{Fe}_{2} \mathrm{O}_{3}$ and $\mathrm{CrO}_{2}$ tapes and a $\tau-\mathrm{Fe}_{2} \mathrm{O}_{3}$ floppy disk. AC demagnetization along tape axis was achieved with a commercial ac tape demagnetizer device. DC demagnetization was realized after application of a magnetic field sufficient to reduce the remanence to zero in $\mathrm{H}=0$. Thermal demagnetization of these samples could not be examined because the material of the substrates is not heat resistant. The measurements were in very good agreement with the predictions of the vector moving model of demagnetized states.

Magnetic Methods for Intelligent Processing and NDE

L. J. Swartzendruber and D. E. Mathews

Magnetic methods for flaw detection in ferritic steels are widely used both during production and for maintenance. Methods for flaw detection, including the magnetic particle method, detect magnetic leakage fields that occur at defects in a magnetized material. On-line measurement of the magnetic properties of steels holds considerable potential for use in intelligent processing. These methods rely on relationships between magnetic properties such as coercive force, and mechanical properties such as yield strength.

For use as an nondestruction evaluation (NDE) tool, our emphasis has been on providing standards and methods for the evaluation of steel parts, primarily by the use of leakage field methods including magnetic particle methods. Despite the heavy industrial usage and the long history of magnetic particle inspection, there remain a number of fundamental questions concerning how to properly apply, and how to improve, the magnetic particle method. During the current fiscal year work has proceeded on the development and testing of a new magnetic particle test ring. A number of these new rings are being produced, tested, and packaged as a standard reference material for sale by the OSRM. Each ring will be certified using the leakage field pattern from the artificial defects machined in the ring. Fig. 11 shows the design of the ring and the measured leakage field gradients from the defects. In addition, a number of new specifications are being developed in cooperation with the ASTM for improving magnetic particle testing procedures. This work has been partially funded by the Army and includes the preparation and coordination of military standards in cooperation with the Amerocan Society for Testing and Materials (ASTM), the Society of Automotive Engineers (SAE), and various interested industrial organizations.

Because magnetic properties can be measured rapidly, and because they are sensitive to the same microstructural parameters that affect mechanical properties, they have potential for use in the processing control of ferritic steels. Magnetic properties of interest include magnetic Barkhausen noise, magnetoacoustic emission, residual magnetization, hysteresis, permeability, and coercive force. The challenge in using magnetic measurements is to select those measurements, or combination of measurements, which can be determined rapidly in a production environment and which will be effective in determining changes in 
those properties of most interest. During the current year we have improved our capability to measure Barkhausen noise properties by upgrading our 8 -bit digitizer to 12 bit, thereby increasing the upper frequency for measurements from $\approx 25$ to $\approx 50 \mathrm{kHz}$ and we have used this new capability to investigate the relationship of the Barkhausen signal to the mechanical properties of sheet steel. Fig. 12a shows the correlation between a readily measured Barkhausen noise property, the median jumpsum field, and the yield strength for five samples of low carbon sheet steel. Fig. 12b shows the correlation between hardness and yield strength for the same five samples. Both correlations are good except for one sample which is marked HR. This sample was different from the other four in that it had been hot rolled, whereas the others had been cold rolled. The correlation obtained is encouraging and we plan to continue this investigation, with the cooperation of the American Iron and Steel Institute (AISI), by making small processing variations on a single sample of sheet steel. Other magnetic properties of these samples, such as coercive force, will also be measured and methods for measuring magnetic properties on rapidly moving samples will also be investigated.

Assistance was provided to the U.S. Army during Operation Desert Storm. This assistance involved building a special test rig for measurements of low field ac susceptibility and developing, producing, and certifying magnetic susceptibility samples for the testing, alignment, and calibration of mine detectors.

\section{High Magnetic Field Facilities In Japan Related to Superconductivity Research}

R. D. Shull

An evaluation of the high magnetic field facilities in Japan related to superconductivity research was prepared in response to the Japanese Technical Literature Act of 1986 wherein the Secretary of Commerce is required to prepare annual reports regarding important Japanese scientific discoveries and technical innovations in such areas as computers, semiconductors, biotechnology, and robotics and manufacturing. This report (NISTIR 非593, August, 1991) was prepared following a trip to the high magnetic field facilities and superconductivity research centers in Japan, and to the high magnetic field centers in the United States, between August and September 1990.

The highest magnetic field capabilities in Japan are presently comparable to the highest elsewhere in the world, for both $\mathrm{dc}$ and pulsed fields. A concerted effort at the National Research Institute for Metals (NRIM), through the MultiCore Research Project on Superconductivity of the Science and Technology Agency in Japan, is presently being directed toward the development and construction of very high field and greater homogeneity research magnets. The new magnets are projected to be finished in 1993. One of these new magnets would be capable of creating the highest DC field in the world: $40 \mathrm{Tesla}$. The present U.S. limit (currently the highest in the world) is 31.8 Tesla (with 35 Tesla to become available in 1991) at the Francis Bitter National Magnet Laboratory (FBNML). The new U.S. National High Magnetic Field Laboratory, soon to be located at Florida State University (Tallahassee) and at the Los Alamos National Laboratory plans to have a DC field capability of 35 Tesla by 1995 and a 45 Tesla magnet somewhat later. Other centers of high magnetic field expertise in Japan exist at Tohoku University (High Field Laboratory for Superconducting Materials, HFLSM), Osaka University (Research Center for Extreme Materials, RCEM), and the University of 
Tokyo (Institute for Solid State Physics, ISSP). At these locations numerous developments in magnet technology (e.g. $\mathrm{Nb}-\mathrm{Ti}-\mathrm{Hf}$ and $\mathrm{Nb}-\mathrm{Ti}-\mathrm{Ta}$ wires, submicron filamentary wires with enhanced $J_{C}$ values, multifilamentary $\mathrm{Nb}_{3} \mathrm{Al}$ wires, Agsheathed $\mathrm{BiPbSrCaCuO}$ high $\mathrm{T}_{\mathrm{C}}$ tapes, and $\mathrm{Cu} / \mathrm{Al}, \mathrm{Cu} / \mathrm{Ag}, \mathrm{Cu} / \mathrm{Al}_{2} \mathrm{O}_{3}$, and $\mathrm{Cu} / \mathrm{NbTi}$ composites) have been made recently. 


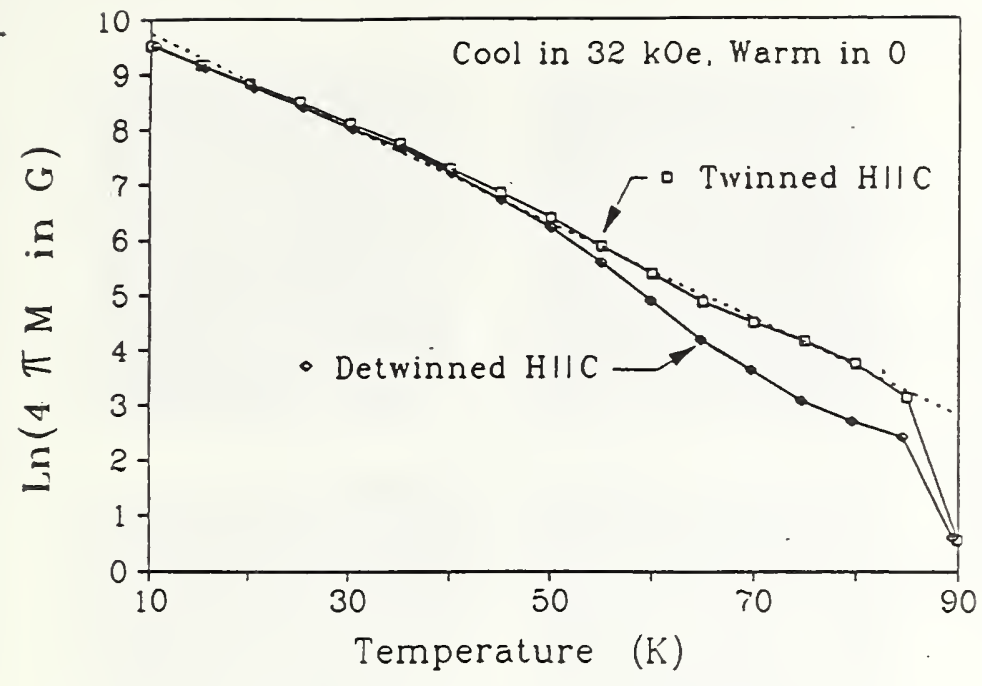

Fig. 1 Thermoremanent magnetization measurements for a high quality $\mathrm{YBCO}$ crystal in the twinned and detwinned conditions. The sample was cooled in a large field to $10 \mathrm{~K}$, the field was removed. The measurements were made on warming.

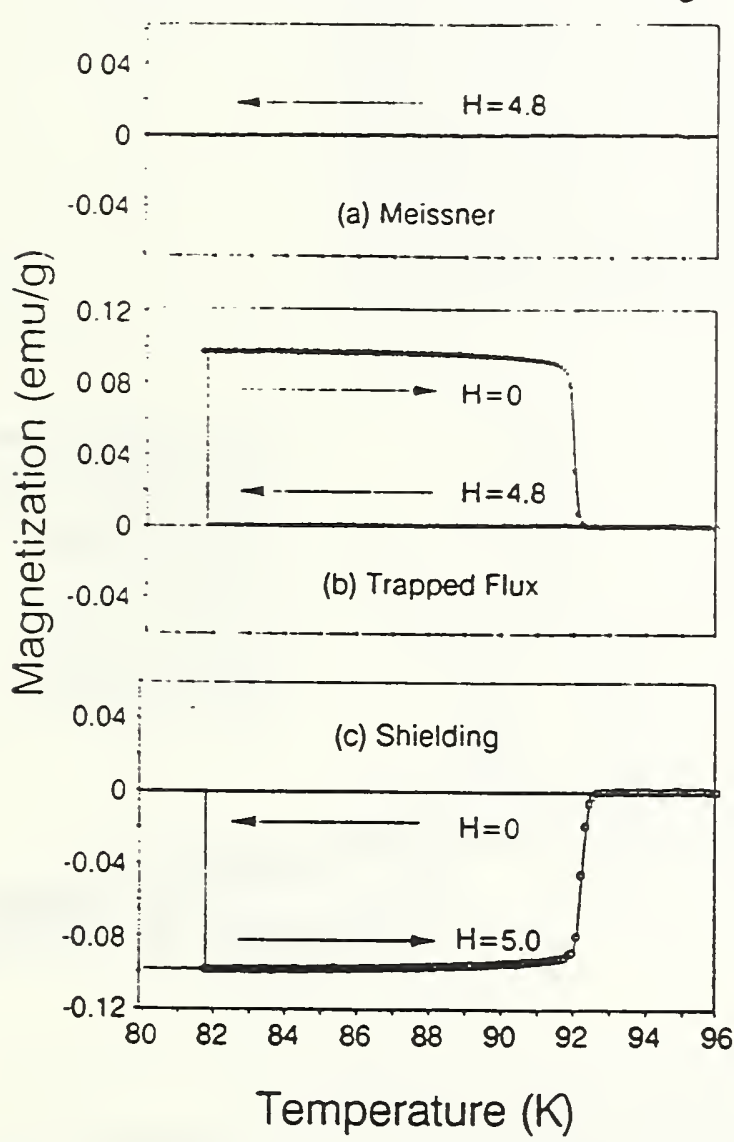

Fig. 3 Magnetization vs. temperature measurements for a highly pinned YBCO superconductor. The arrows show the direction of temperature change. (a) Measurements taken while cooling in a magnetic field from above $T_{c} \cdot$ (b) The field was then turned off, and the magnetization was measured on warming. (c) Cooled from above $T_{c}$ in zero field, and then the magnetization was measured on warming in a $5.0 \mathrm{Oe}$ field.

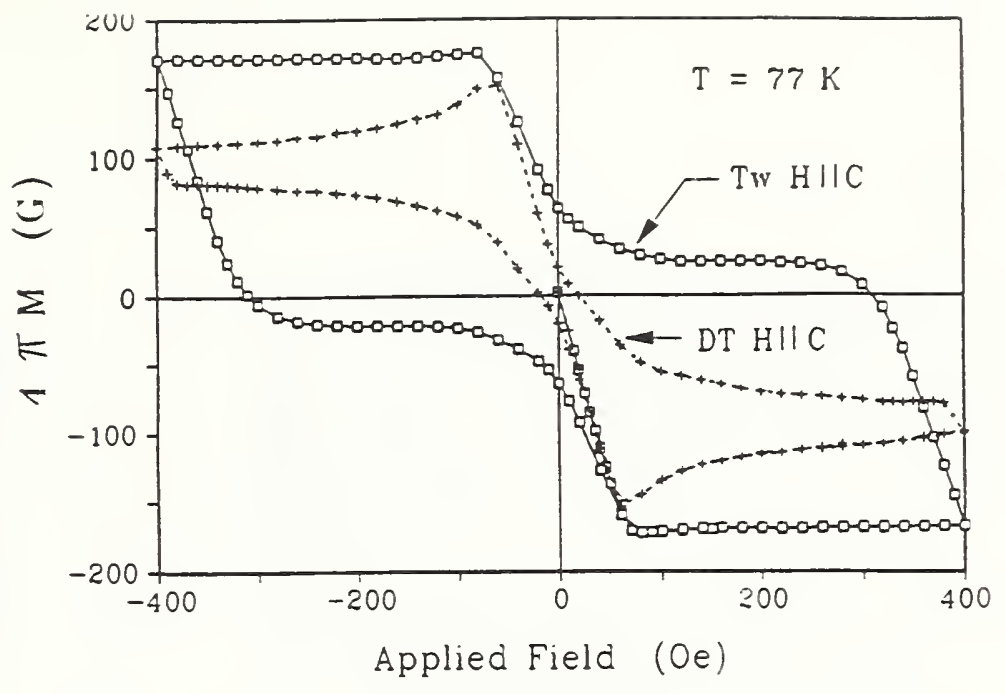

Fig. 2 Hysteresis loops at 77K for the field applied parallel to the $c$-axis of a high quality crystal in the twinned and detwinned conditions. The twinned crystal has significantly greater flux pinning.

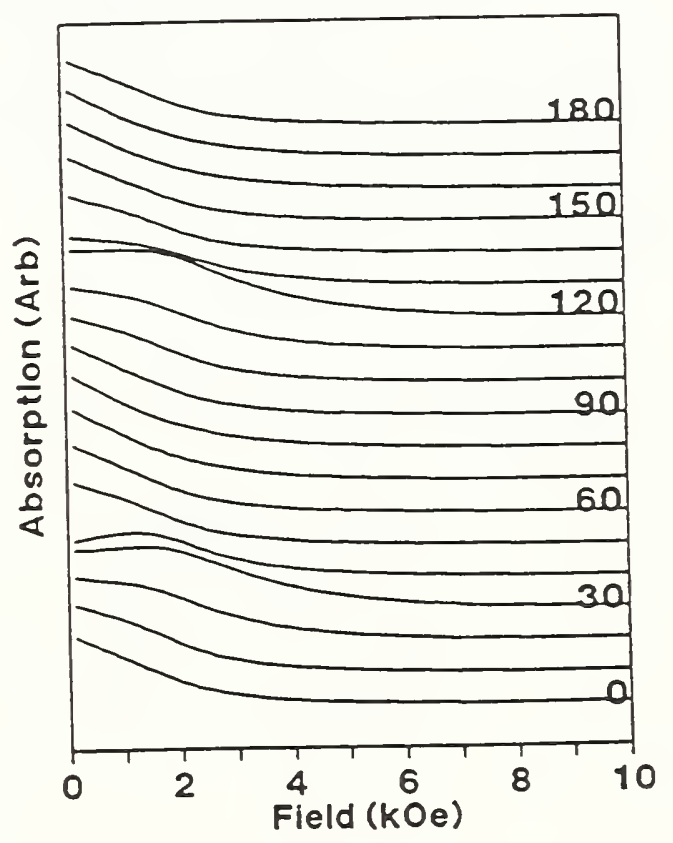

Fig.4 In-plane derivative FMR spectra taken every $10^{\circ}$ from $0^{\circ}$ to $180^{\circ}$ of $\mathrm{Co}-\mathrm{Cu}$ multilayers on $\mathrm{Cu}(100)$ showing fourfold anisotropy.

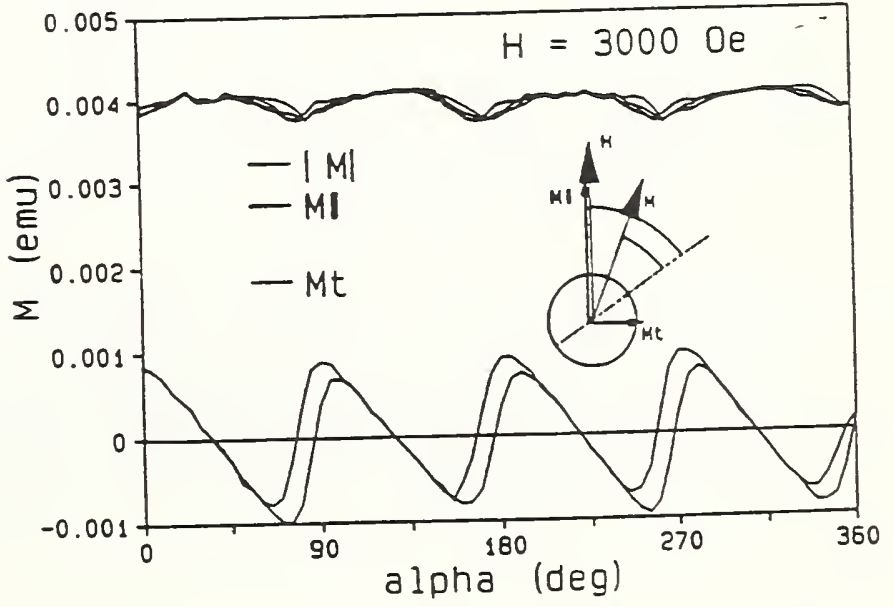

Fig.5 Vector VSM longitudinal and transverse measurements displaying fourfold in-plane anisotropy of Co-Cu multilayers on copper (100). A value of $\mathrm{K}_{1} / \mathrm{M}$ is obtained through analysis of nonhysteretic data in good agreement with published results for fee Co. 


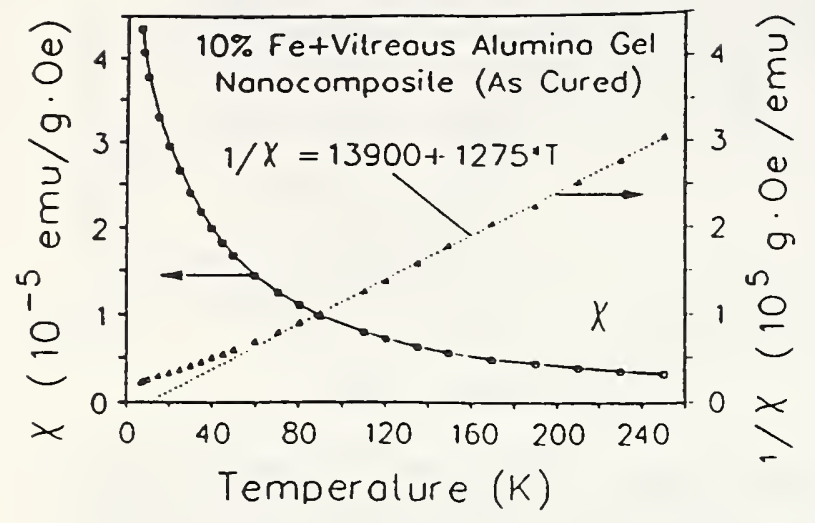

Fig. 6 Magnetic susceptibility ( $\chi$, open symbols) and inverse susceptibility $(1 / \chi$, filled symbols) vs. temperature of the as-cured $10 \% \mathrm{Fe}+$ vitreous alumina gel nanocomposite composite measured at $\mathrm{H}=716 \mathrm{kAlm}$ as the sample was cooled from $300 \mathrm{~K}$. The dashed line is a least squared fit for the $T>70 \mathrm{~K}$ data to a Curie Weiss law.

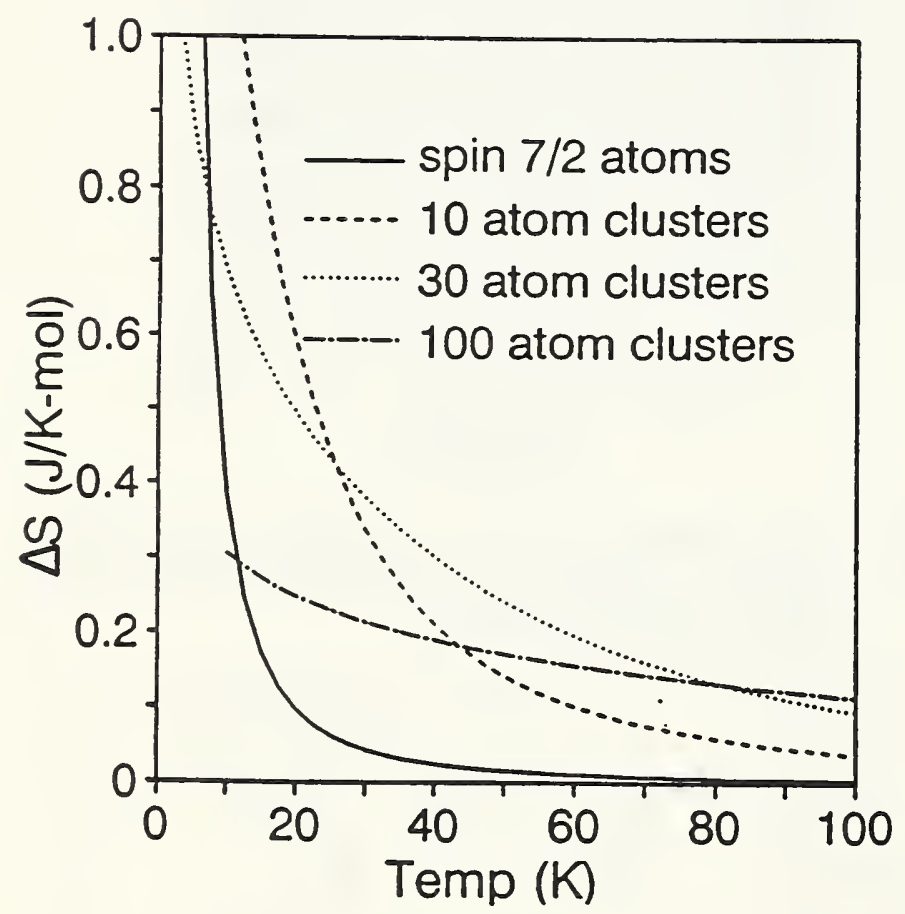

Fig. 8 Entropy change, $\Delta S$, of a superparamagnet with saturation moment $M_{0}$ as a function of particle moment, $m$, induced by removal of a field, $\mathrm{H}$ at a temperature $T$. for a Given value of $H$ and $T$, the maximum entropy change occurs at $\mathrm{mH} / \mathrm{Kt} \simeq 3.5$ where $\mathrm{T} \Delta \mathrm{S} \simeq .272 \mathrm{M} \mathrm{H}$.

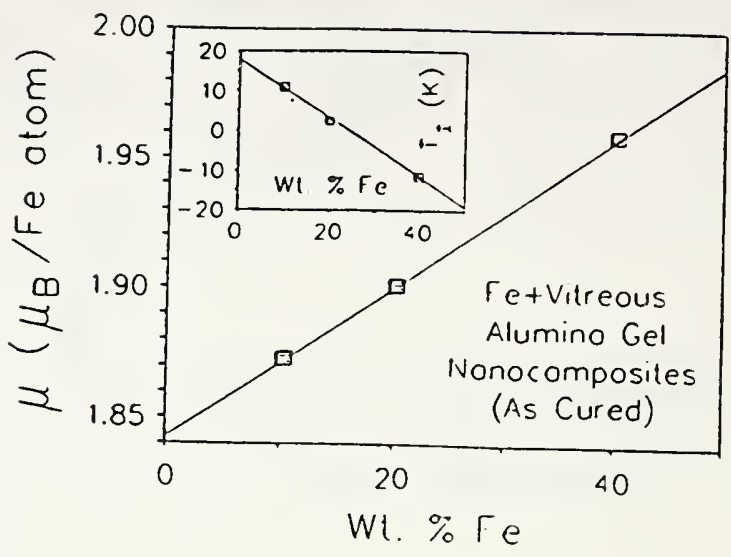

Fig. 7 Magnetic moment per $F e$ atom $(\mu)$ and $T_{1}$ (inset) vs. wt \% Fe in ascured $\mathrm{Fe}+$ vitreous alumina gel nanocomposites determined from $1 / X$ vs. T plots.

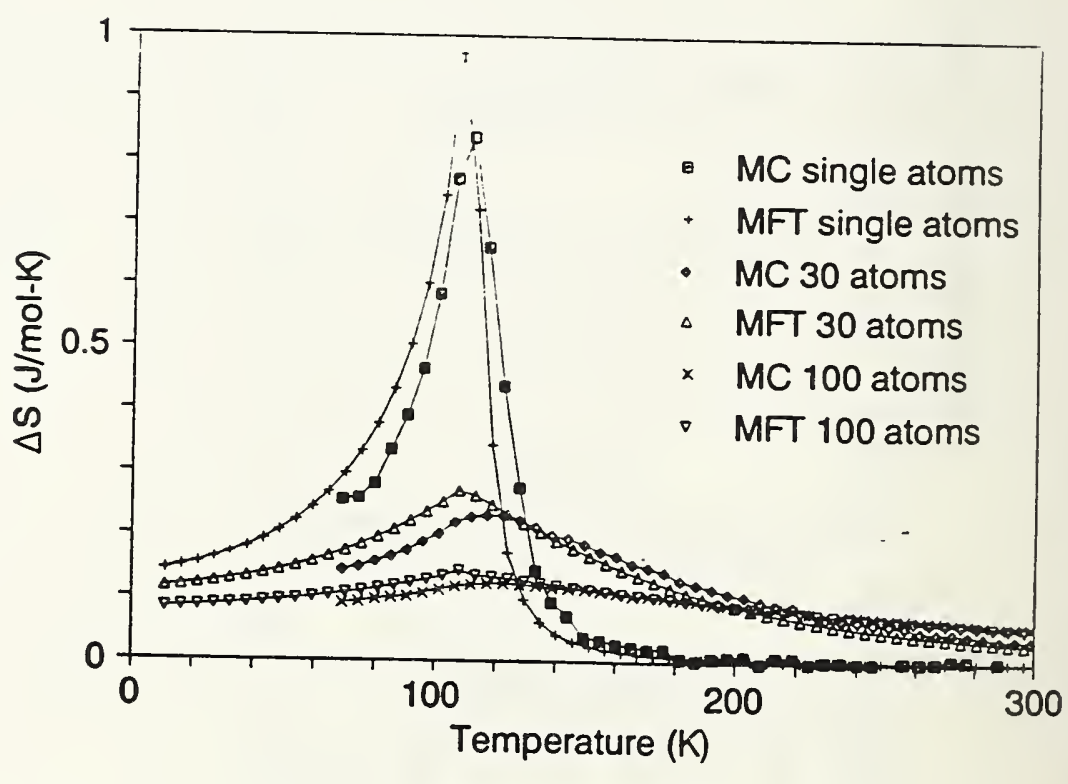

Fig. 9 Entropy change, $\Delta S$, induced by removal of a 1 Tesla magnetic field calculated by both mean-field theory (MFT) and the Monte Carlo method (MC) for a ferromagnetic material, and for interacting superparamagnetic clusters of 30 - and 100 atoms, all having the same ordering temperature of $108 \mathrm{~K}$. 

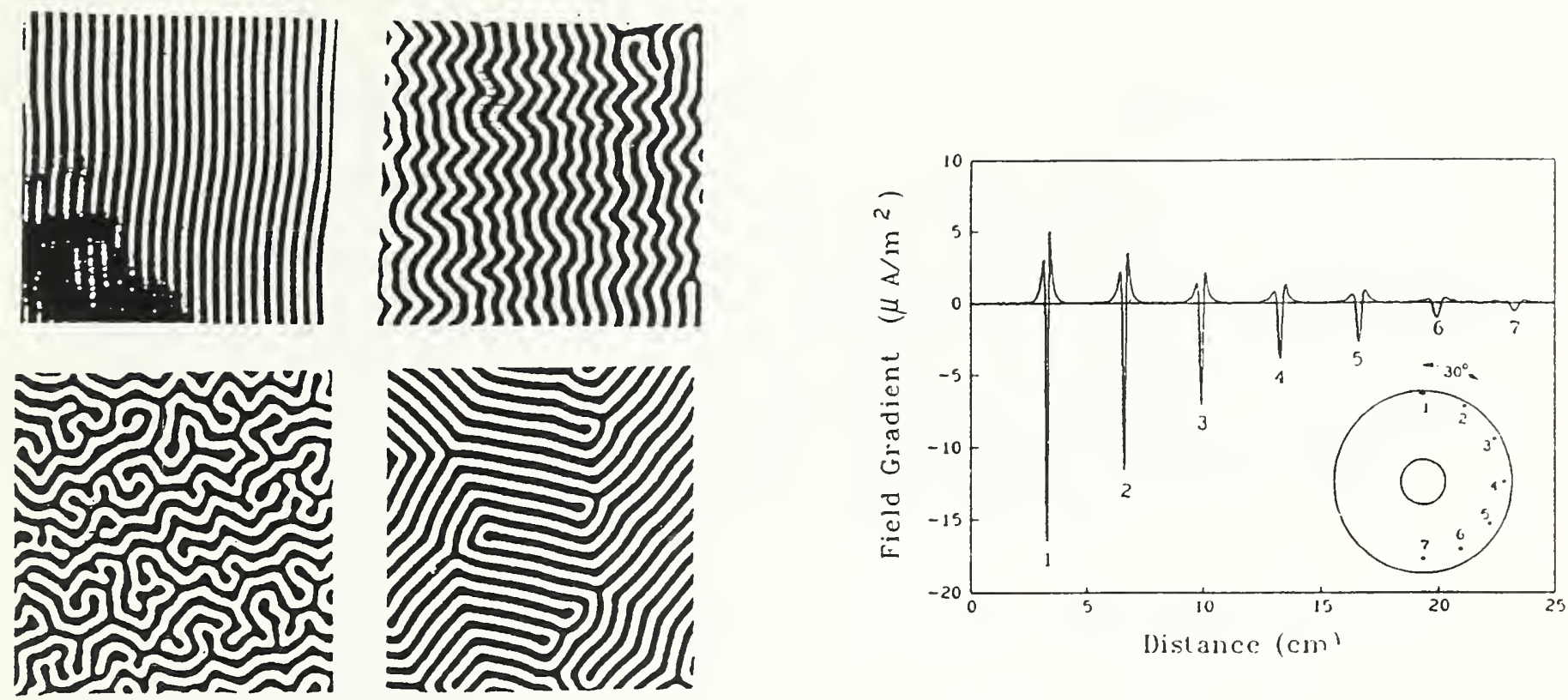

Fig. 10

Different $\mathrm{M}=\mathrm{O}$ states in $\mathrm{H}=\mathrm{O}$ for a single crystalline magnetic garnet film, obtained after a - AC demagnetization, $B$ - the field is switched off, c - application in large field in the hard direction, $d$ - thermal demagnetization.
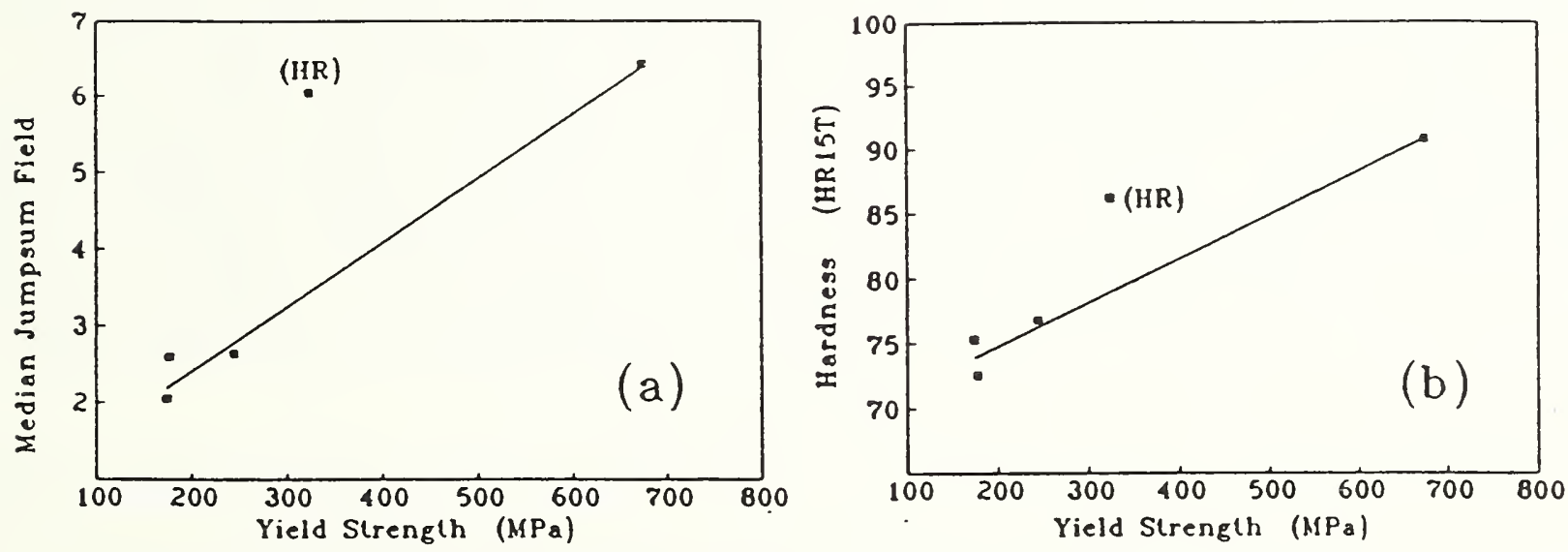

Fig. 12 Correlation of the yield strength measured for five samples of mild steel sheet with (a) the median jumpsum field determined using Barkhausen noise, and (b) the hardness. All samples were in the cold rolled condition except for the sample labeled (HR) which was hot rolled. 

The Mechanical Properties and Performance Group develops new and improved test methods and standards to facilitate accurate measurements of the mechanical characteristics of advanced materials, such as composites and intermetallics, as well as of conventional materials under the kinds of severe environmental and operating conditions that are experienced in processing and in service; and generates reliable mechanical property data and models. The Group also provides expert consultation and testing services relating to the mechanical performance of materials and structural components, including failure

analysis, for other government agencies and standards organizations and, under appropriate conditions, for the private sector as well.

Some noteworthy accomplishments were realized this year; several of the technical accomplishments are summarized below. On the administrative side, Dr. Richard Fields was granted an award by the Harry Diamond Laboratories (HDL) for his research last year on electronic solder connections, and HDL assigned guest scientist Andrew J. Bayba to assist Dr. Roland dewit in this year's work on this project. Recognizing, however, that the Group is nearing the end of only the second year of its existence, perhaps the most significant achievement has been the gradual maturation of its program. What had originated as a disparate collection of inherited tasks has begun to narrow, with a few foci of scientific and engineering interests beginning to emerge as important areas of emphasis for the long term. In this context, some of the projects that were started this year may be more important, programmatically, than some which were successfully concluded.

A sizable portion of this year's research efforts was devoted to the fracture behavior of engineering alloys -. an area of special expertise for the Group - and plans for an international initiative on crack-arrest fracture toughness were formulated. A project on the mechanical properties of metal-matrix composites was initiated and is expected to grow into a substantial programmatic thrust over the next several years. The Group's responsibilities to provide expert consultative and testing services also figure in its strategic plans. The Secretary of Commerce delegated to NIST the task of implementing the Fastener Quality Act of 1990, and the Mechanical Properties and Performance Group undertook a major effort to establish the mechanical testing criteria which will be used to accredit fastener testing laboratories. This is a highly visible project with significant implications for the national economy. It is expected that the Group will be involved in aspects of this project for several years.

\section{FY 91 Significant Accomplishments}

- On the basis of measurements made on the fractured specimens from the NIST wide-plate crack-arrest test program, it has been shown that the thickness reduction along a crack propagation plane can provide a useful estimate of the fracture toughness of the material.

- The growth of copper-tin intermetallic compounds in electronic solder connections was modeled analytically. The model confirms the 
experimental observation that the $\mathrm{Cu}_{6} \mathrm{Sn}_{5}$ intermetallic grows much more rapidly than the $\mathrm{Cu}_{3} \mathrm{Sn}$ intermetallic, and shows that very large stresses are developed around the $\mathrm{Cu}_{6} \mathrm{Sn}_{5}$ intermetallic layer.

Extensive testing programs were carried out on AAR TC128 grade B, which is a widely used tank car steel for the transportation of hazardous materials. It was shown that the impact and fracture toughness properties of the material in the normalized and stress-relieved condition are at least equal to, and generally better than, the corresponding properties in the normalized condition. It also was shown that when the normalized material is made using the new inclusion shape control practice (ISCP), its fracture toughness properties are better than those of an experimental control-rolled steel, A 8XX, also made with ISCP, which had been considered as a potential replacement for AAR TC128 grade B.

In metal powder processing it is usually difficult to achieve near-net shape in the final piece -- particularly if it is an irregularly shaped object - - because the shape changes that occur during consolidation are inadequately understood. Equations were developed this year that enable the consolidation and the plastic shape change of a titanium powder compact to be estimated from the density of the compact and the stress state acting upon it.

- Precision measurements were made of Poisson's ratio in compression for two stainless steels. Because of alignment sensitivities and the exceedingly small strains involved, this is probably the most difficult elastic constant to measure accurately, and has rarely, if ever, been cited in the literature.

Use of Thickness Reduction to Estimate Fracture Toughness Roland deWit, Richard J. Fields, and George R. Irwin* * University of Maryland

To help predict the behavior of a nuclear pressure vessel undergoing pressurized thermal shock, values of the crack-arrest toughness are required. The purpose of the work described here is to show how toughness values can be estimated from the thickness reduction (TR) of tested specimens and then used as "lower-bound" estimates. In the NIST wide-plate crack-arrest test program, sixteen single-edge-notched tensile specimens were fractured using the 26.7-MN universal testing machine. The first series of tests used HSST A533 Grade B Class 1 quenched and tempered steel, while the second series used a low upper-shelf $21 / 4 \mathrm{Cr}-1$ Mo steel. For each specimen the TR was measured on the two halves of the broken specimen and a contour map was constructed. The thickness reduction along the crack propagation plane can be related to the toughness, $\mathrm{K}$, by the relationship $\mathrm{K}^{2}=\mathrm{E} \cdot \sigma_{\mathrm{Y}} \bullet \mathrm{TR}$, where $\mathrm{E}$ is Young's modulus and $\sigma_{Y}$ an estimate of the effective tensile yield strength adjusted for temperature, strain rate, and constraint. The results show good agreement with values of $\mathrm{K}$ for arrest and re-initiation computed from a finite-element generation-mode analysis. Therefore, it appears that the indication of local plastic work rate provided by the thickness reduction near the fracture plane can serve as a useful preliminary assessment of the fracture toughness. 
Crack-Arrest Fracture Toughness of Two Tank Car Steels Used in the Transportation of Hazardous Materials

George E. Hicho

Crack-arrest fracture toughness tests were conducted for the Federal Railroad Administration on normalized AAR TC128 grade B steel and an experimental control-rolled steel, A $8 \mathrm{XX}$, considered as a potential replacement for normalized AAR TC128 grade B steel. Both steels were made to fine grain practice using a new refining process, inclusion shape control practice (ISCP), to control the shape of the sulfide inclusions. Steels made using the conventional process contain manganese sulfide inclusions that are lenticular in shape. ISCP caused the inclusions to become spherical in shape. This reduces the anisotropy in the wrought steel and increases the upper shelf impact energy compared with steels made using normal refining processes. Static fracture toughnesses measured previously showed that the normalized and inclusion shape controlled AAR TC128 grade B steel was more resistant to crack initiation than the new experimental steel A 8XX. Data such as these are often considered conservative because they represent fracture toughness under static loading conditions. More characteristic of the true or lower bound fracture toughness is the crack-arrest toughness. This value is a measure of the steel's ability to arrest a propagating crack. Crack-arrest results for these two steels showed that the normalized and inclusion shape controlled AAR TC128 grade B steel was slightly better than the new experimental steel, A $8 \mathrm{XX}$.

\section{Characterization of a Tank Car Steel Used in the Transportation of Hazardous Materials. George E. Hicho and Donald E. Harne}

Research was conducted for the Federal Railroad Administration on normalized, and normalized and stress relieved AAR TC128 grade B steel. (Unlike the steel described above, this steel was not made using inclusion shape control practice). Traditional mechanical property tests for ultimate and yield strength, reduction in area, elongation, impact, and the nil-ductility temperature were performed on the steel in both of these conditions from -51 ${ }^{\circ} \mathrm{C}$ to room temperature. In addition, the crack initiation fracture toughness, as a function of temperature, was determined using crack tip opening displacement and $J$ integral test procedures. The crack arrest fracture toughness, as a function of temperature, also was determined. Impact results showed that the normalized and stress relieved material has better impact properties at low temperatures and higher upper shelf energy values than the normalized material. Low temperature fracture toughness results also showed that the normalized and stress relieved material is more resistant to crack initiation than the normalized material. The crack arrest fracture toughness results were similar for both the normalized, and normalized and stress relieved materials. 
Crack-Arrest Toughness near the Upper Shelf: International Consensus and Standards Research

Roland deWit and Richard J. Fields

Crack-arrest toughness near the upper shelf is a material property that is useful in assessing the safety of nuclear pressure vessels under thermal shock conditions. The objective of this program is to study high temperature dynamic fracture and crack arrest with the intention of establishing internationally accepted, standard methods for the performance of crack-arrest toughness tests near the upper shelf, standard analysis methods for the test data, and standard practices for the application of the results to actual structures such as nuclear pressure vessels.

Considerable effort and funding have been applied on a worldwide basis to acquire crack-arrest toughness data for a few pressure vessel steels. It appears at this time that existing, small specimen standard test methods are not adequate for the study of high temperature crack arrest, i.e., near the upper shelf. Most of the world's data on crack-arrest toughness near the upper shelf have been obtained from large specimens. Examples of such tests are: the French and American thermal shock experiments; the American pressurized thermal shock tests; the American, German, and Russian wide plate tests; the Japanese ESSO tests; and the British spinning cylinder tests. None of these large tests were performed in accordance with any standard because no standards exist for such tests.

Contact with selected major world efforts in dynamic fracture and crack arrest will be made under the auspices of the Versailles Treaty Advanced Materials and Standards (VAMAS) Organization. It is planned to form a Technical Working Area in crack arrest to coordinate efforts in Japan, Europe, and America. Success of the proposed effort would ultimately lead to improved understanding of crack-arrest toughness, how best to measure it, and how to use it.

\section{High Temperature Fracture of 316L Stainless Stee1}

Richard J. Fields

316L stainless steel is used in the construction of heat exchangers for advanced boiler systems. Recent failures in these systems suggested that there were gaps in the database of this alloy's fracture behavior at very high temperatures. Due to our previous published research on fracture mechanisms in 316 stainless steel, we were asked, by the Navy, to fill the technical knowledge gap for this material so that future boiler systems may avoid this problem.

The research consisted of a literature search and data compilation, a characterization of the material's condition in new and used boilers, high temperature mechanical tests and pulsed heating experiments, and metallurgical examination of the tested materials to deduce failure mechanisms. The literature survey and data compilation, and the pulsed heating experiments, have been completed. The mechanical testing and metallurgical examinations are in progress. Preliminary results suggest that weld cracks and stress corrodants are more likely responsible for the observed behavior than any new fracture mechanism. 


\section{Metal Matrix Composites}

Richard J. Fields and T. Robert Shives

Metal matrix composites (MMCs) represent the next generation of metallic materials and are on the verge of large scale commercialization. MMC refers to metals which have large fractions (10-50\%) of a strengthening phase (usually ceramic) intentionally added. The strengthening phase may be in the form of particles, whiskers, chopped fibers, or long fibers. Numerous U.S. firms are now in a position to supply MMCs by the ton. Manufacturing industries, such as automobile and aerospace, are extremely interested in these wear and creep resistant materials because they can improve their products' performance and efficiency. However, replacing traditional alloys with new advanced ones represents a gamble and there has been some reticence in the use of MMCs. The Metallurgy Division is carrying out research and other activities to facilitate the use of these materials in U. S. products.

One area of concern to U.S. industry, when substituting new materials, is long-term behavior, as this affects warranty considerations. We are studying the creep and fatigue behavior of MMCs, especially at elevated temperatures, to characterize the long-term behavior. Two important questions addressed by this research are: ( 1 ) How applicable are present standard test methods? and, (2) What are the microstructural bases for creep and fatigue resistance? An improved understanding will help the MMC industry produce better materials.

We are currently performing hot tensile, creep, and fatigue studies on a 6061 aluminum alloy strengthened with $20 \%$ by volume alumina microspheres, and toughness studies on a plasma sprayed 8090 alloy strengthened with silicon carbide particles. The $8090 / \mathrm{SiC}$ composite is being tested in a variety of heat-treated conditions to deduce the role of matrix properties on the behavior of the composite.

Industrial interactions in this project are numerous. We have met with a number of MMC producers and one is supplying materials for this research program. Recently one of our staff was appointed to an Aluminum Association Task Group on the development of aluminum matrix composites.

\section{Micromechanical Mechanisms of Solder Interface Failure}

Roland deWit

The objective of this task is to develop a better understanding of the coppertin interface in electronic solder connections. Two aspects were studied:

(1) The stresses and strains from the copper-tin intermetallics; and, (2) The diffusional growth of the copper-tin intermetallics.

An approximate calculation was made of the stresses and strains at a solder/intermetallic interface due to the growth of intermetallic particles of $\mathrm{Cu}_{6} \mathrm{Sn}_{5}$ from a copper substrate into tin. Initially, the growth of a spherical inclusion in a large body of tin was studied. Two extreme assumptions were made for the growth:

1) The inclusion grows by simply adding copper to it and forming an additional $\mathrm{Cu}_{6} \mathrm{Sn}_{5}$ layer from the reaction with the surrounding tin. In 
this case, the inclusion has a uniform dilatation of $e_{0}=0.432$.

2) The inclusion grows by replacing every 5 tin atoms around the inclusion with 6 copper atoms. In this case the uniform dilatation is

$e_{0}=-0.0597$.

In both cases the resulting strains are large enough to produce plastic flow around the inclusion. For a perfectly plastic material, the calculated plastic zone around the inclusion for case (1) is about eight times the inclusion radius and for case (2) about four times. In the plastic zone the difference between the circumferential and radial stresses equals the yield stress. Outside the plastic zone the stresses drop off with an inverse cube dependence. We conclude from this calculation that very large stresses are developed around the intermetallic phase. The next step would be to refine the calculation to approximate the observed interfaces more realistically.

Next we considered the problem of the diffusional growth of the copper-tin intermetallics at a flat copper-tin interface. William J. Boettinger formulated the moving boundary value problem. When the copper and tin are joined together, the intermetallics $\mathrm{Cu}_{3} \mathrm{Sn}$ and $\mathrm{Cu}_{6} \mathrm{Sn}_{5}$ begin to form and grow. The alloy concentration in each of the four phases is then determined by the diffusion equation. The solution is a linear expression in the error function, which depends on the position, the interdiffusion coefficient, and the time. Two constants of integration are determined by the concentrations at the phase boundaries, which are easily found from the copper-tin phase diagram. The motions of the three phase boundaries are determined by the solution of three nonlinear boundary value equations. A temperature of $200{ }^{\circ} \mathrm{C}$ was chosen as an example. To solve this problem, it is necessary to know the interdiffusion coefficient of copper and the two intermetallics, as well as the boundary concentrations from the phase diagram at this temperature. The result for the phase boundary positions is (meters, seconds):

$$
\begin{array}{ll}
\mathrm{x}=-6.2 \times 10^{-9} \mathrm{~J} t & \text { for the } \mathrm{Cu} / \mathrm{Cu}_{3} \mathrm{Sn} \text { boundary, } \\
\mathrm{x}=-3.2 \times 10^{-9} \mathrm{~J} t & \text { for the } \mathrm{Cu}_{3} \mathrm{Sn}_{\mathrm{Cu}} \mathrm{Sn}_{5} \text { boundary, } \\
\mathrm{x}=3.9 \times 10^{-9} \sqrt{ } \mathrm{t} & \text { for the } \mathrm{Cu}_{6} \mathrm{Sn}_{5} / \mathrm{Sn} \text { boundary. }
\end{array}
$$

Thus, the $\mathrm{Cu}_{6} \mathrm{Sn}_{5}$ grows about twice as thick as the $\mathrm{Cu}_{3} \mathrm{Sn}$ at this temperature. The copper concentration in each phase is practically constant at 1 in $\mathrm{Cu}$, 0.76 in $\mathrm{Cu}_{3} \mathrm{Sn}, 0.55$ in $\mathrm{Cu}_{6} \mathrm{Sn}_{5}$, and 0 in $\mathrm{Sn}$. This result is essentially a consequence of the low interdiffusion coefficient for copper in copper and the shape of the copper-tin phase diagram.

\section{Deformation and Consolidation of Metal Powder Compacts}

Richard J. Fields and Samuel R. Low, III

During the consolidation of metal powders, it is industrially desirable to control the deformation of the powder body in order to obtain near net shape in the final piece. To achieve such control, we have studied the mechanical behavior of partially densified metal compacts under uniaxial compression. The initial, elastic behavior is fully characterized by two elastic constants and the density. We have developed preliminary expressions for Young's modulus and Poisson's ratio as a function of density for pure titanium powder 
compacts. The plastic behavior of these compacts has been quantitatively separated into volume change, i.e., consolidation, and shape change as a function of applied hydrostatic and deviatoric stress states and density. The results of this research have been provided to industry and are being used as a benchmark that qualifies finite element simulations for modeling powder consolidation processing and improving the quality of powder metallurgy products.

\section{Modulus of Elasticity and Poisson's Ratio for Stainless Steels in Compression} T. Robert Shives and Richard J. Fields

At the request of Liberty Technologies of Conshohocken, PA, precision measurements were made by the Metalurgy Division of the modulus of elasticity and Poisson's ratio of specific samples of types 17-4 PH and 410 stainless steels in compression. These materials are intended for valve stems in nuclear reactor pressure vessel systems. The material for testing was supplied by the company.

The ends of the cylindrical specimens were machined flat and parallel. Axial loads not exceeding the elastic limit were applied and the axial and diametral displacements as functions of load were recorded. Total axial displacement during loading was less than $0.05 \mathrm{~mm}$ for the 17-4 $\mathrm{PH}$ material and less than $0.025 \mathrm{~mm}$ for the 410 material. Total diametral displacements for these materials did not exceed $0.0076 \mathrm{~mm}$ and $0.0038 \mathrm{~mm}$ respectively. Because of the exceedingly small strains involved, a number of determinations were made for each specimen tested. The average values obtained for both the modulus of elasticity and Poisson's ratio agree quite well with those reported in the literature for tensile stresses.

\section{Procedures for the Accreditation of Fastener Testing Laboratories}

Samuel R. Low, III, and T. Robert Shives

Each year, approximately 200 billion fasteners are sold and used in the United States. In recent years, millions of these fasteners have been found to be mismarked, substandard, counterfeit or nonconforming in some manner. Use of these nonconforming fasteners has threatened the health and safety of Americans, and also has harmed the manufacturers, importers, and distributors of safe and conforming fasteners.

To protect public safety, and to deter the introduction of nonconforming fasteners into commerce, the "Fastener Quality Act" (P.L. 101-592) was signed into law. The "Fastener Quality Act" requires that the Secretary of Commerce, acting through the Director of NIST, issue regulations establishing a program to accredit laboratories to inspect and test fasteners for certification as required by the Act. The Act also requires that NIST's National Voluntary Laboratory Accreditation Program (NVLAP) develop the procedures to be used to accredit laboratories.

NVLAP has asked the Metallurgy Division to provide technical support and assistance in drafting the procedures required for the accreditation of fastener testing laboratories in the areas of mechanical and performance testing. Hundreds of standards and specifications related to fastener testing 
were reviewed to identify the mechanical and performance tests required for fastener certification and acceptance. These documents include standards and specifications published by the federal government, military, international and national standards organizations, and American industry.

In developing the laboratory accreditation procedures, the Critical Elements that a laboratory must satisfy to adequately conduct each specific fastener test must be identified. The Critical Elements include items such as the required facilities, test equipment, calibrations, personnel and training procedures, and environmental conditions. Also required are Check Lists to be used by the assessors for evaluating a laboratory's ability to conduct specific tests. The Critical Elements and Check Lists for more than thirty fastener tests have been drafted, covering essentially all areas of mechanical and performance testing of metals. The procedures, however, are specific to the laboratory testing of metal fasteners. The areas of fastener laboratory testing include Mechanical Testing, Embrittlement, Hardness, Metallography, Corrosion, Measurement of Coating Thickness, Adhesion, Magnetic Permeability, and Non-destructive Inspection.

Assistance also was provided to NVLAP in designing a proficiency test program as an integral part of assessing the qualifications of testing laboratories seeking accreditation for certifying fasteners. As an aid to the proficiency test program, the development of standard reference fasteners having certain reproducible mechanical properties has been proposed.

Metallurgical Methods Used in the Intelligent Processing of Steel George E. Hicho and Lydon J. Swartzendruber

A program was initiated to determine if a correlation exists between the mechanical and physical properties, such as ultimate and yield strengths, reduction in area, elongation, ferrite grain size and microstructure and the magnetic properties of carbon steel sheet. Sheets of cold rolled and hot rolled steel of various thicknesses were obtained and the mechanical properties, microstructure, and ferrite grain size in the three orthogonal directions determined. Magnetic measurements also were conducted on these same samples. Results indicated that correlations exist between selected mechanical properties, in particular, the yield strength, and the magnetic properties. The research is continuing.

\section{Quantitative Metallography}

Richard J. Fields and Roland deWit

A new image analysis system was installed in the Metallurgy Division this year and work is underway to write codes to quantify the grain structure and inclusion contents of current and future steel products and alloys. The new system has numerous gray image modification and enhancement capabilities and can perform Boolean operations on the resulting binary images. These abilities are important in ensuring grain boundary connectivity and separating contiguous inclusions for correct sizing. Measurements are made on planar sections cut through three-dimensional objects and are, therefore, twodimensional measurements. A program is under development which will compute the three-dimensional microstructure from which the two-dimensional 
measurements were derived. We are investigating both neural networks and associative feature maps for this purpose. While the initial work is computationally intensive, it is hoped that the results will be readily implemented on the new image analysis system's computer or even on a standard personal computer for ease of dissemination.

\section{Hardness Standardization Project}

John H. Smith

The objective of this project is to develop a recognized National hardness standard in the United States that is based on the direct measurement of force, displacement and time, and that is traceable to NIST. This National hardness standard will be transferred to end users of hardness test machines by the use of "certified hardness calibration blocks" that are verified by NIST.

To develop a National hardness standard, it is necessary to construct a precision hardness test machine that can be verified to be substantially free from random and systematic errors in load, loading rate, and displacement. This will ensure the National hardness standard is accurate and that the hardness standard is maintained constant over time. This precision hardness machine will be used to verify "certified hardness calibration blocks" that can be used as reference standards to maintain a uniform hardness standard for all users.

During FY 91, the construction of a precision hardness machine was initiated. This machine is a "dead weight" type machine similar in basic design to the precision hardness machine at the Italian national measurement laboratory (IMGC) in Turin, which is used to maintain the standard European hardness scale. The actual construction of the U.S. machine is being carried out by the officine Galileo Company in Florence, Italy under the supervision of technical personnel from the IMGC laboratory.

For the N.I.S.T. precision hardness machine, design modification have been made to replace the electro-hydraulic control system used on the IMGC machine with a precision electronic control system to obtain more precise control of load, loading rate, and displacement of the hardness indentor during the hardness calibration tests. The design modifications to the basic dead weight hardness machine have been carried out as a collaborative effort between IMGC and N.I.S.T.

In addition to the construction of the precision hardness test machine at N.I.S.T., close collaboration has been established with all of the United States hardness machine and hardness calibration block manufacturers to facilitate the transfer of the National hardness standard to the hardness user community. Collaboration with other national laboratories in Italy, the United Kingdom, Australia, and Japan has been initiated to ensure that the U.S. National hardness standard is compatible with hardness standards worldwide.

A final report entitled "Intercomparison Study of Rockwell Hardness Test Blocks" (NISTIR 4531) on measurements of hardness blocks used in the United States was published in February 1991 and has been widely distributed within the hardness user community. 

The Metallurgical Processing Group studies the processing of metals and advanced materials to support U.S. industry and government through (1) research into the basic processing mechanisms and process modeling; (2) development of measurement techniques, systems, and data important for process monitoring and control; and (3) characterization of the phases and microstructures of the product materials as a function of processing conditions. Understanding of alloy kinetics and thermodynamics is pursued to provide guidelines that can be used by industry and alloy designers to tailor alloys and processing techniques for their particular applications.

The properties of materials depend on their compositions and microstructures. Therefore, if one wants to improve alloy properties, it is important to know how to control such material features as compositional homogeneity, alloy phases that form during solidification and other processing steps, interface reactions, and the resulting microstructures that can be produced. In research at NIST, strong emphasis is put on understanding how processing conditions influence compositional and microstructural distributions and on developing predictive models that will allow intelligent control.

As part of an "intelligent processing" program, ways to measure significant features of the material while the material is actually being produced are being developed. Examples are measurements of particle size distributions during powder atomization and densification of powder compacts during hot isostatic pressing. These measurements allow real-time feedback and control based on actual processing results. Industrial companies have shown considerable interest in this type of processing and have collaborated with NIST in this work, contributing both personnel and direct funding to aid NIST research in these areas.

Scientific understanding is particularly important in designing new high performance structural materials, such as high temperature intermetallics needed for aerospace applications and composites needed for a variety of commercial and defense applications. The Metallurgical Processing Group pursues work on these advanced materials as well as on materials having new electronic or magnetic applications, such as low-defect semiconductors, magnetic refrigeration materials, and high $\mathrm{T}_{c}$ superconductors. Much of this work is supported by other agencies, including DARPA, ONR, NASA, and the Navy's David Taylor Research Center.

In addition, new demands are being made on currently well-used materials, such as steel, aluminum alloys and solder. Development of new processing and alloying techniques for these materials show promise of significantly improving performance while still controlling costs. Examples include work with higher temperature aluminum alloys, powder atomized and nitrogenated steels, and surface-mount solder connections. NIST studies in these areas during the past year have been supported by several industrial companies and the Army's Harry Diamond Laboratories. 
- A new intermetallic heterophase alloy with superior mechanical properties has been obtained by producing a composite of two Ti-Al-Nb phases, one being an orthorhombic phase which is relatively ductile and the other an omega-type phase that is plastically unshearable. These phases are in equilibrium with each other up to $800^{\circ} \mathrm{C}$, thus providing stability in use.

- A unified approach, which uses crystallographic symmetry group/subgroup relations between phases, was developed to predict the complex transformation paths that change the high temperature BCC and ordered $B C C$ into the low temperature hexagonal, orthorhombic and omega-type phases in the $\mathrm{Ti}-\mathrm{Al}-\mathrm{Nb}$ system. Knowledge of these paths will permit an optimization of the heat treatments used for these alloys which have potential for reduced density and higher temperature performance in aerospace propulsion systems.

- A new method has been developed that predicts the growth patterns of solid particles forming from a melt during alloy solidification. This method treats the system as a whole using a continuous field variable to describe the state (liquid or solid) of the various regions of the system. Calculation of growth patterns and their Ostwald ripening is now possible for alloys and yields the composition segregation pattern left in final solid products. These segregation patterns are responsible for defects which limit the mechanical and corrosion properties of many alloys.

Microstructure/strength/toughness correlations in Al-Cu-Li based alloys as a plate product were examined in collaborative work with MartinMarietta Corp. It was found that best properties, which far exceed other aluminum alloys, appear to be associated with specimens containing 6 different strengthening precipitates.

The U.S. Industry/NIST atomization consortium has commenced a new three year study to demonstrate on-line control of particle size during gas atomization of Inconel 625, a nickel-based superalloy. This study will exploit intelligent processing results obtained in previous consortium work. Members of the consortium include Crucible Materials Corp., General Electric Co., Martin Marietta Energy Systems, Inc., Pratt \& Whitney Aircraft, and the Office of Industrial Processes of the U.S. Department of Energy. NIST participants include the Metallurgy Division, the Chemical Process Metrology Division, the Precision Engineering Division, and the Factory Automation Systems Division.

The melting capacity of the Metallurgy Division's SiGMA system (Supersonic inert Gas Metal Atomization system), used to produce spherical metal powder $50 \%$ by weight $\leq 20 \mu \mathrm{m}$ in diameter, was increased to $23 \mathrm{~kg}$ ( $50 \mathrm{lb}$ steel measure).

The microstructure of atomized powders of $\mathrm{Cu}_{6} \mathrm{Sn}_{5}$ was analyzed before and after consolidation by hot isostatic pressing (HIP), and processes 
leading to inhomogeneity of the densified product were identified. These studies indicated modifications of the HIP process which yielded a more uniform product. This result may be broadly applicable to consolidation of other intermetallic compounds.

A study of the $\mathrm{Al}-\mathrm{Cu}-\mathrm{Fe}$ phase diagram was completed, establishing the equilibria that occur with the icosahedral phase at 700 and $800^{\circ} \mathrm{C}$. A new metastable phase was found at $550^{\circ} \mathrm{C}$.

Grain boundary grooving experiments have been performed on tin bicrystals in contact with eutectic $\mathrm{Sn}-\mathrm{Pb}$ liquid to measure the product of the liquid diffusion coefficient and the solid-liquid surface tension. This measurement is important to allow interpretation of coarsening results in the $\mathrm{S} n-\mathrm{Pb}$ system.

A new phenomenon was found in tilt boundaries of superconducting $\mathrm{YBa}_{2} \mathrm{Cu}_{3} \mathrm{O}_{6+x}$ which links transformation strains across grain boundaries. This effect may have importance in connection with the high critical currents found in some of the promising highly textured superconductors.

\section{Powder Processing and Process Control}

S. D. Ridder, F. S. Biancaniello, R. D. Jiggetts, R. J. Schaefer

Powder processing activities are focused on application of intelligent processing techniques using real-time measurements to control the production and consolidation of metal powders. The Metallurgical Processing Group's gas atomization program completed a three year consortia-managed effort to apply intelligent processing to particle size control and began the second phase of this effort (also scheduled for three years). The second phase activity continues the cooperative effort with three other NIST divisions and several US companies. Intelligent processing techniques were also applied to HIP consolidation processes in collaboration with DARPA and the BDM Corporation.

Powder Atomization - The capacity of the Metallurgy Division's Supersonic inert Gas Metal Atomization system (SiGMA) has been increased to $23 \mathrm{~kg}$ (50 pounds steel measure). The SiGMA atomization capabilities which previously focused on $304 \mathrm{SS}$, were extended to include $\mathrm{Ni}$ based superalloys with a current emphasis on Inconel 625. Parallel studies on the advantages of applying rapid solidification to gas atomized powder included studies of nitrogenated steels and the intermetallics found in electrical solder joints. Also other difficult-to-process materials, applicable to magnetic refrigeration, were produced in powder form by atomization.

Automation .. Real-Time Powder Particle Size Measurement and Control - The NIST/Industrial consortium on powder processing began a three year multidisciplinary study on automation utilizing the supersonic inert gas metal atomizer (SIGMA). NIST research groups include the Intelligent Control Systems Group of the Factory Automation Systems Division, the Surface and Particle Metrology Group of the Precision Engineering Division, the Fluid Flow Group of the Chemical Process Metrology Division and the Metallurgical Processing Group of the Metallurgy Division. The NIST scientists collaborate in this work with consortium scientists from the Crucible Materials Corp., 
General Electric Co., Martin Marietta Energy Systems, Inc., Pratt \& Whitney Aircraft, and the Office of Industrial Processes of the U.S. Department of Energy.

This second phase activity will incorporate the in-situ particle size measurement sensor and expert-system-driven process controller developed in the first phase of the consortium work in a study focused on the atomization of a nickel-based super alloy (Inconel 625). During this year, work has continued on the development of advanced computer systems and sensors for real-time measurement and control of powder size. The particle measurement sensor combines Fraunhofer diffraction optics and novel pattern recognition software to achieve the necessary data transfer rate of $2 \mathrm{hz}$. The Fraunhofer diffraction particle size sensor used in the first phase activity has recently been modified and now uses a search-optimization pattern recognition software to resolve scattered laser intensity data into a distribution of 14 different sizes between 5 and $150 \mu \mathrm{m}$. This novel approach to particle size analysis, developed by NIST researchers, improves the accuracy of Fraunhofer diffraction analysis by several orders of magnitude compared to current iterative techniques and can resolve difficult distributions such as monodispersed and "square wave" shaped multi-modal distributions. The controller runs an "expert system" shell processing sensor data and sending appropriate actuator signals to maintain or change the particle size distribution as directed by the heuristic data base and response functions "learned" during previous programming sessions.

Fluid flow studies have also continued during the second phase work. High speed movies of metal atomization are now illuminated with a 12 watt Cu-vapor laser synchronized with the 10,000 frames per second (fps) camera. This improves the temporal resolution from $10 \mu \mathrm{s}$ to $20 \mathrm{~ns}$ and allows movies to be made when atomizing low temperature, non-incandescent alloys. Mathematical modeling of supersonic gas flow has continued with Navier-Stokes and Euler solutions now running in the Cray Y-MP. A compiled version of this code (suitable for running on PC computers) has been developed for use by consortium members and is currently in use by the Crucible Materials Corp. This code is now being modified to incorporate automatic grid optimization to improve flow property resolution.

Hip Consolidation of Metal Powders - The consolidation of several types of material by hot isostatic pressing (HIP) has been studied with the objective of obtaining better control of product microstructure, density, and shape. The HIP process is especially useful for consolidation of intermetallic compounds and composite materials to near-net shape, because of the difficulty of forming these materials by conventional processes. The Metallurgy Division has completed work on a project sponsored by DARPA, in which it worked with BDM International, the University of Cambridge, and an Industrial Consortium to apply techniques of intelligent processing of materials (IPM) to HIP consolidation of powders of titanium and titanium aluminide. It also has completed a study of the densification of powders of intermetallic phases which form in solder joints, sponsored by the U.S. Army Harry Diamond Laboratories. 
HIP densification of titanium aluminide powders was found to be enhanced during the part of the HIP cycle in which the powders transform from the initial metastable $\alpha_{2}$ crystal structure to an equilibrium mixture of $\gamma$ and $\alpha_{2}$ phases. After investigating several possible sources of this effect, it was concluded that it was due to transformation plasticicity, which is an increased plastic strain experienced by a material undergoing a phase transformation when subjected to stress. HIP densification of commercial purity titanium was carried out part way to completion to provide samples for subsequent testing in non-hydrostatic stress states. These experiments investigated the plastic yield surface for particulate materials and compared it to a variety of theoretical models which have been proposed as the basis of finite element modeling of shape changes in HIP samples.

A detailed study of the phases present in atomized $\mathrm{Cu}_{6} \mathrm{Sn}_{5}$ powder and their distribution in HIP consolidated samples revealed that during the HIP cycle free tin present in the initial powder can melt and flow into pores present in some of the larger powder particles. This segregation process delays transformation of the HIP sample to its equilibrium single-phase $\mathrm{Cu}_{6} \mathrm{Sn}_{5}$ condition. More homogeneous samples were produced by modifications of the sample preparation which reduced the probability of the HIP-induced segregation process.

\section{Interface and Solidification studies}

J. R. Manning, W. J. Boettinger, S. R. Coriell, C. A. Handwerker and S. C. Hardy

Almost all metals that find use as manufactured parts are melted at some time during their processing. Thus, reactions occurring at solid-liquid interfaces during solidification play a major role in determining composition distributions and microstructures in the finished part. Further reactions occur at solid-solid interfaces and grain boundaries in the solidified metals. These interface reactions play key roles in determining final properties.

New Calculational Method for Pattern Formation during Alloy Solidification - A new method has been developed that predicts the growth patterns of solid particles forming from a melt during alloy solidification. This method treats the system as a whole using a continuous field variable to describe the state (liquid or solid) of the various regions of the system. The interfacial region is thus necessarily diffuse. This method, while relatively simple from a computational point of view involving only the solution to a system of parabolic partial differential equations, includes a wide variety of physical phenomena such as nonequilibrium kinetics and capillarity. Classical methods used to calculate these growth morphologies involve numerical tracking of the free boundary that separates the liquid from the solid and individual treatment of each phase that result in very difficult and untidy numerical algorithms. This new method was previously developed for pure materials. Through NIST work involving collaboration between the Metallurgy and the Applied and Computational Mathematics Divisions, this method has now been applied to the more useful case of binary alloys. Calculation of growth patterns and their Ostwald ripening is now possible for alloys and yields the 
composition segregation pattern left in final solid products. These segregation patterns are responsible for defects which limit the mechanical and corrosion properties of many alloys.

Interface Migration Produced by Diffusion - Solute diffusion will generate strains in the diffusion zone of a solid if the lattice parameter changes with composition; as a result of these strains, the interfaces from which the solute is supplied may be destabilized. This destabilization can take two forms: preexisting interfaces may migrate, often with a characteristic "sinusoidal" morphology, or new grains may form and migrate into the unalloyed solid. Typical examples of diffusion-induced migration with a sinusoidal morphology are diffusion-induced grain boundary migration (DIGM), liquid film migration (LFM), in which the liquid film between two solid grains migrates, and the interface instability of a single solid-liquid interface (ISL). The nucleation of new grains resulting from diffusion is known in the literature as diffusion-induced recrystallization (DIR). In the current work it has been found that the choice concerning which of these two morphologies the system exhibits depends on the initial microstructure, the temperature and the magnitude of the strain produced by diffusion. Diffusion-induced recrystalization (DIR) dominates at higher temperatures and strains.

Recently developed geometric models of interface motion have been used to predict the range of growth shapes possible for growing DIR grains for cubic systems as a function of the elastically anisotropic factors in the coherency strain energy. The mathematical technique known as the method of characteristics has allowed us to make precise statements about limiting growth morphologies of DIR grains when the interface velocity is a known function of interface normal. The limiting growth shapes for DIR can be characterized as a function only of the three independent cubic elastic coefficients. From these calculations, the extent and position of any edges and corners which develop in the limiting growth shape have been predicted. The experimentally observed shapes of DIR grains in the $\mathrm{Fe}-\mathrm{Zn}$ and $\mathrm{Mo}-\mathrm{Ni}$ systems agree with the predicted shapes. This work also has established mathematical and computational techniques for subsequent application to the other nine anisotropic sets of elastic coefficients and to the other diffusion-induced processes of DIGM, LFM, and ISL.

Solidification Interfaces - Radiative heating of silicon is an important processing technique for silicon wafers. Two-phase mixtures of solid and liquid silicon form during the laser processing of thin silicon films on substrates such as fused silica. The morphology of the solid-liquid interfaces may be either planar, cellular, or dendritic. The higher reflectivity of liquid silicon compared to solid silicon allows the formation of superheated solid adjacent to supercooled liquid. In collaboration with $L$. N. Brush of the University of Washington and G. B. McFadden of the Computing and Applied Mathematics Laboratory, the linear stability analysis of Jackson and Kurtze has been extended to finite amplitudes by developing a boundary integral representation of the thermal field. Steady-state solutions for highly non-linear solid-liquid interfaces are computed, and the stability of these nonlinear solutions are determined numerically. The change in interface morphology as the laser power increases has been investigated. 
Grain Boundary Grooving Experiments - The solid-liquid surface tension and liquid diffusion coefficients are of importance for a number of metallurgical phenomena including the coarsening of solid-liquid mixtures and the dendritic growth of metallic alloys. The product of the solid-liquid surface tension $\tau$ and the liquid diffusion coefficient $D_{\underline{L}}$ determines the rate of grain boundary grooving in an isothermal system. Experimental measurements of isothermal grain boundary grooving by volume diffusion have been carried out for tin bicrystals in the lead-tin system near the eutectic temperature (see figures 1 and 2). The dimensions of the groove increase with the one third power of the time, and the product $\tau D_{\mathrm{L}}$ can be determined by measurement of the rate constant for grooving. Previous theoretical analysis of grain boundary grooving assumed that the solid-liquid interface was nearly planar, which is difficult to achieve experimentally. In collaboration with G. B. McFadden of the Computing and Applied Mathematics Laboratory, the small-slope theory of Mullins has been extended to large slopes by using a boundary-integral formulation of the associated free boundary problem. Excellent agreement is obtained between computed and experimental grain boundary groove shapes, allowing an accurate determination of the product $\tau D_{\underline{L}}$, which agrees to within $5 \%$ with previous independent measurements.

\section{Physical Metallurgy and Processing of Intermetallics and Advanced Aluminum} Alloys

W. J. Boettinger, L. A. Bendersky, F. W. Gayle, U. R. Kattner*, B. P. Burton, F. S. Biancaniello, M. E. Williams and J. W. Cahn**

* Guest Scientist - University of Wisconsin - Madison

** Laboratory Scientist

Considerable excitement has been generated in the metallurgical community by the potential for significant improvements in aerospace materials using intermetallic and advanced aluminum - lithium alloys. Research in this task is concerned with improvements in the metallurgical understanding of these materials. This information permits a rational approach to the control of microstructure and properties through processing. Included in this task are phase equilibria and phase transformation studies on a broad range of $\mathrm{Ti}$ and $\mathrm{Ni}$ aluminides and $\mathrm{Al}-\mathrm{Ii}-\mathrm{Cu}$ alloys.

Phase Transformations in Ti-Al-Nb Intermetallic Alloys - Based on previous research under this task involving the ordered omega phase, a patent application has been made for a series of $\mathrm{Ti}-\mathrm{Al}-\mathrm{Nb}$ alloys in the following composition range: 48-52 at\% $\mathrm{Ti}, 28-32$ at\% $\mathrm{Al}, 16-20$ at\% $\mathrm{Nb}$. The alloys are designed to have low density, room temperature ductility and high-temperature strength and stability for possible aerospace applications. The high strength and microstructural stability is achieved when these alloys are induced by proper heat treatment to form a natural (in thermodynamic equilibrium) composite of relatively ductile orthorhombic $\mathrm{Ti}_{2} \mathrm{AlNb}$ and plastically unshearable omega-type $\mathrm{B}_{2}$ intermetallic phases. In the series of alloys covered by this invention, the omega-type phase is used for such purposes for the first time.

Continued research on phase transformations in $\left(\mathrm{Ti}(1-x) \mathrm{Nb}_{x}\right)_{3} \mathrm{Al} \quad(0<\mathrm{x}<0.34)$ alloys has been conducted using TEM and crystallographic space group analysis. These compositions include many which are currently being used for the 
fabrication of titanium aluminide intermetallic matrix composites. Upon cooling from the $B / B 2$ phase field these alloys exhibit a complex

transformation path into hexagonal and orthorhombic phases which depends on composition and cooling rate. As with most $\mathrm{Ti}$ alloys the properties are very sensitive to the heat treatment schedule employed. The transformation path establishes a hierarchy of microstructural scales which allows one to trace the transformation sequence. A method that considers reductions in symmetry according to the crystallographic group/subgroup relations between the involved phases was applied to this system for the first time. The group/subgroup relation together with considerations of minimum elastic energy enables one to predict the transformation path and the main microstructural features of the domain structures formed by chemical and displacive ordering.

Ti-Al-Mb Phase Diagram Modeling - In order to assimilate the above information and the phase diagram data of four other research groups, a thermodynamic calculation of the Ti-Al-Nb system is being performed. Such calculation begins from the three binary diagrams. Recently published data required a refinement of the analytical descriptions of the binary $\mathrm{Nb}-\mathrm{Al}$ and $\mathrm{Ti}-\mathrm{Al}$ systems. Results from these binary evaluations are shown in Figure 3.

For the Ti-Al system, the equilibrium phases are: the disordered solution phases, liquid, $\alpha-\mathrm{Ti}$ (hcp), B-Ti (bcc) and Al (fcc); and the ordered intermetallic compounds, $\mathrm{Ti}_{3} \mathrm{Al}\left(\alpha_{2}\right)$, which crystallizes in an ordered hcp structure, TiAl $(\gamma)$ and $\mathrm{TiAl}_{3}$, both of which crystallize in ordered fcc structures. The accurate phase relationships in the range between TiAl and $\mathrm{TiAl}_{3}$ are still subject to discussions in the literature, due to the occurrence of long period fcc-based superstructures. In the present calculation only $\mathrm{TiAl}_{2}$ and $\mathrm{Ti}_{2} \mathrm{Al}_{5}$ are considered. Three different equations were used to describe the concentration dependence of the Gibbs energies of the different types of phases occurring in the Ti-Al system. The disordered solution phases were described by regular solution type models. The ordered intermetallic compounds, $\mathrm{Ti}_{3} \mathrm{Al}$, TiAl and $\mathrm{TiAl}_{3}$, reveal more or less wide ranges of homogeneity and are considered to consist of two sublattices with Ti and $A 1$ occupying each of these sublattices. The $T i A l_{2}$ and $T i_{2} \mathrm{Al}_{5}$ phases were assumed to be stoichiometric. The final number of parameters necessary was determined during the least squares optimization with a selected data set.

The equilibrium phases in the $\mathrm{Nb}-\mathrm{Al}$ system are: the disordered solution phases, liquid, bcc-( $\mathrm{Nb})$ and $f c c-(\mathrm{Al})$; and the ordered intermetallic compounds, $\mathrm{Nb}_{3} \mathrm{Al}, \mathrm{Nb}_{2} \mathrm{Al}$ and $\mathrm{NbAl}_{3}$. The disordered solution phases were described by regular solution type models. The ordered intermetallic compounds, $\mathrm{Nb}_{3} \mathrm{Al}, \mathrm{Nb}_{2} \mathrm{Al}$ and $\mathrm{NbAl}_{3}$, reveal more or less wide ranges of homogeneity and are considered to consist of different sublattices with $\mathrm{Nb}$ and Al occurring on each of these sublattices. $\mathrm{Nb}_{3} \mathrm{Al}$ and $\mathrm{NbAl}_{3}$ were each described with two sublattices, allowing substitutional solution to occur on each of these sublattices. $\mathrm{Nb}_{2} \mathrm{Al}$ was described with three sublattices of a compound having the stoichiometry $\mathrm{Nb}_{16} \mathrm{Al}_{10} \mathrm{Nb}_{4}$ at perfect ordering and substitutional solution occurring only on the $\mathrm{Nb}_{16}$ and $\mathrm{Al}_{10}$ sublattices. For the least squares optimization of the parameters a selected data set from the literature was used. The course of the least squares optimization revealed 
that two sets of thermodynamic values in the selected data set were inconsistent with the phase diagram data and were, therefore, omitted for the final calculation.

Ordering and Phase Separation in $\mathrm{Ni}_{3} \mathrm{Al}_{-\mathrm{Ni}} \mathrm{V}$ alloys - The pseudo-binary $\mathrm{Ni}_{3} \mathrm{Al}$ $\mathrm{Ni}_{3} \mathrm{~V}$ phase diagram has been selected to study the kinetics of the phase transformation from the high temperature (HT) disordered fcc phase to the low temperature (IT) ordered $\mathrm{Ll}_{2}\left(\mathrm{Ni}_{3} \mathrm{Al}\right)$ and $\mathrm{DO}_{22}\left(\mathrm{Ni}_{3} \mathrm{~V}\right)$ phases. Both $\mathrm{LT}$ phases are subgroups of the HT phase but not of each other. Such a situation poses an interesting problem of phase transformation mechanism involving congruent ordering and phase separation. Crystallographic details of the transformation in three alloys, $\mathrm{Ni}_{75} \mathrm{Al}_{15} \mathrm{~V}_{10}, \mathrm{Ni}_{75} \mathrm{Al}_{5} \mathrm{~V}_{20} \mathrm{Ni}_{80} \mathrm{Al}_{15} \mathrm{~V}_{5}$, both cast and rapidly solidified, are being investigated by means of TEM. The alloys may have potential as a superalloy-class material.

First Principles Theoretical Studies of the Ni-Al-Ti System - A first principles approach, combining band theory calculations of cohesive energies and the cluster variation method of phase diagram calculations has been applied for the first time to a ternary alloy system. In collaboration with $A$. Pasturel (CNRS Grenoble), the following have been calculated: 1) the NiAl-NiTi pseudobinary phase diagram; 2) Ternary bcc based ground-states for the system $\mathrm{Ni-A1-Ti;} \mathrm{and} \mathrm{3)} \mathrm{Critical} \mathrm{temperatures} \mathrm{for} \mathrm{the} \mathrm{L} 2_{1} \rightarrow \mathrm{B} 2$ and $\mathrm{B} 2 \rightarrow \mathrm{bcc}$ transitions in $\mathrm{Ni}_{2} \mathrm{AlTi}$. Partial results of the NiAl-NiTi calculations were published (MRS Symp. Proc.213), and a more complete paper has been accepted for publication in Phys. Rev. B. Preliminary results of the full ternary calculation were reported at the European Workshop on Ordering and Disordering in Alloys (Grenoble 7/91).

Aluminum-base Alloys - A new class of ultra-high strength, weldable aluminum-copper-lithium alloys was recently developed by Martin-Marietta Laboratories. These alloys, originally targeted for the Advanced Launch System, have twice the yield strength of the baseline alloy (2219) used in the Space Shuttle External Tank and hold promise for a quantum jump in structural alloys for aerospace and other applications. Research at NIST has been directed at determining the microstructural basis for the high strength and toughness of these alloys. We have found that the system contains a variety of strengthening precipitate phases, ranging from a metastable equilibrium structure of $\mathrm{Al}, \mathrm{GP}$ zones and $\delta^{\prime}\left(\mathrm{Al}_{3} \mathrm{Li}\right)$ in the naturally-aged condition to a combination of three or four strengthening phases in the peak strength condition: $\mathrm{T}_{1}\left(\mathrm{Al}_{2} \mathrm{CuLi}\right), \mathrm{S}^{\prime}\left(\mathrm{Al}_{2} \mathrm{CuMg}\right), \theta^{\prime}\left(\mathrm{Al}_{2} \mathrm{Cu}\right)$, and a possible new phase $(\nu)$. Emphasis this year has been on commercially produced plate products. In the slightly under-aged condition (less than peak strength) the product has strength and toughness which far exceed that of other aluminum alloys. These properties are associated with a remarkable combination of at least six strengthening phases, including $\mathrm{T}_{1}, \mathrm{GP}$ zones, $\theta^{\prime}, \mathrm{S}^{\prime}, \Omega\left(\mathrm{Al} \mathrm{I}_{2} \mathrm{Cu}\right), \nu$, and a phase that, from preliminary examination, appears to be $\mathrm{T}_{B}\left(\mathrm{Al}_{15} \mathrm{Cu}_{8} \mathrm{Li} \mathrm{i}_{2}\right)$.

Aluminum-Copper-Iron Phase Equilibria. The Al-Cu-Fe ternary system contains the most perfectly ordered quasicrystal to date, which is an equilibrium phase of approximate composition $\mathrm{Al}_{65} \mathrm{Cu}_{24} \mathrm{Fe}_{11}$. The phase is based on a $6-\mathrm{D}$ facecentered cubic lattice, in contrast to other icosahedral phases which are simple cubic (6-D). The ternary phase diagram surrounding the icosahedral 
phase had not been investigated in detail previously. We have completed an experimental investigation of this region (50-75at.\% Al, $0-25 \% \mathrm{Fe}$ ).

Isothermal sections have been determined at $680^{\circ} \mathrm{C}, 700^{\circ} \mathrm{C}, 720^{\circ} \mathrm{C}$ and $800^{\circ} \mathrm{C}$, and all phases in equilibrium with the icosahedral phase have been identified. The range of solubility of the icosahedral phase has been determined at these temperatures, which should facilitate the study of non-stoichiometric defect structures and location of the atoms in quasicrystalline phases. Four-phase invariant reactions have been located and a liquidus surface proposed. A new metastable phase with a large unit cell based on the B2 (CsC1) structure has been found at $550^{\circ} \mathrm{C}$.

Special Materials -- Composites, Solder Intermetallics and High $\mathbf{T}_{c}$ Superconductors

C. A. Handwerker, W. J. Boettinger, F. W. Gayle, B. P. Burton and

J. R. Manning

Processing-microstructure-property relations are being investigated in a variety of materials, including steel, superalloys, high temperature intermetallics, and aluminum alloys, as discussed in previous sections. In addition, work on composites, solder joint materials and high $\mathrm{T}_{\mathrm{c}}$ superconductors is being done. Accomplishments in these work areas are described here.

Interdiffusion in the Ni-Graphite Composite System - The physical processes responsible for the degradation of nickel-graphite composites during high temperature annealing were investigated for graphite-fiber composites formed by electrodeposition in a research program with Nea Wheeler of American Cyanamid and David Lashmore and Alexander Shapiro of the Metallurgy Division. Turbostratic graphite fibers were observed to undergo dramatic changes in morphology during annealing in a nickel matrix at temperatures between $600^{\circ}$ and $1100^{\circ} \mathrm{C}$. As had been observed previously, graphite has a high solubility in $\mathrm{Ni}$ and the fibers dissolve readily by carbon diffusion into the nickel matrix. In this study, the morphology of the fiber-matrix interface and the dissolution kinetics were strongly dependent on annealing temperature. At $600^{\circ} \mathrm{C}$ the fiber-matrix interface was irregular, but maintained an approximately circular cross section. At $800^{\circ} \mathrm{C}$ the fiber-matrix interface showed pronounced "notching" with the size of the scallops/notches being approximately equal to the size of the nickel grains surrounding the fiber. At temperatures on the order of $1100^{\circ} \mathrm{C}$, the fibers recrystallized and isolated nickel grains were observed within fibers. Grain growth in nickel matrix was observed; the original structure was heterogeneous, and the final structure was affected by the starting heterogeneity and by the presence of the fibers.

A kinetic analysis of diffusion over this temperature range was performed using diffusion into a finite $\mathrm{Ni}$ matrix from a cylindrical source of carbon. At $600^{\circ} \mathrm{C}$ the dissolution rate of cylindrical graphite fibers in a nickel matrix was of the same order of magnitude as that predicted from diffusion theory, but diffusion at $\mathrm{T}>800^{\circ} \mathrm{C}$ occurred several orders of magnitude more slowly than predicted. This lower-than-predicted dissolution rate and the notched matrix-fiber interface are a result of separation of fibers from the matrix along some but not all areas of the fiber-matrix interface during heating to annealing temperature. 
The Wetting of the Intermetallics $\mathrm{Cu}_{6} \mathrm{Sn}_{5}$ and $\mathrm{Cu}_{3} \mathrm{Sn}$ by $\mathrm{Pb}-\mathrm{Sn}$ - The intermetallic compounds $\epsilon-\mathrm{Cu}_{3} \mathrm{Sn}$ and $\eta-\mathrm{Cu}_{6} \mathrm{Sn}_{5}$ are commonly found in solder joints as thin reaction layers separating $\mathrm{Cu}$ base metal and solder. For protection from oxidation for subsequent soldering operations, copper connectors are often pretinned by either hot dipping in $\mathrm{Pb}-\mathrm{Sn}$ or electroplating with $\mathrm{Pb}-\mathrm{Sn}$. During storage for extended periods these intermetallics can grow through the protective layer and greatly limit subsequent solderability. Thus the wetting behavior of the intermetallic compounds themselves is of great importance.

Area of spread tests have been performed at $235^{\circ} \mathrm{C}$ for $\mathrm{Sn}-40 \mathrm{wt} \% \mathrm{~Pb}$ solder on bulk samples of the intermetallics which are usually found in solder joints as well as on pure $\mathrm{Cu}$. Single phase $\mathrm{Cu}_{6} \mathrm{Sn}_{5}$ and $\mathrm{Cu}_{3} \mathrm{Sn}$ samples were prepared by HIP consolidation of atomized powder. The wetting behavior is most strongly dependent on the activity of the flux used. For a given flux, the measured areas and contact angles show that the wetting of $\mathrm{Cu}_{6} \mathrm{Sn}_{5}$ and $\mathrm{Cu}_{3} \mathrm{Sn}$ is much poorer than for $\mathrm{Cu}$. Significant variability in the wetting behavior is observed for $\mathrm{Cu}_{6} \mathrm{Sn}_{5}$ using a nonactivated rosin flux with contact angles ranging between $35^{\circ}$ and $180^{\circ}$. Preliminary wetting balance tests revealed a strong degradation of wetting behavior of the intermetallics with storage time.

Experimental Phase Equilibria/Crystal Chemistry of Oxide Superconductors and Related Systems - Oxide phases in the system Ca-Bi-Sr-Cu-0, have been synthesized and characterized and their equilibrium phase relations have been determined. This work clarified the multicomponent phase equilibrium of the two- and three-component subsystems of the five component system in which high $T_{c}$ oxide superconductors have been found. Knowledge of the phase equilibrium. has facilitated the processing of the high $T_{c}$ phases. This work has been done in collaboration with C.C. Torardi (E.I. duPont) and J.B. Parise (SUNY-Stony Brook and E.I. duPont).

High $T_{c}$ Superconductors - Grain Boundary Characterization - Grain boundaries in high temperature superconductors are a major "weak link" to electrical transport in polycrystalline specimens. The present study used bi-crystals to analyze a special class of grain boundary commonly found in the promising, highly crystallographically-textured "melt-processed" superconductors. Fiftytwo facetted grain boundary segments ([001] tilt boundaries) in clusters of bulk-scale $\mathrm{YBa}_{2} \mathrm{Cu}_{3} \mathrm{O}_{7-x}$ crystals having coincident c-axes were characterized by optical microscopy techniques. Grain boundary orientations were widely distributed. All grain boundaries, except those far from the symmetric condition and those with (110) facets, exhibited well-developed matching of the twin domains across the boundary. Our model of transformation strain indicates that this newly-reported phenomenon of twin pattern matching occurs due to a local coordination of the tetragonal $\rightarrow$ orthorhombic transformation strain across grain boundaries. This may be beneficial with regard to electrical transport in highly-textured polycrystalline material due to decreased crack development, improved dynamic electron coupling near grain boundaries, and the microtexture found near these grain boundaries. 
High $\mathrm{T}_{c}$-Superconductors - Detwinned Single Crystals - The development of a thermomechanical process for detwinning $\mathrm{YBa}_{2} \mathrm{Cu}_{3} \mathrm{O}_{6+x}$ single crystals in FY89 (with D. Kaiser, Ceramics Div.) has presented opportunities for numerous characterization studies of single crystals without the complication of twinning defects. Furthermore, this year comparative investigations on twinned and detwinned crystals were conducted to elucidate the effect of twin boundaries on properties. Magnetic measurements (with L. Swartzendruber and L. Bennett, Magnetic Materials Group, and A. Roytburd, U. Maryland) on a single crystal of $\mathrm{YBa}_{2} \mathrm{Cu}_{3} \mathrm{O}_{6+x}$ in the twinned and detwinned states have demonstrated that flux pinning by twin boundaries is strongly dependent on temperature and the magnitude and orientation of the applied field with respect to the crystallographic orientation. For applied magnetic fields perpendicular to the c-axis, no effect of twin boundaries on flux pinning was found at any temperature. In contrast, for fields up to $31.8 \mathrm{kA} / \mathrm{m}$ aligned parallel to the c-axis, the hysteresis loops at $77 \mathrm{~K}$ clearly showed increased pinning due to twin boundaries. At $40 \mathrm{~K}$ and low fields $(\mathrm{H}<39.8 \mathrm{kA} / \mathrm{m})$ the flux pinning was nearly the same for the twinned and detwinned states, whereas at higher fields (up to $3.2 \mathrm{MA} / \mathrm{m}$ ), pinning was higher in the twinned state. At this temperature, a tendency toward flux depinning occurred in the detwinned state at about $0.8 \mathrm{MA} / \mathrm{m}$, and both the twinned and detwinned states exhibited a more rigid flux structure at $4 \mathrm{MA} / \mathrm{m}$ than at $0.8 \mathrm{MA} / \mathrm{m}$. 


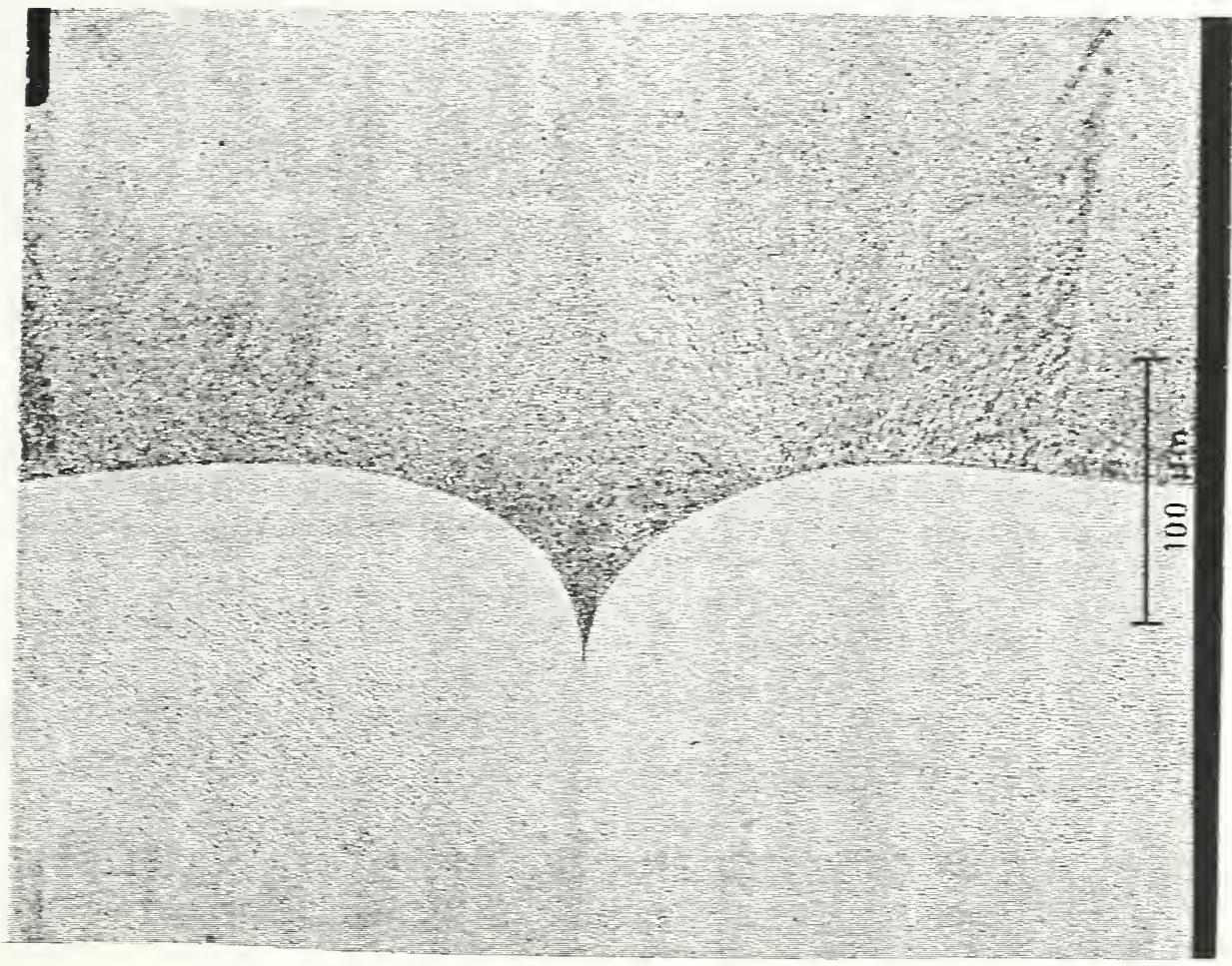

Figure 1. Experimental photograph of grain boundary groove that formed in a tin bicrystal in contact with lead-tin liquid. 
$\theta=84$

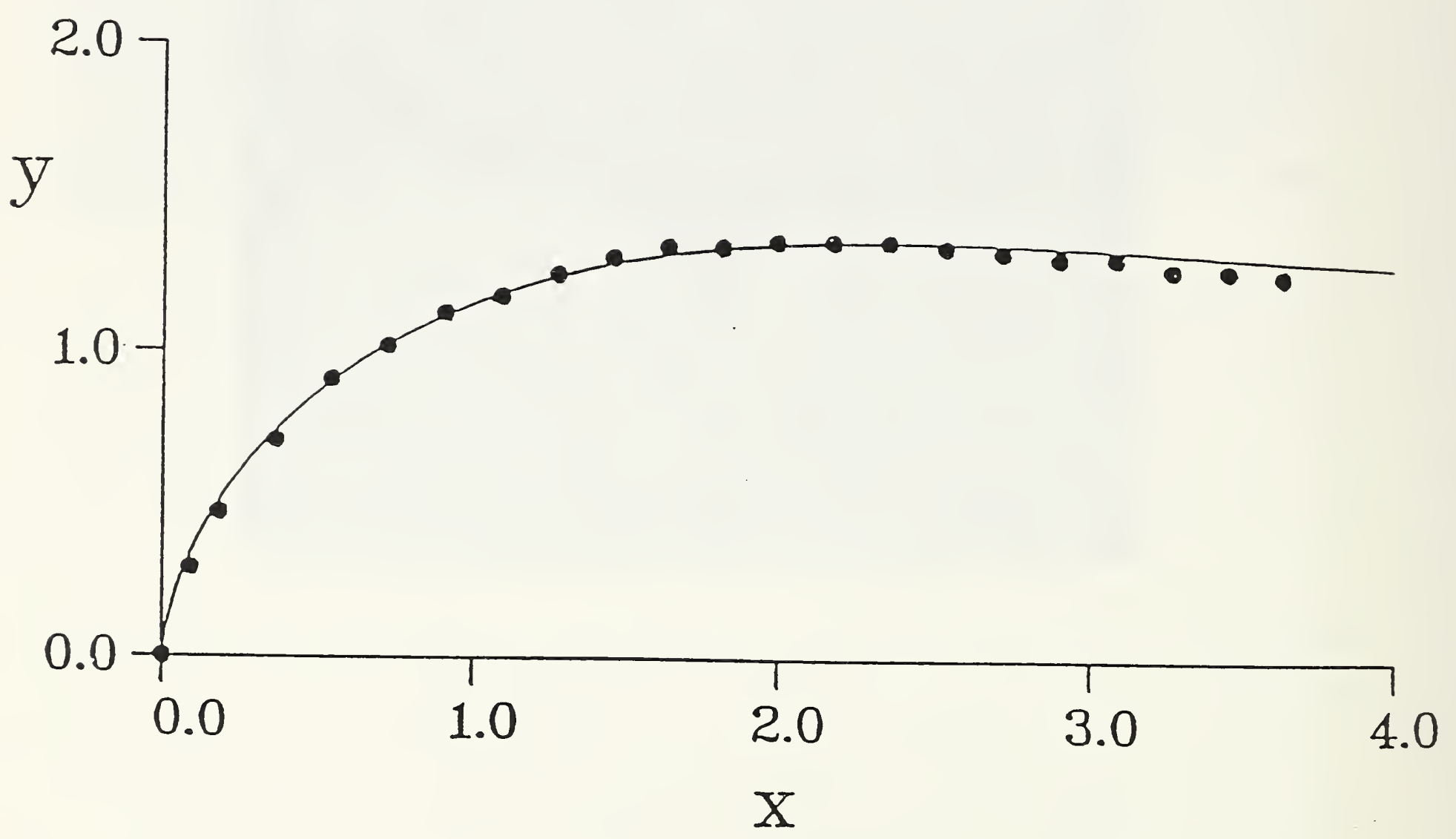

Figure 2. Comparison of theoretical shape of grain boundary groove, shown by solid curve, with experimentally measured position of solid-liquid interface, shown by black circles, for solid tin in contact with lead-tin liquid. 

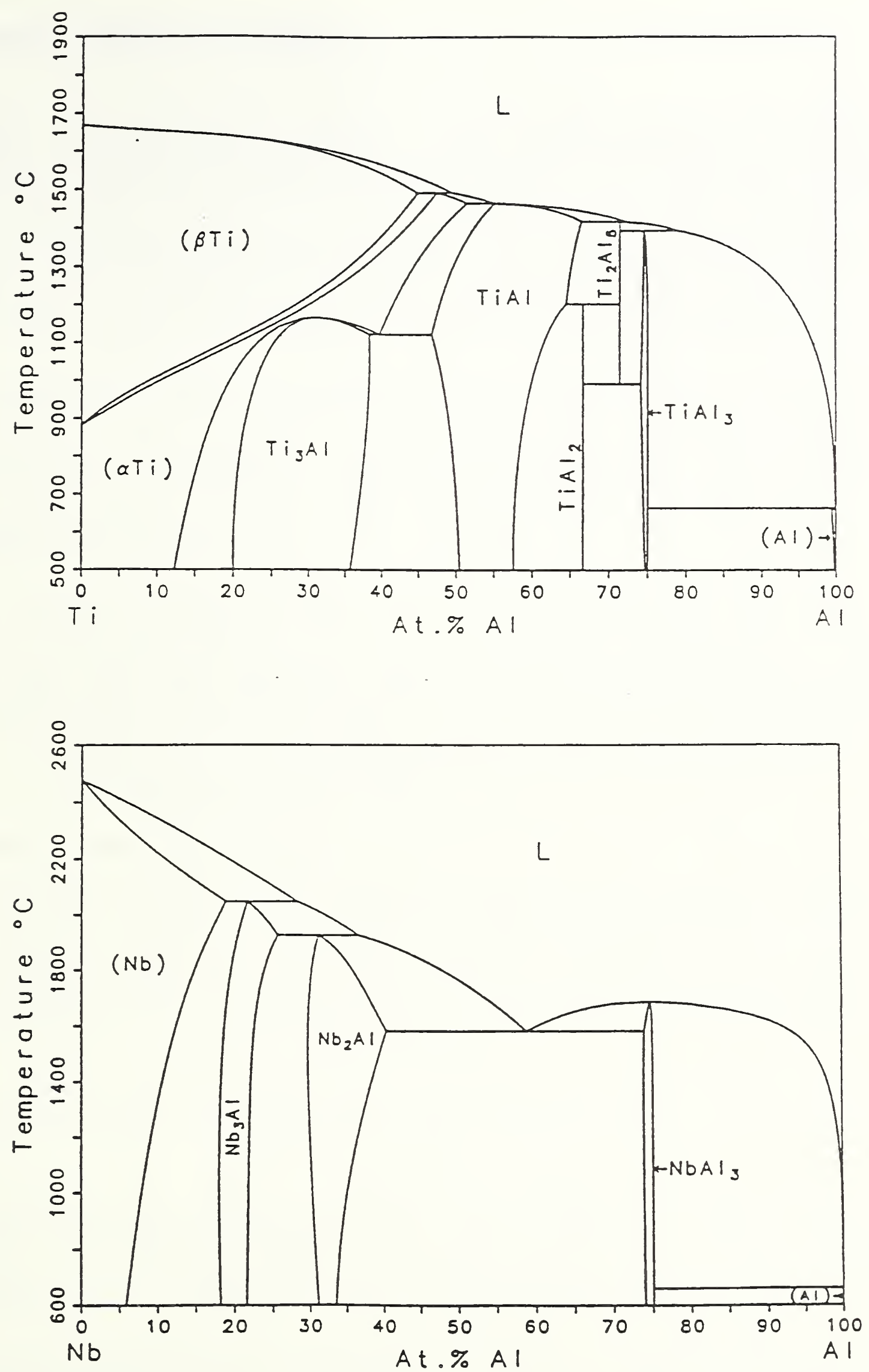

Figure 3. Recent evaluations and thermodynamic calculations done at NIST of binary alloy phase diagrams needed as a basis for determining the Ti-Al-Nb ternary phase diagram. 

In keeping with the principal thrust of the Materials Science and Engineering Laboratory, the Metallurgy Division's program has increasingly turned toward improving the understanding of industrial processes for metals and alloys. The ultimate goal is to help U.S. industry develop real-time, automated systems of process control; so-called intelligent processing systems. In addition to the basic processing equipment, such systems require sensors to measure or monitor important properties or characteristics of a material while it is being processed, automated controls for the processing parameters, and a process model to guide the controls in response to the sensed quantities.

The Metallurgical Sensing and Modeling Group is a major contributor to this effort through its development of nondestructive measurement methodologies with emphasis on the interactions of probing energies with metallurgical characteristics and phenomena. Some of these methodologies furnish the bases for real-time process sensors; others provide the means for gaining better understanding of the processes. This understanding is applied to the development of process models.

The new name of the Group (it had been the Advanced Sensing Group) does not indicate an expanded mission as much as it is intended to more accurately reflect what had already been the Group's activities and expertise. Principal projects this year have centered on the development of eddy-current methods for process sensors, ultrasonic methods for the study of interfaces, and modeling activities related to microstructure evolution in intermetallic alloys. H. Thomas Yolken was Acting Group Leader for the period of November 1, 1990 through July 31, 1991.

\section{FY 91 Significant Accomplishments}

- The Group's eddy current program once again demonstrated the broad and versatile capabilities of this technology for on-line sensing of materials processes. This year's efforts extended previous work on extrusions and powder metallurgy products to the measurement of internal temperatures in rolled aluminum-alloy sheet and to monitoring the pyrolysis of carbon-carbon composites.

- A general theory of eddy-current inversion was developed, which permits determination of the thicknesses of layered materials and has potential application to evaluating the shapes of discrete flaws.

A powerful new ultrasonic technique for studying the interfaces between solid materials was demonstrated. In this technique, guided ultrasonic waves are launched along the interface, and the displacements at the outer surface of the body, due to the acoustic energy radiated from each point on the interface, is imaged with acoustic microscopy.

- Significant progress was achieved in efforts to monitor the position and the microstructure of liquid/solid interfaces during solidification processes. The approach involves the use of ultrasonic waves generated 
and transmitted through the liquid medium in order to avoid grain boundary scattering. Preliminary tests using a new interferometer and a high-power generating laser yielded clear signals from the interface reflections.

Eddy Current Measurement of Temperatures in Aluminum Processing A. H. Kahn and M. L. Mester* * Research Associate, The Aluminum Association; presently at Babcock \& Wilcox

The objective of this project is the development of a non-contact method of measuring the internal temperature of aluminum alloys during processing operations such as rolling and extrusion. Eddy current impedance measurements can determine the electrical resistivity of an alloy by non-contact electromagnetic induction of current in the material. The resistivity, in turn, depends on the temperature of the product. If the temperature dependence of the resistivity of the alloy is known, then eddy current methods can be used to provide measurements of temperature during processing.

A limitation of this approach arises from variations in the composition of the alloys being processed. Corresponding to the allowed range of concentrations of elements within an industry specification for an alloy is an attendant resistivity variation. In turn, this variation will produce a corresponding shift in the temperature reading if no correction is made for the composition variation. The principal problems now being addressed are to determine the basic accuracy of the eddy current system and to establish limits on the apparent temperature shift associated with variations in composition and degree of solid solution of alloying components in commercially specified grades of aluminum.

The eddy current method employed in this period used through-transmission coil geometry in the examination of rolled sheet material. One coil above the sample acted as a driver, inducing currents in the test sample, and a second coil below received a signal influenced by the test material. Measurements of the ratio of the magnitude and phase difference of the receiver voltage relative to the driving current provided the impedance measurements. The measurements were made at $200 \mathrm{~Hz}$, which was approximately equal to the frequency of maximum dissipation of the impressed excitation, the vicinity of greatest sensitivity. It was found empirically that an angle alpha, related to the complex impedance of the sample-coil system, varied linearly with temperature over a range of $200{ }^{\circ} \mathrm{C}$. This linearity provided a calibration of the eddy current system.

A series of tests was performed on samples of aluminum alloy 3004 with eight concentration variations of the elements $\mathrm{Mn}, \mathrm{Mg}$, and $\mathrm{Cu}$. The samples were heated in a furnace to $500{ }^{\circ} \mathrm{C}$ and then transferred to the sensor, which was at room temperature. During the subsequent cooling, readings were recorded by the eddy current sensor and by a thermocouple embedded in each sample. A statistical analysis of the experimental results was conducted by Dr. Eric Lagergren of the Statistical Engineering Division of NIST. For these research samples it was found that the standard deviation of eddy current temperature 
measurements on similar composition specimens was $7.7^{\circ} \mathrm{C}$; the corresponding standard deviation for repeatability on the same specimen was $2.9{ }^{\circ} \mathrm{C}$. Sensitivities to the individual elemental variations also were determined.

The target 1 -sigma variation aimed for in this project was $2{ }^{\circ} \mathrm{C}$. Rather than attempting to do a more controlled laboratory experiment to achieve the desired improvement, NIST investigators and a task group of the Aluminum Association opted for a hot test on long coils of sheet aluminum passed through an annealing furnace, in which the temperature of the product would be steady. Also, the effects of velocity of the product could be studied. The test will be performed by the invitation of Alcan, Ltd. at its annealing facility at Kingston, Ontario. Member companies of the Aluminum Association will supply coils of specified grades and composition ranges for a statistical test of temperature reading variation. Speed and temperature will be varied and thickness will be monitored. Assistance with engineering installation and data collection will be provided by the Data Measurement Corporation (DMC) on contract with the Aluminum Association.

\section{Eddy Current Sensing of Carbon-Carbon Composites During Processing}

A. H. Kahn, M. Gvishi*, and M. L. Mester**

* Guest Scientist - Ministry of Defense, Haifa, Israel

** Research Associate, The Aluminum Association; presently at Babcock \& Wilcox

This project was undertaken to evaluate the feasibility of using an eddy current system for monitoring the pyrolysis of carbon-carbon composite sheets during high temperature processing. Carbon-carbon composite is prepared by heating a graphite fiber, resin impregnated structure until the resin matrix has completely carbonized. Electrical conductivity increases of the order of 100 percent are observed during this heating. Thus, conductivity measurements, particularly by the non-contact eddy current method, are potentially useful for monitoring the processing. This project was cost-shared with the General Dynamics Corporation, Fort Worth, Texas and DARPA.

Using a through-transmission configuration of coils similar to that used for aluminum sheet, but adapted for the higher frequencies needed for the more resistive carbon-carbon, we designed and assembled a high temperature test system. This system operates at fixed frequencies, thereby promising greater precision and more direct interpretation than the pulse method previously used. Flat spiral coils of platinum wire were placed on opposite sides of the test materials. Through-transmission measurements were performed at a series of frequencies ranging from $5 \mathrm{kHz}$ to $2 \mathrm{MHz}$. By comparison of the frequency of peak power absorption with the theoretical prediction of the theory of Dodd and Deeds, appropriately modified for this geometry, the resistivity was found. To check the accuracy, we performed a four-probe van der Pauw DC measurement and obtained agreement within 1.4 percent. Because unprotected carbon-carbon oxidizes at elevated temperatures, this check could be performed only at room temperatures. However, stainless steel sheets offered the possibility of concurrent high temperature eddy current and four-probe DC measurements on a substitute material with the same conductance (conductivity 
$\mathrm{x}$ thickness) as typical carbon-carbon samples. Agreement was excellent up to $500{ }^{\circ} \mathrm{C}$, at which point the stainless steel reacted with air, producing a magnetic oxide that corrupted the AC magnetic eddy current measurement.

Finally, a $15 \times 15 \times 0.25 \mathrm{~cm}$ sheet of fully pyrolyzed carbon-carbon, coated with silicon carbide to prevent oxidation, was put through four heating cycles to $350,575,850$, and $800{ }^{\circ} \mathrm{C}$, with eddy current measurements carried out during heating and cooling. The results indicate that the precision of the conductivity measurements was approximately 2.5 percent over the entire temperature range. In this range the conductivity increased approximately 100 percent. A conductivity model based on contributions of two mechanisms was proposed. On the assumption of temperature-independent conduction by the fibers oriented along the direction of the current, and thermally activated conduction by the semiconducting matrix (as by activated carrier concentration and/or activated mobility), an excellent fit to the data was achieved. The matrix conductivity activation energy was found to be $0.13 \mathrm{eV}$.

The conclusion is that sensing of the resistivity of carbon-carbon sheets by through-transmission eddy current methods at temperatures up to $850^{\circ} \mathrm{C}$ is feasible and practical. In addition, we have proposed a simple mechanism for describing the temperature dependence of the electrical conductivity of carbon-carbon.

General Theory of Eddy-Current Inversion

S. J. Norton and J. R. Bowler*

* University of Surrey, Guildford, U.K.

A general theory of eddy-current inversion has been developed. The eddy-current inversion problem can be described as the task of reconstructing an unknown inhomogeneous distribution of electrical conductivity from impedance measurements recorded as a function of probe position, excitation frequency, or both. In eddy current nondestructive evaluation, this problem is widely recognized as the central theoretical problem whose solution should have important applications in the characterization of inhomogeneities in conducting material. Since the inverse eddy-current problem is nonlinear, an iterative procedure was developed based on a least-squares criterion. At each iteration, the conductivity estimate is modified to bring the predicted impedance data (obtained by solving the forward model) into agreement with the measured impedance data. This results in a reconstruction of the conductivity distribution most consistent with the eddy-current data. Prior information also can be incorporated in a systematic way. The theory also can be applied to the problem of determining the unknown shape, or boundary, of a discrete flaw (void, crack or inclusion).

Thus far, reconstructions based on simulated data have confirmed that the theory gives the correct results for a layered conductor; in particular, the thicknesses and conductivities of the layers have been successfully determined from simulated multi-frequency impedance data. Tests of the theory using experimental impedance data derived from layered systems, and geometrically more complex flaws, also are anticipated. 
Guided Ultrasonic Waves for Acoustic Microscopy of Solid Interfaces E. Drescher-Krasicka and J.A. Simmons

When a guided ultrasonic interface wave travels along the interface between two materials it radiates acoustic energy from each point on the interface, and forms a displacement field at the outer surface of the body. We have found that there is a rich structure of leaky, or evanescent, modes in a clad rod system and that, depending on the particular mode and frequency, the energy leaked by such modes conveys information about the properties of the interface.

Traditional acoustic microscopy uses one acoustic lens working in an echo mode. It usually results in a weak signal from cylindrical interfaces because most of the acoustic energy is scattered by the cylindrical shape of the rod inside the cladding and never comes back to the receiver. In the case of interface waves propagating along an interface, the transmitter excites the interface mode, and the receiver scans the image of the leakage of the acoustic energy through the aluminum cladding. In this application the acoustic lens was used as a receiver focused on the surface of the aluminum cladding where the acoustic signals from the leaky interface mode form the displacement field.

This arrangement of the lenses (transmitter attached to the rod, movable receiver focused at the surface of the sample) was applied to image the discontinuity of the materials at the interface. The model sample, prepared by hot isostatic pressing, consists of a silicon carbide rod with aluminum cladding. Half of the SiC rod was wrapped in a 3-mm thick layer of copper before it was hot pressed in aluminum. The interface wave which propagates on the SiC-Al interface reflects from the discontinuity, and part of the energy converts into a copper-SiC interface mode. This new mode is characterized by a lacuna, the separation in the middle of the image, where part of the acoustic energy starts a new leaky mode (Fig. 1). This mode bounces back and forth at the copper-SiC interface, and has lower attenuation than the Al-SiC mode (right hand side of the image). The best images of the cylindrical interfaces were obtained when the transmitter and the receiver were moving simultaneously, illuminating the interface with the same intensity of acoustic energy. These are among the first images obtained by use of guided interface modes for acoustic microscopy of the interfaces between the two solid materials like metals or a metal and a ceramic.

\section{Ultrasonic Sensing of Liquid/Solid Interfaces in Metals}

W. L. Johnson, J. B. Spicer*, S. J. Norton, and F. Bendec**

* Guest Scientist. Johns Hopkins University

** Guest Scientist - Nuclear Research Center, Negev, Israel

In metallic alloys produced by directional solidification, the mechanical properties of the finished material are greatly influenced by the details of the solidification process. Characterization of the liquid/solid interface is, therefore, of primary importance for both process modeling and process control. This continuing research project has been motivated by the realization that ultrasonic waves constitute one of the few forms of probing energy which can provide real-time information on liquid/solid interfaces in 
metals. Unlike most past research in this area, a major goal of this project has been to obtain information on not just the interface position but also microstructural features of the interface. The very significant scattering of ultrasonic waves from grain boundaries in the solid makes it impossible to obtain information on the microstructure of the interface using ultrasonic waves transmitted through the solid. Therefore, the approach has been to use waves generated and received in the liquid to study ultrasonic scattering from the interface. The focus this year has been on sensor design and basic hardware construction. A variety of transduction configurations has been explored. The most promising configuration uses lasers to generate and receive ultrasonic waves. A transparent rod is immersed in the liquid metal and an ultrasonic pulse is generated directly in the liquid at the end of this rod using an infrared pulse from a Nd-YAG laser which passes through the rod. After the ultrasonic pulse reflects off the liquid/solid metallic interface it is detected at the end of the rod with an interferometer. This configuration was initially tested at Johns Hopkins University in collaboration with $\mathrm{Dr}$. James Wagner and James Spicer. A small pulsed Nd-YAG laser ( $-15 \mathrm{~mJ}$ ) was used for the ultrasonic wave generation and a two-beam interferometer was used for detection. The light was passed through a silica rod which was immersed in liquid tin interfaced to solid brass. A small signal was observed corresponding to reflection off the interface, but detection was complicated by saturation of the interferometer from the generating laser pulse and spurious ultrasonic signals arising from reflections inside the silica rod. The initial test at Johns Hopkins was followed by tests at NIST employing a recently constructed two-beam interferometer and a higher power $150 \mathrm{~mJ}$ Nd-YAG generating laser. The tests were performed with liquid tin interfaced to solid stainless steel. Separate immersed silica rods were used for generation and detection in order to reduce the spurious signals encountered at Johns Hopkins. Clear signals corresponding to the interface reflection were observed.

\section{Composite Solder}

R. B. Clough and R. Patel

Harry Diamond Laboratories is sponsoring a program for producing more reliable and durable solder joints for military hardware. Composite solder can be expected to achieve these goals by increasing the flow stress and decreasing the creep rate. The addition of intermetallic $\mathrm{Cu}_{6} \mathrm{Sn}_{5}$ particulates can also, in principle, suppress growth of the metallic at the copper substrate interface through an equal reduction in the amount of free tin available in the solder matrix. Both of these effects can inhibit the formation and growth of thermal cycle-induced creep fatigue cracks. This study showed that, in fact, very large strengthening (tripling the yield stress) and reduction in creep strain (by a factor of two) is possible through the addition of $30 \mathrm{v} / 0$ intermetallic $\mathrm{Cu}_{6} \mathrm{Sn}_{5}$ particulates. It was, however, impossible if appreciable porosity ( $10 \%$ or more) were present. The porosity in powder solders is produced by residual flux, which coats each particle and is restrained from escaping due to Stokes' Law for particulate (or bubble) motion in viscous fluids. 
Modeling of Microstructure Evolution in Intermetallic Alloys

J. W. Cahn, J. A. Simmons, S. N. Chow*, J. K. Hale* and R. Kikuchi**

* Guest Scientist - Georgia Institute of Technology

** UCLA

The ability to predict metastable phases and the kinetics of microstructure morphology during processing of multicomponent alloy systems is an important need in modern metallurgy. This NIST/DARPA project addresses the formulation of physically realistic modeling equations and the application of emerging solution methods from the mathematical theory of dynamical systems to study the evolution of microstructure in alloy systems with both partial ordering and segregation. The goals of this program include:

- Development of new techniques permitting the rigorous computerized solution of the cluster variation formulation for partially ordered alloy systems. Extension of these techniques to the path probability method for developing a partial differential equation system to serve as a state-of-the-art basis for modeling microstructure evolution in intermetallic alloys.

- Application of new mathematical and numerical algorithms from the dynamical theory of nonlinear partial differential equations to both the qualitative and quantitative solution of equations modeling alloy microstructure evolution.

- Incorporation of global stress and local fluctuation effects into the modeling equations.

- Design and implementation of experiments to critically evaluate theoretical models and their predictions.

- A three-week workshop on Interfacial Pattern Formation at the Aspen Center for Physics was organized to bring together materials scientists, physicists, and mathematicians to deal with the above issues.

The Lyapunov functional for the effect of stress on the free energy takes the form:

$$
F(u, v)=\int f(u, v)+\alpha(\nabla u)^{2}+B(\nabla v)^{2}
$$

where $\mathrm{u}$ is displacement (non-conserved) and $\mathrm{v}$ is concentration (conserved). This form is of the same type as that associated with an ordering reaction, but the stress effects give rise in one dimension to non-local boundary conditions whose consequences are being studied numerically. Work is also in progress on other numerical techniques such as phase field methods for calculating dendritic growth structures in solidification.

A comprehensive overview of nine different mathematical techniques for treating geometric problems of crystal growth with curvature was prepared and a complete three dimensional catalog of interface junctions between four phases with six different isotropic interface boundary energies is being prepared. Experimental verification of some of the predictions of this theory 
are in progress. A Monte Carlo simulation of two-phase grain growth in a twodimensional system with isotropic surfaces was carried out. Also, the possibilities provided by shear transformations--such as deformation twinning and martensitic transformations--for creating unusual ordering structures as initial data for subsequent microstructure evolution during tempering have been explored.

In the area of discrete modeling of the diffusive ordering process, continuation of discrete diffusion problems and a cellular automata technique using a Lyapunov function has been developed. Numerical and graphical outputs of ordering patterns with different stoichiometries and underlying lattice structures are being studied in two dimensional-geometries.

New experimental work is being started in the $\mathrm{Ni}-\mathrm{Al}-\mathrm{V}$ system, which has the advantage over the $\mathrm{Ni}-\mathrm{Al}-\mathrm{Ti}$ system of less sensitivity to oxygen, and the existence of two experimentally accessible types of partial ordering whose kinetic reactions can be studied, possibly in situ. 

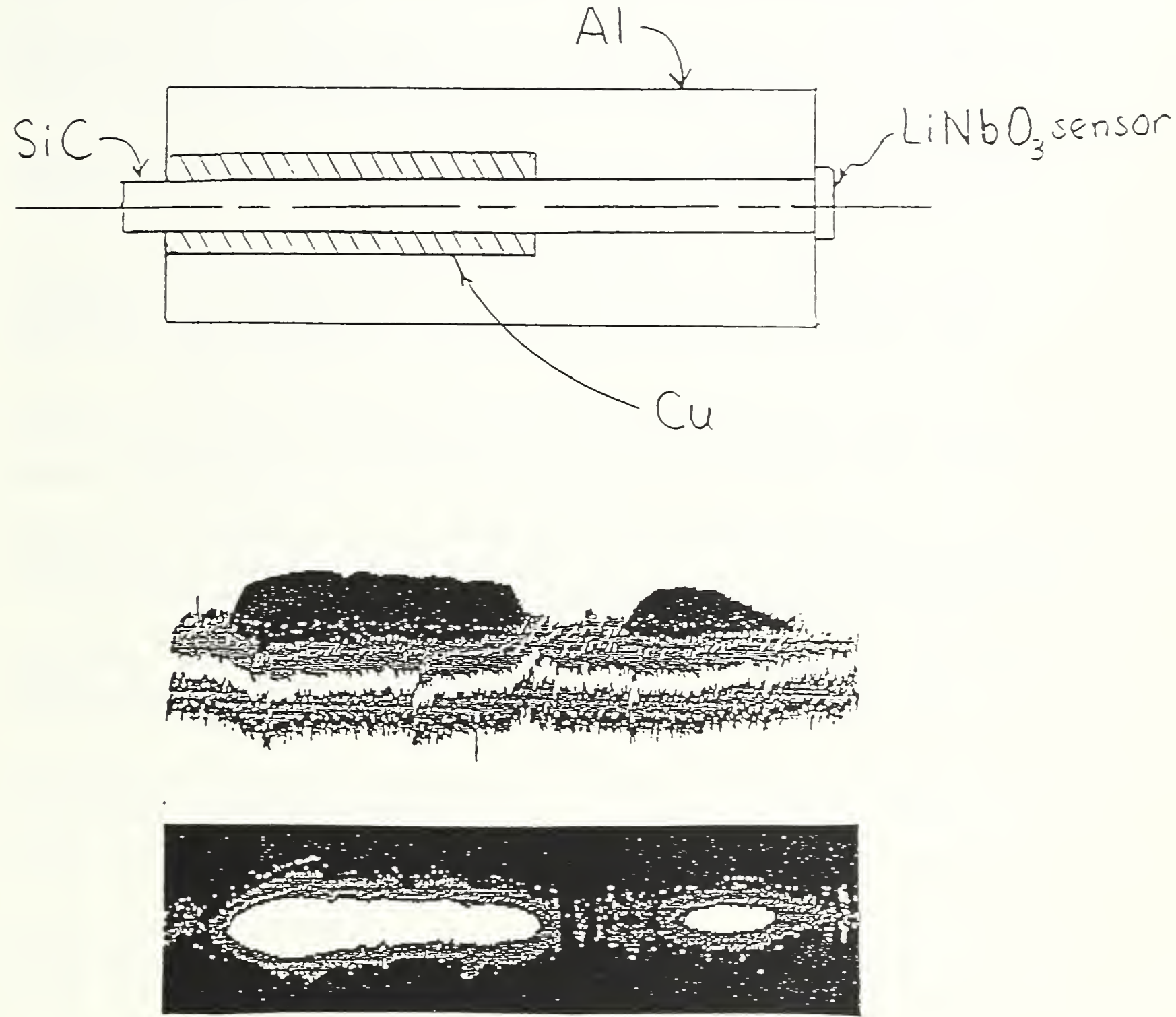

Figure 1 Images of the acoustic energy leaked to the surface from guided ultrasonic waves at the interfaces of a clad metal sample. 



\section{PUBLICATIONS}

\section{Corrosion Group}

Anderson, D. B.; "Evaluation of Corrosion Data", ASTM Symposium on Computer Modeling for Corrosion, (1990).

Anderson, D. B.; "Expert Systems and Material Property Databases", Third International Symposium on Computerization and Uses of Materials Property Data, (1991).

Anderson, D. B.; "NACE-NIST Corrosion Data Program", CINDAS/Purdue DOD Metals Information Analysis Center Newsletter, (1991).

Anderson, D. B.; Lee, T. S.; Syrett, B. C.; "Computerized Corrosion Behavior Information for Materials Selection in FGD Systems", AIRPOL/90, (1990).

Anderson, D. B.; Syrett, B. C.; "POWER-COR Series of Expert Systems and Databases on Corrosion Behavior Information for the Electric Power Industry", EPRI Conference on Expert System Applications for the Electric Power Industry, (1991).

Bertocci, U.; "Statistics of Localized Breakdown Noise," Proceedings of the II $^{\text {nd }}$ International Conference on Localized Corrosion, 127. NACE, Houston, TX (1991).

Bertocci, U.; Ricker, R. E.; "Modeling Polarization Curves and Impedance Spectra for Simple Electrode Systems," accepted by ASTM for publication in the STP to be entitled Computer Modeling for Corrosion, (1991).

Escalante, E.; "Measuring the Corrosion of Metals in Soil." Corrosion Testing and Evaluation, ASTM STP 1000, Am. Soc. for Testing and Materials, Phila. PA (1990).

Escalante, E.; Oka, T.; Bertocci, U.; "Effect of Oxygen Transport and Resistivity of the Environment on the Corrosion of Steel." Materials Research Society Symposium Proceedings, 212, 303, (1991). NIST-IR 904266 (1990).

Fraker, Anna C.; Eichmiller, F.; "Corrosion Principles in Dental Implantology," Clinical Dentistry, J. Hardin, J. B. Lippincot Co. eds, $5,50,1-10,(1991)$.

Interrante, C.; Fraker, A.; Escalante, E.; "Evaluation and Compilation of DOE Waste Package Test Data - Biannual Report." U. S. Nuclear Regulatory Commission, NUREG/CR-4735, 7, in press.

Interrante, C.; Fraker, A.; Escalante, E.; "Evaluation and Compilation of DOE Waste Package Test Data - Biannual Report." U. S. Nuclear Regulatory Commission, NUREG/CR-4735, 8 , in press. 
Interrante, C.; Fraker, A.; Escalante, E.; "Evaluation and Compilation of DOE Waste Package Test Data - Biannual Report." U. S. Nuclear Regulatory Commission, NUREG/CR -4735 , 9, in press.

Interrante, C. G.; Messina, C. A.; Fraker, A. C.; "A Review Process and a Database for Waste-Package Documents," Materials. Research Society Symposium Proceedings, Scientific Basis for Nuclear Waste Management 14, Teafilo A. Abrajano, Jr., and Lawrence H. Johnson, eds. 212, 917-922, (1991).

Interrante, C. G.; Messina, C. A.; Fraker, A. C.; "Information Retrieval Using Key Words and a Structured Review," American Society for Testing and Materials (ASTM) Special Technical Publication Standardization of Terminology, ed. R. Strehlow, in press.

Ricker, R. E.; Bertocci, U.; Fink, J. L., Stoudt, M. R.; "Evaluation of the Environmental Induced Fracture Resistance of Ductile Nickel Aluminide," Environmental Effects on Advanced Materials, 213. TMS, Warrendale, PA. R. H. Jones and R. E. Ricker eds, TMS, (1991).

Ricker, R. E.; Fink, J. L.; and Vasudevan, A. K.; "On the Stress Corrosion Cracking of Al-Li Alloys: The Role of Grain Boundary Precipitates," Metal1. Trans. A, 22A, 1, 264-267 (1991).

Ricker, R. E.; Jones, R. H.; "Mechanisms of Stress-Corrosion Cracking" to be published in Stress-Corrosion Cracking: Materials Performance and Evaluation by ASM International, Materials Park, OH. (1991)

Stoudt, M. R.; Escalante, E., Ricker, R. E.; "The Influence of Moisture and Pressure on the Mechanical Properties of a Glass-Epoxy Matrix Composite and a Graphite-Epoxy Matrix Composite," submitted to the National Association of Corrosion Engineers for publication in Environmental Effects in Advanced Materials, (1991).

Stoudt, M. R.; Escalante, E., Ricker, R. E.; "The Influence of Water on the Mechanical Properties of a Glass-Epoxy Matrix Composite," Advanced Composite Materials, Ceramic Transactions, 19, M. D. Sacks, ed., ACerS Press (1991).

Stoudt, M. R., Vasudevan, A. K., Ricker, R. E.; "Examination of the Influence of Lithium on the Repassivation Rate of Aluminum Alloys," Corrosion Testing of Aluminum Alloys, ASTM, STP $1134 \mathrm{~V}$. Agarwala ed., Am. Soc. for Testing and Mats, Phila, PA 196-213 (1991).

Sturrock, C.P.; "Knowledge Engineering of Materials Advisory Systems", 3rd International Symposium on the Computerization and Use of Materials Property Data, American Society for Testing and Materials, Philadelphia, PA, (1991).

Sturrock, C.P.; "The Development of Non-proprietary Information Systems for Expert and Non-specialist Users", World Congress on Expert Systems, in press. 
Sturrock, C.P.; Anderson, D.B.; Krisher, A.S.; Lee, T. S.; "Corrosion Evaluation Information Dissemination via Computers," in Corrosion

Testing and Evaluation: Silver Anniversary Volume. ASTM STP 1000, R. Baboian and S.W. Dean, eds., American Society for Testing and Materials, Philadelphia, PA, (1990).

\section{Electrodeposition Group}

Grushko, B.; Stafford, G. R.; "Phase Formation in Electrodeposited and Thermally Annealed Al-Mn Alloys." Met. Trans. 21A, 2869-2879 (1990).

Janowski, G. M.; Stafford, G. R.; "The Microstructure of Electrodeposited Titanium-Aluminum Alloys." Met. Trans., in press.

Lashmore, D. S.; "The Metallurgy of Nickel Phosphorus Alloys." ASPE Spring Meeting Metal Platings for Precision Finishing Operations, (1991).

Stafford, G. R.; Cahen, G. L.; Stoner, G. E.; "Graphite Fiber-Polymer Matrix Composites as Electrolysis Electrodes." J. Electrochem. Soc. $138,425-430$ (1991).

Wheeler, N. S.; "Review of the Nickel-Grpahite Interface." J. of Composites Technology and Research, 12, (3), 177-183 (1990).

Wheeler, N. S.; Lashmore, D. S.; Shapiro, A. J.; Handwerker, C. A.; "Structural Chracterization of the Nickel-Graphite Interface." submitted (1991).

\section{High Temperature Materials Chemistry Group}

Brody, P. S.; Benedetto, J. M.; Rod, B. J.; Benett, K. W.; Cook, I. P.; Schenck, P. K.; Chiang, C. K.; Wong-Ng, W.; "Microstructure and Ferroelectric Properties of Lead Zirconate-Titanate Films Produced by Laser Evaporation," Proc. of The 1990 International Symposium on Ferroelectrics, (1990)

Chiang, C. K.; Cook, L. P.; Schenck, P. K.; Brody, P. S.; Benedetto, J . M.; "Characterization of Lead Zirconate-Titanate Thin Films Prepared by the Laser Ablation Technique," Materials Research Society Symposium Proceedings Series, 200, Spring MRS Meeting, Symposium Y: Ferroelectric Thin Films, (1990)

Cook, L. P.; Vaudin, M. D.; Schenck, P. K.; Wong-Ng, W.; Chiang, C. K. ; and Brody, P. S.; "Microstructural Changes During Processing of LaserDeposited $\mathrm{BaTiO}_{3}$ and PZT Thin Films," (Materials Research Society, 1990)

Cook, L. P.; and Plante, E. R.; "Phase Diagram of the System $\mathrm{Li}_{2} \mathrm{O}$ $\mathrm{Al}_{2} \mathrm{O}_{3}$," submitted to Lithium Ceramics III, ed. by G. Hollenberg and I. Hastings, (American Ceramic Society, 1991) 
Hastie, J. W.; Bonnell, D. W.; and Berkowitz, J.; "High Temperature," in The Encyclopedia of Physics, 2nd Edition (American Institute of Physics, VCH Publishers, Inc. 1991)

Hastie, J. W.; and Bonne11, D. W.; eds.; "Proc. 6th Int. Conf. High Temperatures--Chemistry of Inorganic Materials," in High Temperature Science, vols. 26, 27, 28 (1990)

Kowalska, M.; Plante, E. R.; and Hastie, J. W.; "Activity of FeO in the $\mathrm{FeO}-\mathrm{MgO}-\mathrm{SiO}_{2}$ System, Determined by High Temperature Mass Spectrometry," To be submitted to Acta. Metall. (1991)

Schenck, P. K.; Bonne11, D. W. ; Hastie, J. W. ; Cook, L. P.; and Chiang, C. K.; "Optical Characterization of Thin Film Laser Deposition Processes," SPIE Proc. of 1991 Technical Symposium on Microelectronic Processing Integration, (San Jose, CA, 1991)

\section{Magnetic Materials}

Aviram, I., Bennett, L. H., Swartzendruber, L. J., Satija, I. I., "Complex Dynamics in the Barkhausen Effect", JMMM 92, 92, (1991).

Bennett, L. H., "Flux Pinning in High-Temperature Superconductors" Fifth Israel Materials Engineering Conference I.M.E.C.V. (1990).

Bennett, L. H., McMichael, R. D., Swartzendruber, L. J., Shull, R. D., Watson, R. E., "Monte Carlo and Mean Field Calculations of the Magnetocaloric Effect of Ferromagnetically Interacting Clusters", JMMM (in press) (1991).

Bennett, L. H., Swartzendruber, L. J., Atzomy U., Lashmore, D. S., Abdul-Razzaq, W. "Magnetic Aftereffect in Compositionallymodulated $\mathrm{Ni} / \mathrm{Cu}$ Multilayers Prepared by Electrodeposition and by Sputtering", Mat. Res. Soc. Symp. Proc. 160, (1990).

Bennett, L. H., Swartzendruber, L. J., Ettedgui, H., Atzmony, U., Lashmore, D. S., "Magnetic Viscosity in $\mathrm{Ni} / \mathrm{Cu}$ Compositionally Modulated Alloys", J. Appl. Phys. 67, 9, (1990).

Bennett, L. H., Swartzendruber, L. J., Kaiser, D. L. , "Meissner, Shielding, and Flux Loss Behavior in Single-Crystal $\mathrm{YBa}_{2} \mathrm{Cu}_{3} \mathrm{O}_{6+x}$ ", J. App1. Phys. 69, 8, (1991).

Bennett, L. H., Swartzendruber, L. J., Kaiser, D., Bohandy, J., Kim, B. F., Adrian, F. J., Moorjani, K., "SQUID and Magnetically Modulated Microwave Absorption Observations of the Superconducting Transition in Single Crystals of $\mathrm{YBa}_{2} \mathrm{Cu}_{3} \mathrm{O}_{\mathrm{x}}$ ", J. App1. Phys. 67, 9, (1990).

Bennett, L. H., Swartzenduber, L. J., Kaiser, D. L., Gayle, F. W., Blendell, J., Habib, J. M. Seyoun, H. M., "Thermoremanance and 
Meissner Effect in QMG and Single Crystal YBCO" Edinburgh

International Conference on Magnetism [ICM], JMMM (1991).

Bennett, L. H., Turchinskaya, M., Swartzendruber, L. J., Roitburd, A., Lundy, D., Ritter, J., Kaiser, D. L., "Flux Flow and Flux Dynamics in High- $\mathrm{T}_{\mathrm{C}}$ Superconductors", Proceedings AMSAHTS 1990 (NASA Conference Publication 3100) 213, (1990).

Bennett, L. H., Swartzendruber, L. J., Aviram, Satija, I. I., "Complex Dynamics in Domain Wall Motion", J. Appl. Phys. 67, 9, (1990).

Chiang, C., K., Turchinskaya, M., Swartzendruber, L. J., Shull, R. D., Bennett, L. H., "Levitation of Superconducting Composites", AMSAHTS 90: Advances in Materials Science and Applications of High Temperature Superconductors, Proceedings AMSAHTS (NASA Conference Publication 3100), 181, (1990).

Chiang, C. K., Wong-Ng, W., Cook, L. P., Freiman, S. W., Hwang, N. N. M., Vaudin, M., Hill, M. D., "Processing Bi-Pb-Sr-Ca-Cu-O Superconductors From Amorphous State", Proceedings AMSAHTS (NASA Conference Publication 3100), 127, (1990).

Cook, L. P., Chiang, C. K., Wong-Ng, Swartzendruber, L. J . Swartzendruber, Bennett, L. H., "Phase Equilibria in the jSystem Tl-Ca-Ba-Cu-O. I. Stability of the 2122 Phase Under Conditions of Oxygen Annealing", Mat. Res. Soc. Symp. Proc., 169, Materials Research Society, 137, (1990).

Kaiser, D. L., Gayle, F. W., Swartzendruber, L. J. and Bennett, L. $\mathrm{H}$. , "Effect of Twin Boundaries on Flux Pinning in $\mathrm{YBa}_{2} \mathrm{Cu}_{3} \mathrm{O}_{7-x}$ At Low and Intermediate Magnetic Fields" J. of Appl. Phys. (in press).

Kaiser, D. L., Swartzendruber, L. J., Gayle, F. W., Bennett, L. $\mathrm{H}$. , "Lower Critical Field Measurements in $\mathrm{YBa}_{2} \mathrm{Cu}_{3} \mathrm{O}_{6+x}$ Single Crystals" Proceedings AMSAHTS (NASA Conference Publication 3100), 249, (1990).

Lashmore, D. S., Oberle, R. Moshe, P. D., Bennett, L. H., Swartzendruber, L. J., "Electrodeposited Metallic Superlattices", Mat. Res. Soc. Symp. Proc., 132, 219, (1989).

Lashmore, D. S., Oberle, R. R., Bennett, L. H., Swartzendruber, L. J., Atzmony, U., Dariel M. P., Romankiw, "Magnetic and Structural Properties of Electrodeposited Copper-Nickel Microlayered Alloys" Proceedings of the Symp. On Magnetic Materials, Processes, and Devices, Proceedings, 90-8, 347, (1990).

McMichael, R. D., Shull, R. D., Swartzendruber, L. J., Bennett, L. H., Watson, R. E. "Magnetocaloric Effect in Superparamagnets" submitted to the JMMM 1991. 
Roitburd, A., Swartzendruber, L. J., Kaiser, D. L., Gayle, F. W., Bennett, L. H., "Temperature Dependence of Vortex-Twin-Boundary Interaction in $\mathrm{YBa}_{2} \mathrm{Cu}_{3} \mathrm{O}_{6+x} "$, Phys. Rev. Lett., 64, (24), 2962, (1990).

Roytburd, A. L, Turchinskaya, M. J., "Vortex Movement and Magnetization of High Tc Superconductors", Proceedings AMSAHTS (NASA Conference Publication 3100), 203, 1990.

Seyoum, H. M., Habib, J. M., Bennett, L. H., Wong-Ng, W. Shapiro, A. J., and Swartzendruber, L. J., " Superconducting Properties of $\mathrm{Bi}_{2-x-y} \mathrm{~Pb}_{x} \mathrm{Sn}_{y} \mathrm{Sr}_{2} \mathrm{Ca}_{2} \mathrm{Cu}_{3} \mathrm{O}_{z} "$ Printed in the UK, Spercond. Sci. Technol. $\underline{3}, 616$, (1990).

Shull, R. D., (Japan Technology Program) "High Magnetic Field Facilities in Japan Related to Superconductivity Research", NIST Publ. NISTIR 4593, (1991).

Shull, R. D., Bennett, L. H., "Nanocomposite Magnetic Materials", submitted to Acta Metallurgica (1990).

Shull, R. D., Cline, J. P., "High Temperature X-Ray Diffractometry of Ti-Al Alloys", High Temperature Science, $\underline{26}$, by the Humana Press Inc., (1990).

Shull, R. D., Ritter, J. J., "Spin Glass Magnetic Behavior of Iron/Silica Gel Nanocomposites", Mat. Res. Soc. Symp. Proc. 195, (1990).

Shull, R. D., Ritter, J. J., Shapiro, A. J., Swartzenduber, L. J., Bennett, L. H., "Iron Magnetic Moments in Iron/Silica Gel Nanocomposites" J. Appl. Phys. 67 (9), (1990).

Shull, R. D., Ritter, J. J., Shapiro, A. J., Swartzendruber, L. J., Bennett, L. H., "Magnetic Behavior of Nanocomposites Prepared in a Vitreous Alumina Gel", Mat. Res. Soc. Sym. Proc., 206, (1991).

Shull, R. D., Ritter, J. J., Swartzendruber, L. H., "Change in Magnetic State of Fe+Silica Gel Nanocomposites Due to Low Temperature Treatment in Ammonia, J. Appl. Phys. 69 (8), (1991).

Shu11, R. D., Swartzendruber, L. J., Bennett, L. H., "The Magnetocaloric Effect in Nanocomposites" Published in Proceedings of the Sixth International Cryocoolers Conference, Edited by $G$. Green \& M. Knox, David Taylor Research Center Publ. 非DRC-91/002, Annapolis, MD, 231, (1991).

Swartzendruber, L. J., Bennett, L. H., Ettedgui, H., Aviram, I., "Barkhausen Jump Correlations in Thin Foils of $\mathrm{Fe}$ and $\mathrm{Ni}$, J. Appl. Phys. 67 (9), (1990). 
Swartzendruber, L. J., Kaiser, D. L., Bennett, L. H., Gayle, F. W., Roytburd, A., "Low Field Flux Pinning in Twinned and Detwinned Single Crystals $\mathrm{YBa}_{2} \mathrm{Cu}_{3} \mathrm{O}_{7} "$, Appl. Phys. Lett 58 (14), (1991).

Swartzendruber, L J., Roitburd, A., Kaiser, D. L., Gayle, F. W., Bennett, L. H., "Direct Evidenct for an Effect of Twin Boundaries on Flux Pinning in Single-Crystal $\mathrm{YBa}_{2} \mathrm{Cu}_{3} \mathrm{O}_{6+x}$ ", Phys. Rev. Lett. $\underline{64}, 483(1990)$.

Rubinstein, M., Swartzendruber, L. J., Bennett, L. H., Ritter, J. $\mathrm{J}$. Atzomy, U., "Fe Mössbauer Effect in $\mathrm{Y}_{\mathrm{x}} \mathrm{Pr}_{1-\mathrm{x}} \mathrm{Ba}_{2}\left(\mathrm{Cu}_{0.98} \mathrm{Fe}_{0.02}\right)_{3} \mathrm{O}_{7}$ " J . Appl. Phys. 67 (9), (1990).

Turchinskaya, M. J., Roytburd, A. I., Bennett, L. H., Swartzendruber, L. J., and Sawano, K., "Determination of Thermoactivation Parameters of Vortex Mobility in $\mathrm{YBa}_{2} \mathrm{Cu}_{3} \mathrm{O}_{7}$ Using Only Magnetic Measurements" Submitted to Physica C (1990).

Turchinskaya, M., Bennett, I. H., Swartzendruber, L. J., Roitburd, A., Chiang, C. K., Hill, M., Blendell, J. E., Sawano, K., "Comparison of Flux Dynamics in Two Samples of $\mathrm{YBa}_{2} \mathrm{Cu}_{3} \mathrm{O}_{7}$ With Different Pinning", Mat. Res. Soc. Symp. Proc., 169, (1990).

Turchinskaya, M. J., Roytburd, A. J., Bennett, L. H., Swartzendurber, I. J., Sawano, K., "Determination of Thermoactivation Parameters of Vortex Mobility in $\mathrm{YBa}_{2} \mathrm{Cu}_{3} \mathrm{O}_{7}$ Using Only Magnetic Measurements", Physica-C (in press) (1991).

Vajda, F., Oti, J., Pardavi-Horvath, M., Della Torre, E., Swartzendruber, L. H., Bennett, L. H., "Experimental Verification of a Vector Preisach Model", MMM San Diego, CA, submitted to J. of Appl. Phys. (1990).

Watson, R. E., Bennett, I. H., "Transition Metal/Metalloid Crystalline and Glassy Phases" submitted to Physical Review (1990).

Watson, R. E., Bennett, L. H., "Crystalline and Glassy Phases of Transition-Metal - Metalloid Systems", Phys. Rev. B 43(14), 11, 642 (1991).

\section{Mechanical Processing and Performance Group}

Birnbaum, G.; Eitzen, D. G.; and Mordfin, L.; "Recent Developments in NDE Standards at NIST, " Nondestructive Testing Standards II: New Opportunities for Increased World Trade Through Accepted Standards for NDT and Quality, ASTM Special Technical Publication (in press).

dewit, R.; Fields, R. J.; and Irwin, G. R.; "Use of Thickness Reduction to Estimate Fracture Toughness," Constraint Effects in Fracture (ASTM Special Technical Publication, in press). 
Fields, B. A.; and Fields, R. J.; "The Prediction of Elevated Temperature Deformation of Structural Steel Under Anisothermal Conditions," NISTIR 4497 (Jan. 1991).

Fields, R. J.; Low, III, S. R.; Lucey, Jr., G. K.; "Some Properties of Intermetallic Compounds Commonly Found in Solder Joints," Metal Science of Joining (Proceedings of TMS Symposium, Cincinnati, OH, Oct. 20-24, 1991, in press).

Hicho, G. E.; "Crack Arrest Fracture Toughness Measurements of Normalized and Inclusion Shape Controlled AAR TC128 Grade B Steel, and Micro-Alloyed, Control-Rolled, and Inclusion Shape Controlled A 8XX Grade B Steel," NISTIR 4501 (FRA Report No. 21, Feb. 1991).

Hicho, G. E.; Boettinger, W. J.; Swartzendruber, L.; and Shives, T. R.; "Examination of the Excessive Retained Austenite on the Surface of a Section of 17-7 Precipitation Hardening Stainless Steel," NISTIR 4502 (Jan. 1991).

Hicho, G. E.; and Harne, D. E.; " Mechanical Properties and Fracture Toughness of AAR TC128 Grade B Steel in the Normalized, and Normalized and Stress Relieved Conditions," NISTIR (in press).

Hicho, G. E.; Smith, L. C.; Handwerker, C. A.; and Kauffman, D. A.; " Determination of the Prior Austenitic Grain Size of Selected Steels Using a Molten Glass Etch," ASM Journal of Heat Treating (in press).

Low, III, S. R.; Fields, R. J.; and Lucey, Jr., G. K. ; "Multiaxial Mechanical Behavior of $63 \mathrm{Sn}-37 \mathrm{~Pb}$ Solder," Proceedings, 4lst Electronic Components and Technology Conference (Atlanta, GA, May 13-15, 1991), 292-298 (1991).

Low, III, S. R.; Harne, D. E.; and Fields, R. J.; "Dynamic Strength Tests on Electrical Cables Specified for Aircraft Landing Structures," Advanced Technology in Failure Prevention (Proceedings, $43 \mathrm{rd}$ Meeting of the Mechanical Failures Prevention Group), 32-43 (Cambridge University Press, 1991).

Mordfin, L.; "Accumulation of Creep Damage under Varying Temperature Conditions," Proceedings, 6th International Conference on Mechanical Behaviour of Materials (Kyoto, Japan, July 28-Aug. 2, 1991), Vol. 4, 565-570 (1991).

Mordfin, L.; "U.S. Participation in the Development of International Standards for Nondestructive Testing," Nondestructive Testing Standards II: New Opportunities for Increased World Trade Through Accepted Standards for NDT and Quality, ASTM Special Technical Publication (in press). 
Naus, D. J.; Keeney-Walker, J.; Bass, B. R.; Fields, R. J.; deWit, R.; and Low, III, S. R.; "Wide-Plate Crack-Arrest Tests Utilizing Prototypical and Degraded (Simulated) Pressure Vessel Steels, " Nuclear Engineering and Design (in press).

Shives, T. R., editor; Advanced Technology in Failure Prevention (Proceedings, 43rd Meeting of the Mechanical Failures Prevention Group), 258 pp (Cambridge University Press, 1991).

Shives, T. R.; and Fields, R. J.; "Elastic Modulus and Poisson's Ratio of 17-4 PH and 410 Stainless Steels," NISTIR (submitted to WERB).

Shives, T. R.; and Smith, J. H.; "Intercomparison Study of Rockwell Hardness Test Blocks," NISTIR 4531 (Feb. 1991).

\section{Metallurgical Processing Group}

Aprigliano, L. F.; Green, G.; Chafe, J.; O'Connor, L.; Biancaniello, F.; and Ridder, S.; "Development of Neodymium and $E r_{3} N i$ Regenerator Materials", submitted for publication in proceedings of the International Cryogenic Materials Conference, Huntsville, Alabama, (June $11-14,1991$ ).

Basu, B.; Sekhar, J. A.; Schaefer, R. J.; and Mehrabian, R.; "Analysis of the Steady State Molten Pool Obtained by Heating a Substrate with an Electron Beam," Acta Metall. Mater. 39, 725-733 (1991).

Bendersky, L. A.; Boettinger, W. J.; and Roytburd, A.; "Coherent Precipitates in the BCC/Orthorhombic Two Phase Field of the Ti-Al-Nb System." Acta Met. 39, 1957-1969 (1991).

Bendersky, L. A.; Boettinger, W. J., and Roytburd, A.; "Crystallography and Thermodynamics of Heirarchical Structures of ( $\mathrm{Ti}, \mathrm{Nb})_{3} \mathrm{Al}$ Alloys", in Proceedings of International Symposium on Intermetallic Compounds Structure and Properties - (JIMIS-6), ed. by 0. Izumi, The Japan Institute of Metals, Sendai, Japan, 1991, pp. 845-849.

Bendersky, L. A.; Ridder, S. D.; Biancaniello, F. S.; and Shapiro, A. J.; "Microstructural Characterization of Atomized Powder of Al-5Mn5Fe-2Si (wt\%) Alloy." Proceedings of the 7 th International Conference on Rapidly Quenched Materials, Mat. Sci. and Eng., A134, (1991).

Bendersky, L. A.; Roth, R. S.; Ramon, J. J. and Schectman D. "Crystallographic Characterization of Some Intermetallic Compounds in the Al-Cr System", Met. Trans. 22A, 5 (1991).

Bendersky, L. A.; Burton, B. P., Boettinger and Biancaniello F. S.; "Ordered $\omega$-derivatives in a Ti-37.5Al-20Nb at \% Alloy, Scr. Met., 24, 1541 (1990). 
Bennett, L. H.; Swartzendruber, L.; Kaiser, D. L. ; Gayle, F. W.; Blendell, J.; Habib, J. M.; and Seyoum, H. M.; "Thermoremanance and Meissner Effect in QMG and Single Crystal YBCO," accepted for publication, Journal of Magnetism and Magnetic Materials (1991).

Boettinger, W. J.; Bendersky, L. A.; Aziz, M. J.; Cline, J.; and West, J. A.; "Disorder Trapping $\mathrm{Ni}_{2}$ TiAl." Mat. Sci. and Eng. Al33 (1991), $592-595$.

Boettinger, W. J.; Bendersky, L. A.; Mozer, B.; and Rowe, R. G.; "Neutron Powder Diffraction Study of the Orthorhombic Ti ${ }_{2} \mathrm{AlNb}$ Phase." Scripta Met. 24 (1990), 2363-2368.

Boettinger, W. J.; Thermodynamic Constraints on Non-Equilibrium Solidification of Ordered Intermetallic Compounds. Mat. Sci. \& Eng. A133 (1991), 438-442.

Boettinger, W. J.; Handwerker, C. A. and Smith, L. C. in "On the Wetting of the Intermetallics $\mathrm{Cu}_{6} \mathrm{Sn}_{5}$ and $\mathrm{Cu}_{3} \mathrm{Sn}$ by $\mathrm{Pb}-\mathrm{Sn}$ Alloys," in The Metal Science of Joining, eds. M. J. Cieslak, M. E. Glicksman, and J. H. Perpezko, TMS-AIME, Warrendale, PA, submitted.

Boettinger, W. J.; Shapiro, A. J.; Cline, J. P.; Gayle, F. W.; Bendersky L. A.; and Biancaniello, F. S.; "Investigation of the Phase Constitution of $\mathrm{Al}_{2} \mathrm{TiTa}$, Scripta Met. Mat., 25 (1991), 1993-1998.

Brush, L. N., McFadden, G. B., Coriell, S. R., "Laser Melting of Thin Silicon Films, J. Crystal Growth, in press.

Burton, B. P.; Osburn, J. E.; and Pasturel, A.; "Theoretical Calculations of the NiAl-NiTi Phase Diagram Based LMTO and LAPW Cohesive Energy Calculations". Accepted for publication in Phys. Rev. B 7/91.

Burton, B. P.; Pasture1, A.; and Carter, W. C.; (1991) LMTO/CVM Calculations of Partial BCC based Phase Relations in the System Ni-AlTi. Proceedings of the International Workshop on Ordering and Disordering in alloys, Grenoble (1991).

Burton, B. P.; "The Interplay of Chemical and Magnetic Ordering in Oxide Minerals". Reviews in Mineralogy V25, 303-321, D.H. Lindsley Ed. MSA, Wash. DC. (1991)

Burton, B. P.; Osburn, J. E.; and Pasturel A.; "LMTO/CVM and LAPW/CVM Calculations of the NiAl-NiTi Pseudobinary Phase Diagram". Fall 1990 MRS Symposium on High-Temperature Ordered Intermetallic Alloys, IV, V213, 107-112, L. A. Johnson, D. P. Pope, J. O. Stiegler, Editors.

Burton, B. P.; "The System Fe-Pb". (1991) Journal of Phase Equilibria, V12-2, 200-202 (1991). 
Cahn, J. W.; Taylor, J. E.; and Handwerker, C. A.; "Evolving Crystal Forms: Frank's Characteristics Revisited." Submitted to Sir Charles Frank 80 th Birthday Tribute, Ed. R. G. Chambers, J. E. Enderby, A. Keller, A. R. Lang, and J. W. Steeds, Adam Hilger Publishers, Bristol, (1991).

Coriell, S. R., and McFadden, G. B., "Instability during Directional Solidification: Gravational Effects, in Low-Gravity Fluid Dynamics and Transport Phenomena, ed. Koster, J. N. and Sani, R. L., (American Institute of Aeronautics and Astronautics, Washington, 1990).

Gayle, F. W.; Shapiro, A. J.;Biancaniello, F. S; and Boettinger, W. J.; "The Al-Cu-Fe Phase Diagram - Equilibria Involving The Icosahedral Phase", Met. Trans. A, submitted.

Gayle, F. W.; and Kaiser, D. L.; "The Nature of [001] Tilt Grain Boundaries in $\mathrm{YBa}_{2} \mathrm{Cu}_{3} \mathrm{O}_{6+\mathrm{x}}$." Journal of Materials Research 6, 1991, 908 915.

Goldman, A. I.; Stassis, C.; Bellissent, R.; Moudden, H.; Pyka, J.; and Gayle, F. W.; "Inelastic Neutron Scattering Measurements of Phonons in Icosahedral Al-Li-Cu." Physical Review B43, 1991, 8763-8766.

Handwerker, C. A.; Blendell, J. E.; Coble, R. L.; "Sintering of Ceramics," Science of Sintering, Ed. D.P. Uskokovic, H. Palmour, and R. M. Spriggs, Plenum Press, New York, (1990).

Handwerker, C. A.; Lechtman, H. N.; Marinenko, R.; Bright, D. S.; and Newbury, D. N.; "Fabrication of Pt-Au Alloys in Pre-Hispanic South America: Issues of Temperature and Microstructure Control," MRS Conference Proceedings Series, Volume 185, Ed. P. B. Vandiver, J. Druzik, and G. S. Wheeler, (1991).

Hardy, S. C., McFadden, G. B., Coriell, S. R., Voorhees, P. W., and Sekerka, R. F., "Measurement and Analysis of Grain Boundary Grooving by Volume Diffusion", J. Crystal Growth, in press.

Hicho, G. E.; Smith, L. C.; Handwerker, C. A.; and Kauffman, D. A.; "Determination of the Prior-Austenitic Grain Size of Selected Steels using a Molton Glass Etch." J. Heat Treating, 9, 37-47 (1991).

Kaiser, D. L. ; Gayle, F. W.; Swartzendruber, L. J.; Wong-Ng, W.; Watkins, S. F.; and Fronczek, F. R.; "Structural and Magnetic Properties of Untwinned $\mathrm{YBa}_{2} \mathrm{Cu}_{3} \mathrm{O}_{6+x}$ Single Crystals." In: $\underline{\mathrm{H} i g h}$ Temperature Superconductors: Fundamental Properties and Novel Materials Processing, Materials Research Society, Symp. Proc. 169, 1990, 793-796. 
Kaiser, D. L. ; Swartzendruber, L. J. ; Gayle, F. W. ; and Bennett, L. H. ; "Lower Critical Field Measurements in $\mathrm{YBa}_{2} \mathrm{Cu}_{3} \mathrm{O}_{6+x}$ Single Crystals." In: AMSAHTS'90: Advances in Materials Science and Applications of High

Temperature Superconductors, Bennett, L. H., Flom, Y., and Moorjani, K. (eds.), NASA Conference Publication 3100, Washington, D.C., 1991, 249 255 .

Kattner, U. R. The Ta-Al (Tantalum-Aluminum) System: Binary Alloy Phase Diagrams (2nd edition), ASM International, Materials Park, Ohio, Dec. 1990.

Kattner, U. R. The Nb-Al (Niobium-Aluminum) System: Binary Alloy Phase Diagrams (2nd edition), ASM International, Materials Park, Ohio, Dec. 1990.

Kattner, U. R. The Ga-Te (Gallium-Tellurium) System: Binary Alloy Phase Diagrams (2nd edition), ASM International, Materials Park, Ohio, Dec. 1990.

Kattner, U. R. The Fe-Al [Iron-Aluminum] System: Binary Alloy Phase Diagrams (2nd edition). ASM International, Materials Park, Ohio, Dec. 1990.

Kaiser, D. F.; Gayle, F. W., Swartzendruber, L. J.; Bennett, L. H.; and McMichael, R. D.; "Effect of Twin Boundaries on Flux Pinning in $\mathrm{YBa}_{2} \mathrm{Cu}_{3} \mathrm{O}_{7-x}$ at Low and Intermediate Magnetic Fields," J. Applied Physics, accepted for publication.

Lucey, G.; Marshall, J.; Handwerker, C. A.; Tench, D.; and Sunwoo, A. "A Hypothesis for the Mechanism of Solderability Failure," NEPCON '91 West Conference Proceedings, Cahners Exposition Group, DesPlaines, IL, (1991).

Marinenko, R. B.; Bright, D. S.; Handwerker, C. A. ; and Mecholsky, Jr., J. J.; "A Study of Diffusion Zones with Electron Microprobe Compositional Mapping," Microbeam Analysis - 1990, Ed. J. R. Michael and Peter Ingram, San Francisco Press (1990).

Marinenko, R. B.; Bright, D. S.; Handwerker, C. A.; and Mecholsky, Jr., J. J.; "A Study of Diffusion Zones with Electron Microprobe Compositional Mapping," Proceedings of the 12 th International Congress for Electron Microscopy, San Francisco Press, (1990).

Miller, W. R. ; Boettinger, W. J.; Tseng, W. F. ; Pellegrino, J. and Comas, J.; "Controlled Interface Roughness in GaAs/AlAs Superlattices", Mat. Res. Soc. Proc., in press.

Murray, B. T.; Coriell, S. R., McFadden, G. B., "The Effect of Gravity Modulation on solutal Convection during Directional Solidification, J. Crystal Growth 110, 713-723 (1991). 
Murray, B. T.; McFadden, G. B., and Coriell, S. R., "Stabilization of Taylor-Couette Flow due to Time-Periodic Outer Cylinder Oscillation, Phys. Fluids A2, 2147-2156 (1990).

Osella, S. A.; Ridder, S. D.; Biancaniello, F. S.; and Espina, P. I.; "The Intelligent Control of an Inert Gas Atomization Process", JoM, 43, (1991).

Raynes, A. S.; Freiman, S. W.; Gayle, F. W.; and Kaiser, D. L., "Fracture Toughness in $\mathrm{YBa}_{2} \mathrm{Cu}_{3} \mathrm{O}_{5+x}$ Single Crystals: Anisotropy and Twinning Effects," J. Applied Physics, accepted for publication.

Ridder, S. D.; Osella, S. A.; Espina, P. I.; and Biancaniello, F. S., "Advanced Sensors and Process Control for Gas Atomization", submitted for publication in proceedings of the MPIF/APMI conference and exposition, Chicago, (June 9-12, 1991).

Roth R. S.; Hwang, N. M., Rawn, C. J., Burton, B. P., and Ritter, J. J. "Phase equilibria in the systems $\mathrm{CaO}-\mathrm{CuO}$ and $\mathrm{CaO}-\mathrm{Bi}_{2} \mathrm{O}_{3}$ ". Submitted to Journal of the American Ceramic Society 1/15/91.

Sandlin, A. C. and Schaefer, R. J.; "Interfacial Energy Reduction Analysis in Fibrous Structures," submitted to Structure/Property Relationships for Metal/Metal Interfaces, MRS 1991 Spring Meeting.

Sandlin, A. C. and Schaefer, R. J.; "Surface Energy Reduction in Fibrous Monotectic Structures," Metallurgical Transactions, 22A, 1881-1886, (1991).

Sangster, J.; Bale, C. W.; Burton, B. P.; "The System Fe-K"; Journal of Phase Equilibria, V12-1, 46-49. (1991)

Schaefer, R. J.; "Intelligent Processing of Hot Isostatic Pressing," submitted to Proceedings of First Thermal Structures Conference, Charlottsville, VA., Nov 1990.

Schaefer, R. J.; ed.; Hot Isostatic Pressing: Theory and Applications (ASM International, Materials Park, Ohio, 1991).

Schaefer, R. J.; Biancaniello, F. S.; Jiggetts, R. D., "Preparation of Bulk Samples of Copper-Tin Intermetallic Compounds", Submitted for The Metal Science of Joining, 1991.

Schaefer, R. J.; and Janowski, G. M.; "Phase Transformation Effects during HIP of TiAl", Acta Metall. Mater., submitted.

Swartzendruber, L. J.; Kaiser, D. L.; Gayle, F. H.; Bennett, L. H. ; and Roytburd, A; "Low Field Flux Pinning in Twinned and Detwinned Single Crystals of $\mathrm{YB}_{2} \mathrm{Cu}_{3} \mathrm{O}_{6+z^{\prime \prime}}$, Applied Physics Letters 58, 1991, 1566-1568. 
Torardi, C. C.; Pise, J. B.; Santoro, A.; Rawn, C. J.; Roth, R. S.; and Burton, B. P.; " $\mathrm{Sr}_{2} \mathrm{Bi}_{2} \mathrm{O}_{5}$ : A Structure Containing Only 3-Coordinated Bismuth". Accepted, J. Solid State Chem. V93.

Vaudin, M. D.; Blendell, J. E.; and Handwerker, C. A., "DiffusionInduced Grain Boundary Migration in Ceramics", Proceedings of the Symposium on "Structure/Property Relationships for Interfaces", in press.

Wadley, H. N. G. ; Schaefer, R. J. ; Kahn, A. H. ; Ashby, M. F. ; Clough, R. B., Geffen, Y; and Wlassich, J. J.; "Sensing and Modeling of the Hot Isostatic Pressing of Copper Powder", Acta Metall. Mater. 39, 979-986 (1991).

Wheeler, A. A.; McFadden, G. B., Murray, B. T.; and Coriell, S. R., "Convective Stability in the Rayleigh-Benard and Directional Solidification Problems: High Frequency Gravity Modulation, Phys. Fluids A,", in press.

Wheeler, A. A.; Boettinger, W. J.; "Towards a Phase Field Model for Phase Transitions in Binary Alloys", Workshop on Evolution of Phase Boundaries - Institute for Mathematics and its Applications, University of Minnesota, Springer-Verlag, in press.

Wheeler, A. A.; Boettinger, W. J. and McFadden, G. B. "A Phase Field Model for Isothermal Phase Transitions in Binary Alloys", Phys. Rev. A, submitted.

\section{Metallurgical Sensing \& Modeling Group}

Clough R. B; "Application of Acoustic Emission to Studying Fiber Fragmentation as a Process in Single Fiber Composites", to be published in Review of Progress in Quantitative Nondestructive Evaluation, D. Thompson, editor, (1991).

Clough, R. B.; and Schaefer, R. J.; "The Micromechanics of Densification and Distortion", , Fourth International Conf. on Isostatic Pressing, (ISO4), Stratford-Upon-Avon, UK, (1990).

Clough, R. B.; and Schaefer, R. J.; "Densification and Distortion in Isostatic Pressing", submitted to Acta Met. Vol. (1991).

Feldman, A.; Frederikse, H. P. R.; and Norton, S. J.; "Analysis of Thermal Wave Propagation in Diamond Films, SPIE Proceedings, Vol. 1325; Diamond Optics III, 1990, A. Feldman, ed.

Gvishi, M.; Kahn, A. H.; and Mester, M. L.; "Eddy Current Testing of Carbon-Carbon Composites," to be published in the Proceedings. 
Johnson, W. ; Kahn, A. H. ; Chiang, C. K. ; and Wadley, H. N. G. ; "Eddy Current Sensing of Oxygen Content in High- $T_{c}$ superconductors." Review of Progress in Quantitative Nondestructive Evaluation, Vol. 9B, 2085 (1990).

Hwang, J. S.; Lucey, G.; Clough, R. B. ; and Marshall, J.; "Futuristic Solders- Utopia or Ultimate?", to be published in Surface Mount Technology, circa Sept., 1991.

Johnson, W. L.; and Granato, A. V.; "Ultrasonic Relaxation of Interstitial Aluminum in Irradiated Silicon," Phys. Rev. B, $\underline{43}, 9728$ (1991).

Johnson, W. L.; Norton, S. J.; Bendec, F.; and Pless, R.; "Ultrasonic Spectroscopy of Metallic Spheres Using Electromagnetic-Acoustic Transduction," submitted to the Journal of the Acoustical Society of America .

Kahn, A. H. ; Mester, M. L.; and Wadley, H. N. G.; "Eddy Current Techniques for Sample Dimension Measurements During Hot Isostatic Pressing", Proceedings of the Second International Conference on Hot Isostatic Pressing (1989).

Kahn, A. H.; Mester, M. L.; "Through-Transmission Impedance Measurements on Moving Metallic Sheets," to be published in the Proceedings.

Manor, A.; and Clough R.; "In-Situ Determination of Fiber Strength and Segment Length in Composites Using Acoustic Emission", accepted for publication in Composites Science and Technology (1991).

Norton, S. J.; Kahn, A. H.; and Mester, M. L.; "Reconstructing Electrical Conductivity Profiles from Variable-Frequency Eddy Current Mueasurements." Research in NDE, Vol. 1, No. 3, (1989).

Norton, S. J.; Kahn, A. H.; Mauer, F. A.; and Wadley, H. N. G.; "Inverse Problems in the Sensing of Materials Processing," Proceedins of the Intelligent Processing of Materials Conference, 1989, H. N. G. Wadley, ed.

Norton, S. J.; "Reconstructing Stratified Fluid Flow from Reciprocal Scattering Measurements," J. Acoust. Soc. Amer. Vol. $\underline{89}$; June 1990.

Norton, S. J.; Kahn, A. H.; and Mester, M. L.; "Reconstructing Electrical Conductivity Profiles from Variable Frequency Eddy Current Measurements." Research and Nondestructive Evaluation, in press, (1990).

Simmons, J. A.; Drescher-Krasicka, E. ; Wadley, H. N. G.; Rosen, M. ; and Hsieh, T. M. "Ultrasonic Methods for Characterizing the Interface in Composites", Review of Progress in Quantitative Nondestructive Evaluation, Vol. I, 1988. 
Wadley, H. N. G.; Simmons, J. A.; and Drescher-Krasicka, E.; "Ultrasonic Propagation at Cylindrical Metal-Ceramic Interfaces in Composites." Proceedings Materials Research Society Symposium on High Temperature Composites, 1988.

Wadley, H.; Schaefer, R. J.; Clough, R. B.; Kahn, A. H.; Ashby, F.; Geffen, Y.; and Wlassich, J. J.; "Modeling of Hot Isostatic Pressing of Copper Powders", Acta Met. (1991). 


\section{INDUSTRIAL AND ACADEMIC INTERACTIONS}

The research programs of the Metallurgy Division are designed and carried out in support of industrial and scientific needs. Specialized facilities within the Division, including metals processing and nondestructive evaluation, attract scientists from both academic and industrial organizations for cooperative research efforts. Interactions with industry, universities, and professional organizations are viewed as an important element of our work with collaborative programs, consulting and general involvement with outside groups being a long standing practice.

In 1991, the Division performed collaborative research with many private organizations through its Research Associate and Guest Scientist programs and other arrangements. Representative examples of such interactions include:

\section{INDUSTRY}

1. American Ceramic Society (ACerS)

Dr. Peter Schenck is collaborating with ACerS and the industrial sponsors of the NIST-ACerS phase diagram optimization program, in the development of a graphical phase diagram database for ceramic and other inorganic systems.

2. American Electroplaters and Surface Finishers Society (AESF)

C. E. Johnson collaborated with Dr. Fred Ancher of Union Carbide to establish two Technology Transfer and Educational Sessions on Electrocomposites. The sessions, organized by AESF, brought together participants from industry, government, and universities interested in metal matrix electrocomposites.

3. Aluminum Association

R. J. Fields is working with a group of company members of the Aluminum Association to identify and eliminate technical impediments to the commercialization of aluminum-matrix composites.

4. Aluminum Association

The Aluminum Association represents many U.S. and Canadian companies. The Association has contributed financial support in the form of a Cooperative Research Associate and through its contract with DMC. Member companies have supplied materials and have invited NIST researchers to their operating facilities for testing. Efforts to commercialize the sensor and broaden applicability are continuing. 
5. Applied Technology Enterprises and Carnegie Mellon Research Institute

HIP densification of high-performance iron-based rare earth permanent magnet materials is being investigated in a collaborative effort between NIST (R. J. Schaefer), CMRI and Applied Technology Enterprises, Ltd.

6. ASM International

The ASM/NIST Data Program for Alloy Phase Diagrams was completed with the publication of the 2nd edition of "Binary Alloy Phase Diagrams". This 3-volume edition contains nearly 3000 binary systems, of which 2500 were assessed by the editors associated with the Program. It represents the most complete and up-to date reference on binary phase diagrams and associated data. (ASM/NIST Data Program staff at NIST: L. H. Schwartz, E. N. Pugh, J. Smith, U. R. Kattner and B. P. Burton).

7. BDM International

A collaborative effort on the development of intelligent control for hot isostatic pressing is jointly funded by DARPA and NIST. Scientists from the Metallurgical Processing, Metallurgical Sensing and Modelling, and Mechanical Properties and Performance Groups worked with BDM International to develop a process model and control strategy for HIP densification of advanced alloys.

\section{Collaborative Testing Services}

T. R. Shives and D. E. Harne participated again this year in four roundrobin test programs designed to measure proficiency in tensile, hardness, and fastener testing.

9. Commonwealth Aluminum

T. R. Shives and R. J. Fields are studying aluminum-matrix composite materials donated by Commonwealth Aluminum to better understand how spherical alumina particles can improve the properties of aluminum alloys.

10. Crucible Compaction Metals

The densification and distortion of titanium powders subjected to hydrostatic and deviatoric stress states have been evaluated in a collaborative effort by NIST (R. Fields and R. Schaefer) and Crucible Compaction Metals. This information is to be used in finite element modelling of shape changes during hot isostatic pressing. 
11. Crucible Materials Corp., General Electric Co., Martin Marietta Energy Systems, and Pratt \& Whitney Aircraft.

The NIST-industrial consortium on automated processing of rapidly solidified metal powders by high pressure inert gas atomization, formed in FY 1988, has begun a second three year study. The NIST supersonic inert gas metal atomizer (SiGMA) in the Metallurgy Division's metals processing laboratory has been the focal point of this pioneering work in advanced sensor development and Artificial Intelligence (AI) control. Scientists from the participating companies, in collaboration with NIST scientists, have developed an in-situ particle size measurement sensor and integrated this device with an AI automatic control system.

12. DuPont (Wilmington, DE)

Dr. John Hastie is collaborating with Drs. R. Gonzalez, U. Klabunde and others of DuPont on the development of process mechanisms for titanium extractive metallurgy.

13. EG\&G Rocky Flats

Members of the Corrosion Group (U. Bertocci) are assisting personnel of EG\&G Rocky Flats with problems related to corrosion associated with low level nuclear waste disposal.

14. E. I. DuPont de Neumours

B. P. Burton has been collaborating with C. C. Torardi and J. B. Parise on the structural characterization of phases in the Sr-Bi-Ca$\mathrm{Cu}-\mathrm{O}$ oxide high- $\mathrm{T}_{\mathrm{c}}$ superconductor system.

15. EPRI (Electric Power Research Institute)

EPRI continues to fund a multi-year program within the Corrosion Data Center (D. B. Anderson) to develop computer programs to guide electric utility materials and design engineering personnel in materials selection and operating controls to minimize critical equipment failures. The program focuses on selected applications in power plant condensers, steam generators, flue gas desulfurization systems and service waters and is supported by two Research Associates assigned by the National Association of Corrosion Engineers (NACE).

16. Exxon Research, Corporate Headquarters

Roger Clough, of the Metallurgical Sensing and Modeling Group, is collaborating with SRI in Princeton, NJ, in helping them acoustic emission to locate and enumerate fiber fractures in fiber and discontinuous fiber/polymer matrix composites in order to model damage due to deformation. More collaborative efforts are being planned for this coming year. 


\section{Fluoramics Corporation}

The Magnetic Materials Group is cooperating with Fluoramics in their attempt to make flexible magnetic bearing material using high temperature superconductors. Flux pinning and magnetic levitation in material prepared by fluoride flux methods is under study. Results are being used by Fluouramics to help improve their production process.

18. General Dynamics Corp.

F. W. Gayle is collaborating with General Dynamics (E.S. Balmuth) in the microstructural analysis of new, low density aluminum alloys for heavy gage plate aerospace applications.

19. General Electric

A collaborative effort among Grant Rowe (GECRD); B. Mozer (Reactor Radiation Division) and the Metallurgy Division led to the determination of the crystal structure of the orthorhombic intermetallic compound $\mathrm{Ti}_{2} \mathrm{AlNb}$.

20. General Motors

(a) Members of the Division (L. J. Swartzendruber) have consulted with General Motors and other members of the Division on a NDE project to measure case hardening depth in steel axels.

(b) General Motors Research Laboratories (GMR) will provide samples for the Magnetic Materials Group to measure magnetization over a wide temperature range and make magnetocaloric effect calculations using this data.

21. GMW Associates

The Magnetic Materials Group and GMW Associates will cooperate in the testing of iron core magnets of special design. These magnets are designed to allow very rapid changes in the applied magnetic field when measuring materials using equipment such as vibrating sample magnetometers.

22. Harry Diamond Laboratory

In cooperation with $\mathrm{Dr}$. Brody at Harry Diamond Laboratories, Dr. Peter Schenck has laser deposited lead zirconate titanate (PZT) thin films on silicon and is involved in spectroscopic characterization of the deposition vapor. PZT is a prime candidate for use in ferroelectric thinfilm non-volatile memory chips. 
23. IBM

The Electrodeposition Group in cooperation with the Magnetic Materials Group has for the past 4 years maintained a CRDA with IBM, Yorktown Heights) to study the synthesis of artificial superlattices and to measure their magnetic properties (D. Lashmore, L. Bennett, C. R. Beauchamp and E. Soltani).

24. Inland Steel

Drs. Plante and Bonnell have been collaborating with Dr. H. Pielet of Inland on the thermochemistry of blast furnace and steel slags and inorganic inclusions in steel. Dr. Pielet provides slag samples and data from plant experience, Dr. Plante is providing thermochemical data from high temperature mass spectrometry, while Dr. Bonnell supplies database and model techniques.

25. Intersonics, Inc.

Dr. Bonnell consults with Dr. S. Krishnan regarding levitation of liquid metals, pyrometric and emissivity measurements at high temperatures, and related studies.

26. J. Stefan Institute, Ijubljana

The Corrosion Group (U. Bertocci) is collaborating with Dr. C. Zevnik of the Stefan Institute on a NIST-Yugoslavia Joint Project on monitoring corrosion in heat exchangers for electrical power stations.

27. Liberty Technologies

R. J. Fields and T. R. Shives measured the elastic properties of two stainless steel samples furnished by the company. These materials are intended for use in a sensitive nuclear power plant application requiring precise knowledge of these properties.

28. $3 M$ and $G E$

Technology transfer seminars on electrochemically produced metal matrix composites were held with engineers and project managers of $3 \mathrm{M}$ and $\mathrm{GE}$ and members of the Electrodeposition Group. The seminars provided a media to present an alternative process to develop continuous fiber reinforced metal matrix composites. (C. E. Johnson, and D. S. Lashmore)

29. Martin Marietta Laboratories (Baltimore), Martin-Marietta Astronautics Group (Denver)

Martin-Marietta Laboratories (J. R. Pickens and F. H. Heubaum), MartinMarietta Astronautics (W. T. Tack) and NIST (F. W. Gayle) are collaborating in a study of the physical metallurgy and microstructure of a new class of weldable, ultra-high strength aluminum alloys. 
30. Martin Marietta Astronautics Group (Denver)

F. W. Gayle is collaborating with Martin Marietta Astronautics (W. T. Tack) in the development of thin wall tubing of Al-Cu-Li alloys for heat exchangers for the National Aerospace Plane (NASP).

31. Materials Innovation

The electrodeposition group has just established a CRDA with MI to investigate the electrochemical synthesis of particulate composite materials (D. R. Kelley and D. S. Lashmore).

32. Materials Technology Institute of the Chemical Process Industries (MTI)

MTI continues to support a multi-year program in the Corrosion Data Center (D. B. Anderson) to develop expert systems for selection of materials for storage and handling of hazardous chemicals. Systems are based on knowledge rules defined during discussions with consulting experts representing a broad range of industrial experience. MTI, through the National Association of Corrosion Engineers (NACE) supports two Research Associates in the Corrosion Data Center to aid in this program.

33. Microelectronic and Computer Technology Corporation (MCC)

A collaborative program is underway between MCC (Dr. Colin Mackay) and NIST (Dr. Carol Handwerker) to identify the critical variables controlling the solderability of electronic components after longterm storage.

34. National Association of Corrosion Engineers (NACE)

NACE continues to support the Corrosion Data Center (D. B. Anderson) through a joint agreement with NIST. NACE provides full time Research Associates at NIST plus considerable staff support cost sharing for programs to collect, evaluate and disseminate corrosion data for engineering materials.

35. NASA/Cal Tech Jet Propulsion Lab (Pasadena, CA)

Dr. Bonnell collaborates and consulates on design of levitation systems for space applications.

36. Naval Research Laboratory

C. A. Handwerker has been collaborating with Dr. Steven Hues of NRL on the oxidation of $\mathrm{Cu}-\mathrm{Sn}$ intermetallic compounds. Dr. Hues performs scanning Auger electron spectroscopy experiments on the oxidation of bulk $\mathrm{Cu}-\mathrm{Sn}$ intermetallic materials prepared at NIST by by rapid solidification and HIP' ing. 
37. Naval Research Laboratory

B. P. Burton is collaborating with J. E. Osburn and M. Mehl of the Naval Research Laboratory on first principles phase diagram calculations.

38. Rocketdyne

Members of the Electrodeposition Group are aiding engineers at Rocketdyne in the electroforming of rocket thruster engines. Investigations at NIST on problems involving soft nickel electroforms are of primary interest (D. S. Lashmore, C. E. Johnson and D. R. Kelley).

39. Sandia National Laboratories

(Department of Energy)

Sandia National Laboratories (F. Yost) and NIST (W. J. Boettinger and C. A. Handwerker) are collaborating on a study of the effects of oxidation on the solderability of $\mathrm{Cu}-\mathrm{Sn}$ intermetallic compounds both with and without fluxes. The ability of a component to be soldered without flux eliminates the need for CFC's in soldering, a national environmental goal.

40. Solution Model Database

Groups currently using or evaluating the IMCC model include Dr. Howard Pielet, Inland Steel; Prof. K. S. Spear, Penn State University; Dr. Chad Sheckler, Alfred University; Prof. Paul Davis, Brigham Young University; Prof. Steve Benson, Univ. North Dakota; Prof. Tom Roberts, Milwaukee Area Technical College; Dr. C. David Rogers, Carnegie-Mellon Institute and USS of USX; Prof. Arthur E. Morris, University of Missouri-Rolla, Dr. Shankar Krishnan, Intersonics, Inc., Dr. Mark Huntelaar (Dr. Patrick O'Hare), Robert Wilson (Dr. Dennis Bickford), Westinghouse-Savannah River Laboratories.

41. SRI (Stanford Research International)

Drs. Hastie and Bonnell are collaborating with Dr. D. Hildenbrand of SRI in the mass spectral analysis of complex high temperature vapors. NIST and SRI are using techniques which are unique to each laboratory but are nevertheless complementary.

42. Stanford Research Institute

Roger Clough, of the Metallurgical Sensing and Modeling Group, is collaborating with SRI in Princeton, NJ, in helping them duplicate his method for using acoustic emission to locate fiber fractures in single fiber metal matrix composites. Initial results at SRI, made with SiC fibers and a nickel superalloy matrix, have been successful. 
43. Sundstrand Corporation

R. J. Fields collaborated with Messrs. K. Peters, B. Markekett, and J. Readman to determine the cause of failure in two advanced boiler systems. Fields' advice on the suitability of Type 316L stainless steel for this application was sought as a result of his publication record in this area.

44. USX/Carnegie-Mellon

Dr. Rogers of Carnegie-Mellon Institute (formerly U.S. Steel of USX) is developing process models for melt shop use. Dr. Bonnell is working with Dr. Rogers to develop a form of the NIST Steel Slag Model for use as the chemistry component of such process models.

45. Westinghouse Science and Technology Center

Calculations of the onset of convective and morphological instabilities during solidification of silver bromide-doped lead bromide alloys have been carried out in collaboration with Dr. N. B. Singh. Crystal growth experiments in space are planned in order to prepare homogeneous crystals for use in acousto-optic and optoelectronic devices. 


\section{INDUSTRY/UNIVERSITY}

1. BHABHA Atomic Research Centre (Government of India) University of Poona

A cooperative project is underway with the BHABHA Atomic Research Center (Dr. C. K. Gupta) and the University of Poona (Dr. A. P. B. Sinha). This project is part of the Indo-US Physical, Materials and Marine Sciences Collaboration Program and the objective of this project is to study the influence of nitrogen content on the stress corrosion cracking behavior of stainless steels (alloy 316L). NIST Corrosion Group (U. Bertocci) has provided material for this study and complimentary experiments will be conducted at the various institutions. A workshop on the Corrosion Science and Technology on Stainless Steels is being planned to be held in India.

2. Beijing University of Science and Technology (BUST) (Government of China)

The United Nations Industrial Development Organization (UNIDO) funded a six-month fellowship for Zuyu Qu, a Guest Scientist from BUST, to work in the Corrosion Data Center (D. B. Anderson) to develop an atmospheric corrosion database in for the Beijing Corrosion and Protection Center.

3. Composites Technology Transfer Workshop

D. S. Lashmore presented an overview of the development of electrochemically produced metal matrix composite precursors. The workshop, organized by West Virginia University, brought together participants from Boeing, Pratt Whitney, 3M, NASA, LANL, NRL and NIST interested in the development and production of metal matrix composites (D. S. Lashmore, C. E. Johnson and D. R. Kelley).

4. Department of Treasury

Bureau of Engraving and Printing (BEP)

The Electrodeposition Group has completed its collaboration with BEP to develop wear resistant coatings for wiper blades used on the water-wipe currency printing presses. (C. E. Johnson, D. R. Kelly, J. Mullen and D. S. Lashmore).

\section{NIST Metals Processing Laboratory}

Facilities in this laboratory are available for preparation of special samples for various materials characterization studies. University and industry scientists can assist in sample processing for independent or collaborative research projects involving alloy development, rapid solidification and particulate consolidation. During the past year, investigators from Crucible Materials Corp., General Electric Co., INCO, CMS Metals, NASA, DOE, Bureau of Mines, DTRC, Northwestern Univ, and Univ. of Wisconsin have interacted in this program. 


\section{UNIVERSITIES}

1. ACCESS e. V., Aachen, Germany

Calculations of convection and interface stability during the directional solidification of succinonitrile-acetone alloys are being carried out in conjunction with ground and space experiments on this transparent alloy planned by $\mathrm{K}$. Leonartz.

2. Boris Kidric Institute (BKI), Belgrade, Yugoslavia

A collaborative activity is underway between $\mathrm{Dr}$. Hastie and $\mathrm{Dr}$. Zmbov of BKI for the mass spectrometric analysis of complex high temperature processes, including plasma deposition of amorphous silicon.

3. Cambridge University

Predictive models for the hot isostatic pressing of particulate composites are being developed by Prof. M. F. Ashby and graduate students at Cambridge University in collaboration with Metallurgy Division scientists.

4. Centre d'Etudes Nucleaires de Grenoble

Theoretical investigations of cellular growth during direction solidification of tin alloys containing bismuth are being carried out in conjunction with experiments to determine interface temperature using Seebeck measurements by J. J. Favier and collaborators.

5. Chonnam University

A collaborative effort is underway between C. A. Handwerker of NIST and Prof. D. J. Lee of Chonnam University, Kwangju, Korea to study interface properties of Al-based metal matrix composites under an NSF-KOSEF joint program.

6. Free University of Brussels

Drs. Hastie and Bonnell are collaborating with Dr. J. Drowart (Brussels) on a survey of ionization cross section usage in high temperature mass spectrometry.

7. George Washington University

W. J. Boettinger serves as a Professional Lecturer in Engineering Science at George Washington University, where he teaches the graduate course "Transformations in Materials". 
8. Indira Gandhi Institute (IGI) Kalpakkam, India

A collaborative activity is underway between Dr. Hastie and Dr. Mathews of IGI for the mass spectrometric investigation of materials at very high temperatures generated by laser heating.

9. Institute of Solid State Physics, USSR Academy of Sciences

A cooperative program with Prof. Dr. V. I. Nikitenko of the Soviet Academy of Sciences and F. W. Gayle and D. Kaiser of NIST is underway to examine the dynamics of twin boundary migration in high temperature superconductors

10. Institute of Solid State Physics, Institute for Microstructural Study, KFA, Julich, Germany

Collaborative research on the atomic structure of defects and interfaces in Ti-Al-Nb intermetallic phases has been initiated with Prof. K. Urban and Ph.D. student A. Thust. The research is a continuation of a study at NIST on mechanisms, crystallography and thermodynamics of phase transformations in $\mathrm{Ti}-\mathrm{Al}-\mathrm{Nb}$ system.

11. Iowa State University

A collaborative effort is underway between NIST (F. W. Gayle) and ISU (Prof. Alan Goldman) to characterize single crystals of the Al-Cu-Ii quasicrystal by inelastic neutron scattering.

12. Johns Hopkins University

The magnetic properties of detwinned superconducting $\mathrm{YBa}_{2} \mathrm{Cu}_{3} \mathrm{O}_{6+x}$ single crystals fabricated at NIST (D. Kaiser and F. W. Gayle) are being measured at Johns Hopkins (K. Moorjani) using the recently-developed magnetically modulated microwave absorption (MAMMA) technique. The objective of this effort is to study the details of the superconducting transition in twinned and detwinned crystals in order to further the understanding of the mechanism of high temperature superconductivity.

13. Johns Hopkins University

The Metallurgical Sensing and Modeling Group is collaborating with Dr. James Wagner at Johns Hopkins University in the development of interferometric techniques for detecting ultrasound and in the application of laser ultrasonic techniques to real-time characterization of liquid/solid interfaces in directionally solidifying metals.

14. Korea Advanced Institute of Science and Technology

C. A. Handwerker and J. R. Manning are collaborating with Prof. D. $N$. Yoon of KAIST on studies of the effects of diffusion-induced stresses on interface stability. 
15. Metalurski Institut (MI), Ljubljana, Yugoslavia

Dr. Hastie is collaborating with Dr. V. Presern of MI on a NIST-Yugoslavia Joint Project research program in steel inclusion thermochemistry.

16. Rice University

Dr. Bonnell continues to provide consultation support to the High Temperature Group in the Chemistry Department on levitation and thermophysical properties of liquid metals.

17. University of Bristol (Great Britain)

David Lashmore has just received a NATO grant to establish a cooperative program with the University of Bristol to study damping in compositionally modulated alloys.

18. University of Florida

A collaborative effort with Professor Abbaschian (University of Florida) on the in-situ monitoring of crystal growth using the MEPHISTO furnace is underway. Experiments and calculations on bismuth alloys containing tin are planned.

19. University of Grenoble and C.N.R.S.

B. P. Burton is collaborating with Professor A. Pasturel on first principles phase diagram calculations.

20. University of Houston

In this joint effort between the University of Houston (Prof. S. Moss) and NIST (D. Kaiser, F. W. Gayle), detwinned single crystals of $\mathrm{YBa}_{2} \mathrm{Cu}_{3} \mathrm{O}_{6+x}$ are being measured by high-resolution $\mathrm{x}$-ray diffraction techniques at temperatures in the range $10-298 \mathrm{~K}$ to search for a structural transition associated with the onset of superconduc-tivity. Fully untwinned crystals are required for these measurements to eliminate ambiguities introduced by the presence of twin boundaries which are present in all as-grown crystals.

21. University of Maryland

R. deWit and R. J. Fields are collaborating with Professor G. R. Irwin to further the understanding of crack arrest and reinitiation behavior in steel. In particular, the relation between thickness reductions and fracture toughness is being studied. 
22. University of Maryland

A collaborative study between the University of Maryland (Dr. A. Roitburd) and NIST (D. Kaiser, F. W. Gayle, L. Swartzendruber, L. Bennett) involves theoretical aspects of twin boundary migration under an applied stress and flux pinning by twin boundaries in $\mathrm{YBa}_{2} \mathrm{Cu}_{3} \mathrm{O}_{6+\mathrm{x}}$.

23. University of Virginia

A collaborative study was initiated between the Electrodeposition Group and $\mathrm{Dr}$. H. Wadley (UVa) that involves the modeling of hot isostatic pressing parameters for consolidation of electrochemically produced metal matrix composite precursors (D. S. Lashmore, G. R. Stafford, C. E. Johnson, D. R. Kelley and P. N. Sharpless).

24. University of Pennsylvania

R. J. Fields collaborated with Professor N. Aravas in the evaluation of three yield-surface models for metal powders using data developed by $S$. R. Low, III, and Fields at NIST.

25. University of Surrey, U.K.

Collaborate research between Stephen Norton of the Metallurgy Division and Professor John Bowler of the University of Surrey has resulted in a general eddy-current inversion theory for determining 3-D electrical conductivity distributions in metals.

26. University of Washington

A collaborative effort with Professor Lucien Brush on the solidification of alloys is underway. The stability of a spherical particle growing into a highly supercooled melt is being investigated numerically.

27. University of Windsor

The Electrodeposition Group in cooperation with IBM is conducting a joint program on Lorentz Electron Microscopy with the University of Windsor in Canada (D. S. Lashmore, J. L. Mullen and C. R. Beauchamp).

28. University of Wisconsin at Madison

Cooperative programs underway with J. Perepezko and A. Chang of the University of Wisconsin have focussed on studies of microstructural development and phase diagram relationships in the Ti-Al-Nb system. 

Aluminum Association

Task Group on Development of Aluminum Metal-Matrix

Composites

R. J. Fields

American Academy of Mechanics

R. deWit

R. J. Fields

American Association of Crystal Growth

S. R. Coriell, Executive Committee

American Ceramic Society

C. A. Handwerker, Long-Range Planning Committee

American Electroplaters and Surface Finishers Society

C. E. Johnson, Electrocomposites Committee Alloy Deposition Committee Aerospace and Light Metals Committee

D. S. Lashmore, Alloy Deposition Subcommittee

American Institute of Mining, Metallurgical and Petroleum Engineers

The Metallurgical Society

W. J. Boettinger, Solidification Committee

R. E. Ricker, Committee on Corrosion and Environmental Effects

R. J. Schaefer, Solidification Committee

R. D. Shull, The Chemistry and Physics of Materials Committee

R. D. Shul1, The Titanium Committee

American Physical Society

Materials Physics Topical Group

C. A. Handwerker, Member of Steering Committee

ASM INTERNATIONAL

Corrosion and Environmental Effects Committee

R. E. Ricker

Heat Treating Steering Committee

H. T. Yolken

Journal of Phase Equilibria, Editorial Committee

F. W. Gayle

Residual Stress Committee

L. Mordfin 
Technology Transfer Committee

E. N. Pugh

Washington, DC Chapter - Executive Committee

R. E. Ricker, Vice Chairman

ASTM

Standing Committee on Publications

L. Mordfin, Vice Chairman

B2: Nonferrous Metals and Alloys

S. D. Ridder

B5: $\quad$ Copper and Copper Alloys

L. J. Swartzendruber

B7: $\quad$ Light Metals

R. D. Shull

W. J. Boettinger

B8: $\quad$ Metallic and Inorganic Coatings

B8.10: General Test Methods

C. E. Johnson

D. S. Lashmore

B8.10.03: Microhardness Testing

C. E. Johnson, Liaison to EO4

D. S. Lashmore, Liaison to EO4

B9: Metal Powders and Metal Powder Products

J. R. Manning

E4: Metallography

E4.05: Microhardness

C. E. Johnson

D. S. Lashmore

E4.14: Quantitative Metallography

R. J. Fields

E7: $\quad$ Nondestructive Testing

L. Mordfin, Executive Subcommittee

L. J. Swartzendruber

L. H. Bennett

E7:04: Acoustic Emission

J. A. Simmons

R. B. Clough

E7.10:04: Infrared NDT Methods

L. Mordfin, Chairman 


\begin{tabular}{|c|c|}
\hline E7.91: & $\begin{array}{l}\text { USA Committee for ISO TC } 135 \\
\text { L. Mordfin, Chairman }\end{array}$ \\
\hline E9: & $\begin{array}{l}\text { Fatigue } \\
\text { R. dewit } \\
\text { R. J. Fields }\end{array}$ \\
\hline E24: & $\begin{array}{l}\text { Fracture Testing } \\
\text { R. E. Ricker } \\
\text { R. deWit } \\
\text { R. J. Fields } \\
\text { S. R. Low, III }\end{array}$ \\
\hline E24:02 & $\begin{array}{l}\text { Fractography and Associated } \\
\text { Microstructures } \\
\text { G. E. Hicho }\end{array}$ \\
\hline E24.04: & $\begin{array}{l}\text { Environmental Cracking } \\
\text { R. E. Ricker }\end{array}$ \\
\hline E24.06: & $\begin{array}{l}\text { Fracture Mechanics Applications } \\
\text { J. H. Smith } \\
\text { G. E. Hicho }\end{array}$ \\
\hline E28: & $\begin{array}{l}\text { Mechanical Testing } \\
\text { L. Mordfin, Executive Subcommittee }\end{array}$ \\
\hline E28.06 & $\begin{array}{l}\text { Hardness } \\
\text { T. R. Shives } \\
\text { J. H. Smith }\end{array}$ \\
\hline E28.06.07: & $\begin{array}{l}\text { Hardness Test Block Intercomparison } \\
\text { T. R. Shives } \\
\text { R. J. Fields }\end{array}$ \\
\hline E28.13: & $\begin{array}{l}\text { Residual Stress Measurement } \\
\text { L. Mordfin, Chairman }\end{array}$ \\
\hline E49: & $\begin{array}{l}\text { Computerization of Material Property } \\
\text { Data } \\
\text { D. B. Anderson, Chairman }\end{array}$ \\
\hline E49.02: & $\begin{array}{l}\text { Corrosion Data Formats } \\
\text { D. B. Anderson, Chairman }\end{array}$ \\
\hline E49:91: & $\begin{array}{l}\text { D. B. Anderson, Awards Committee } \\
\text { Chairman }\end{array}$ \\
\hline G1: & $\begin{array}{l}\text { Corrosion of Metals } \\
\text { D. B. Anderson } \\
\text { M. R. Stoudt }\end{array}$ \\
\hline
\end{tabular}



G1.03.1: Corrosion Data Formats Task Group D. B. Anderson, Chairman
G1.06: SCC and Corrosion Fatigue
R. E. Ricker
G1.10: Corrosion of Metals in Soil
E. Escalante
G1.10.01: Measurement of pH of Soil
E. Escalante, Task Group Leader
G1.10.02: Measurement of Soil Resistivity
E. Escalante
G1.11: Electrochemical Measurements in Corrosion
R. E. Ricker
G1.14: Corrosion of Steel in Concrete
E. Escalante

Electrochemical Society

Corrosion Division

R. E. Ricker

Electronics Division

R. E. Ricker

Symposia Subcommittee

D. S. Lashmore

Gordon Conference, Physical Metallurgy Conference for Summer 1991

W. J. Boettinger, Co-Chairman

High Temperature Science Journal

J. W. Hastie, Editorial Board Member

International Advisory Committee and Program Committee for International Conference on Rapidly Quenched Metals

W. J. Boettinger, Member

International Organizing Committee for Interational Workshop on Magnetic Properties of Fine Particles and their Relevance to Materials Science

R. D. Shull, Member

International Standards Organization

TC58.SC-3: Cylinder Design

J. H. Smith, Delegate 
TC107:

Metallic and Other Non-Organic Coatings

C. E. Johnson, Delegate

TC107.02: Methods of Inspection and Co-Ordination of Test Methods

C. E. Johnson, Delegate

TC107.03: Electrodeposited Coatings and Related Finishes

C. E. Johnson, Delegate

TC135: Nondestructive Testing

L. Mordfin, Delegate

International Conference Series on High Temperature

Materials Chemistry, Organizing Committee

J. W. Hastie, Member

International Union of Pure and Applied Chemistry

Commission II- 3

High Temperature and Solid State Chemistry

J. W. Hastie, Titular Member

Journal of the American Ceramic Society

C. A. Handwerker, Associate Editor

Materials Research Society

R. E. Ricker

E. Escalante

A. C. Fraker

Materials Science and Engineering

L. H. Bennett, Associate Editor

National Aeronautics and Space Administration

High Temperature Advisory Committee

D. W. Bonnell, Member

National Association of Corrosion Engineers (NACE)

T. 3

Corrosion Science and Technology

Committee

T.3E: Unit Committee on SCC and Corrosion

Fatigue

R. Ricker

T.3K: Corrosion and Other Deterioration Phenomenon Associated with Concrete

E. Escalante

T.3L: Unit Committee on Electrochemical

Techniques

R. Ricker 
T.3U: Computers in Corrosion Control

D. B. Anderson

T.3U.1: Task Group on Corrosion Data Formats

D. B. Anderson, Chairman

NACE Research Committee

E. N. Pugh, Past Chairman and Chairman of Research in Progress Symposium Sub-Committee

NACE-NIST Corrosion Data Program

Joint Coordination Committee

R. E. Ricker

D. B. Anderson

NACE Baltimore-Washington Chapter Executive Committee

R. Ricker, Treasurer

National Research Council

National Materials Advisory Board

Committee on Industrial Energy Utilization

H. T. Yolken

National Research Council

Transportation Research Board

E. Escalante, Panel Member

Office of Energy-Related Inventions, National Institute of Standards and Technology

L. H. Bennett, Reviewer of Invention Disclosures

Research in Nondestructive Evaluation, A Journal of the American Society for Nondestructive Testing

H. T. Yolken, Editor-In-Chief

Sigma Xi

R. deWit

Society of Automotive Engineers/ASTM

Unified Numbering System for Metals and Alloys

L. H. Bennett, NIST Representative

Third International Conference on Hot Isostatic Pressing

R. J. Schaefer, Program Committee

U.S. Department of Transportation

Technical Hazardous Liquid Pipeline Safety Standards

Committee

R. J. Fields, Secretary

Washington Academy of Sciences

R. dewit 
White House, Office of Science and Technology Policy, Council on Materials, Working Group on Materials

Processing and Synthesis

H. T. Yolken, Chairman 



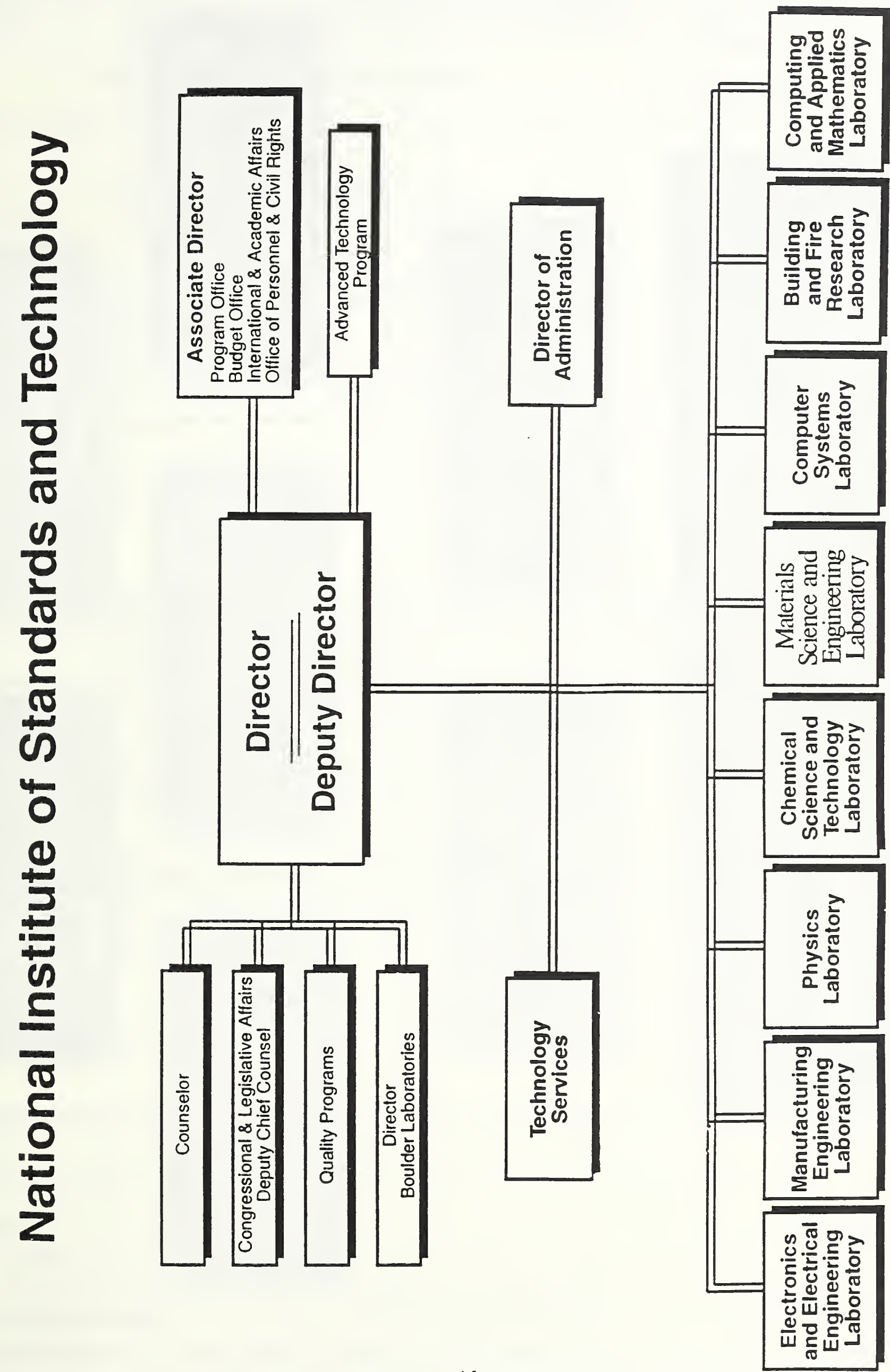




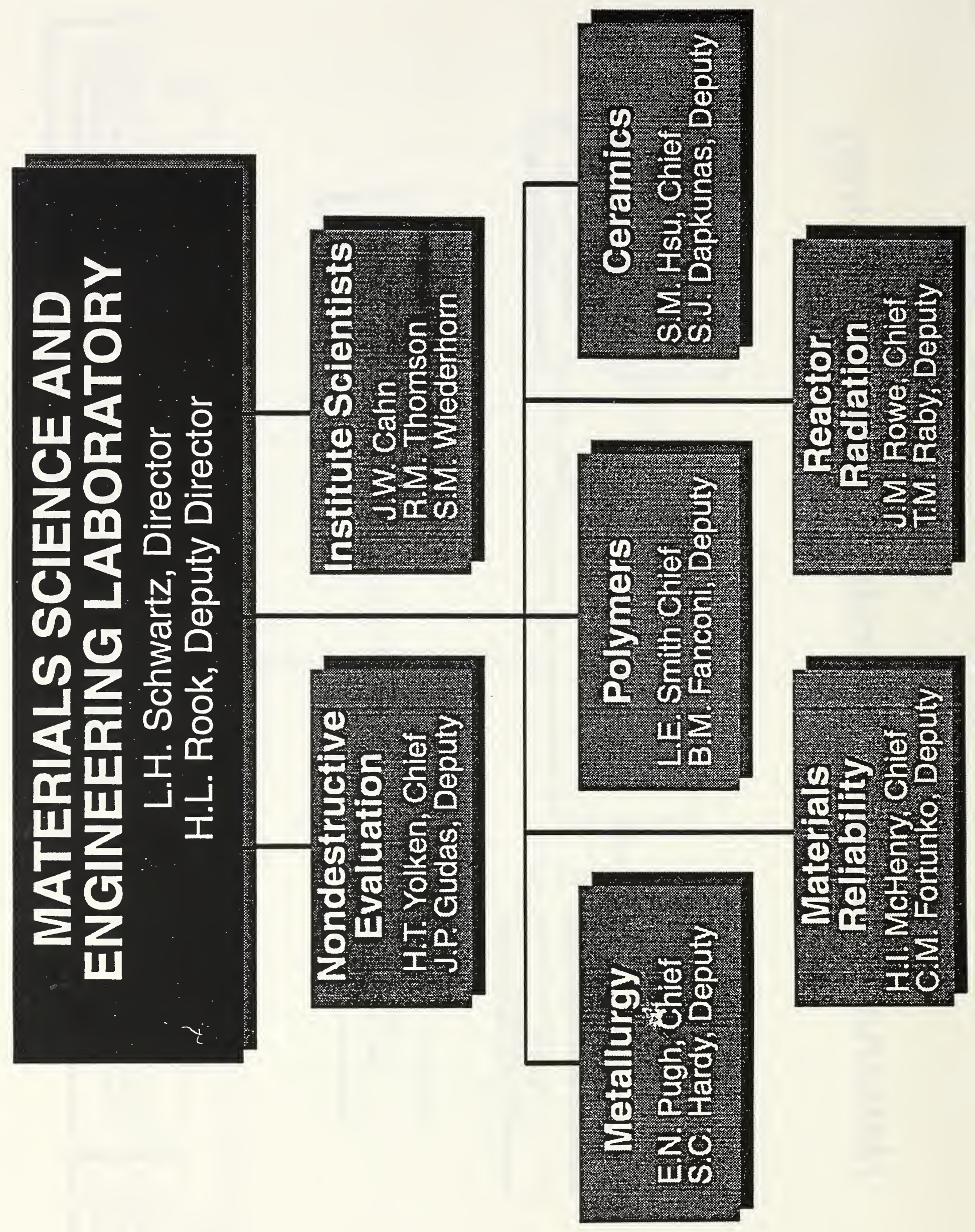


1. PUBLCATION OR REPORT NUMBER

(REV. 3-89)

\section{BIBLIOGRAPHIC DATA SHEET}

3. PUBLCATION DATE

December 1991

\section{TITLE AND SUBTITLE}

Materials Science and Engineering Laboratory Metallurgy Division, Technical Activities 1991

5. AUTHOR(S)

E. Neville Pugh and Stephen C. Hardy

\begin{tabular}{l|l|}
\hline 6. PERFORMING ORGANIZATION (IF JOINT OR OTHER THAN NIST, SEE INSTRUCTIONS) & CONTRACT/GRANT NUMBER \\
$\begin{array}{l}\text { U.S. DEPARTMENT OF COMMERCE } \\
\text { MATIONAL INSTITUTE OF STANDARDS AND TECHMOLOGY } \\
\text { GATTHERSBURG, MD 20899 }\end{array}$ & $\begin{array}{c}\text { 8. TYPE OF REPORT AND PERIOD COVERED } \\
10 / 1 / 90 \text { to } 9 / 30 / 91\end{array}$ \\
\hline
\end{tabular}

9. SPONSORIMG ORGANIZATION NAME AND COMPLETE ADDRESS (STREET, CITY, STATE, ZIP) UTERATURE SURVEY, MENTION IT HERE.)

This report summarizes the FY 1991 activities of the Metallurgy Division of the National Institute of Standards and Technology (NIST). These activities center upon the structure-processing-properties relations of metals and alloys and on methods of measurement; and also include the generation and evaluation of critical materials data. Efforts comprise studies of metals processing and process sensors; advanced materials, including metal matrix composites, intermetallic alloys and superconductors; corrosion and electrodeposition; mechanical properties; magnetic materials; and high temperature reactions

The work described also includes two cooperative programs with professional societies (the Alloy Phase Diagram Program with ASM International, and the Corrosion Data Program with the National Association of Corrosion Engineers); two with trade associations (the Temperature Sensor Program with the Aluminum Association, and the Steel Sensor Program with the American Iron and Steel Institute); and several with industry including the Powder Atomization Consortium with three companies.

The scientific publications, committee participation, and other professional interactions of the 74 full-time and part-time permanent members of the Metallurgy Division and its 46 guest scientists are identified.

12. KEY WORDS (6 TO 12 ENTRIES; ALPHABETICAL ORDER; CAPITAUZE ONLY PROPER MAMES; AND SEPARATE KEY WORDS BY SEMICOLONS)

Corrosion; Electrodeposition; Magnetic Properties; Metals Processing; Metallurgy; Process Sensors

13. AVAILABIUTY

UNUMITED

FOR OFFICIAL DISTRIBUTION. DO NOT RELEASE TO NATIONAL TECHNICAL INFORMATION SERVICE (NTIS).

ORDER FROM SUPERINTENDENT OF DOCUMENTS, U.S. GOVERNMENT PRINTIMG OFFICE, WASHINGTON, DC 20402.

ORDER FROM NATIONAL TECHNICAL INFORMATION SERVICE (NTIS), SPRINGFIELD, VA 22161.
14. MUMBER OF PRINTED PAGES 136

15. PRICE A07 


